\author{
SANDIA REPORT \\ SAND2006-6763 \\ Unlimited Release \\ Printed November 2006
}

\title{
Experimental Characterization of Spin Motor Nozzle Flow
}

Carl W. Peterson, John F. Henfling, Rocky J. Erven

Prepared by

Sandia National Laboratories

Albuquerque, New Mexico 87185 and Livermore, California 94550

Sandia is a multiprogram laboratory operated by Sandia Corporation,

a Lockheed Martin Company, for the United States Department of Energy's

National Nuclear Security Administration under Contract DE-AC04-94AL85000.

Approved for public release; further dissemination unlimited. 
Issued by Sandia National Laboratories, operated for the United States Department of Energy by Sandia Corporation.

NOTICE: This report was prepared as an account of work sponsored by an agency of the United States Government. Neither the United States Government, nor any agency thereof, nor any of their employees, nor any of their contractors, subcontractors, or their employees, make any warranty, express or implied, or assume any legal liability or responsibility for the accuracy, completeness, or usefulness of any information, apparatus, product, or process disclosed, or represent that its use would not infringe privately owned rights. Reference herein to any specific commercial product, process, or service by trade name, trademark, manufacturer, or otherwise, does not necessarily constitute or imply its endorsement, recommendation, or favoring by the United States Government, any agency thereof, or any of their contractors or subcontractors. The views and opinions expressed herein do not necessarily state or reflect those of the United States Government, any agency thereof, or any of their contractors.

Printed in the United States of America. This report has been reproduced directly from the best available copy.

Available to DOE and DOE contractors from

U.S. Department of Energy

Office of Scientific and Technical Information

P.O. Box 62

Oak Ridge, TN 37831

Telephone: $\quad$ (865) 576-8401

Facsimile: $\quad$ (865) $576-5728$

E-Mail: $\quad$ reports@adonis.osti.gov

Online ordering: http://www.osti.gov/bridge

Available to the public from

U.S. Department of Commerce

National Technical Information Service

5285 Port Royal Rd.

Springfield, VA 22161

Telephone:

(800) 553-6847

Facsimile:

(703) 605-6900

E-Mail:

orders@ntis.fedworld.gov

Online order:

http://www.ntis.gov/help/ordermethods.asp?loc=7-4-0\#online

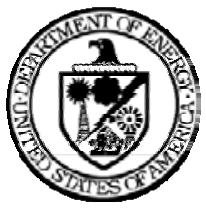


SAND2006-6763

Unlimited Release

Printed November 2006

\title{
Experimental Characterization of Spin Motor Nozzle Flow
}

\author{
Carl W. Peterson \\ John F. Henfling \\ Rocky J. Erven \\ Engineering Sciences Center \\ Sandia National Laboratories \\ P. O. Box 5800 \\ Albuquerque, NM 87185-0825
}

\begin{abstract}
The Mach number in the inviscid core of the flow exiting scarfed supersonic nozzles was measured using pitot probes. Nozzle characterization experiments were conducted in a modified section of an obsolete $\mathrm{M}=7.3$ test section/nozzle assembly on Sandia's Hypersonic Wind Tunnel. By capitalizing on existing hardware, the cost and time required for tunnel modifications were significantly reduced. Repeatability of pitot pressure measurements was excellent, and instrumentation errors were reduced by optimizing the pressure range of the transducers used for each test run. Bias errors in probe position prevented us from performing a successful in situ calibration of probe angle effects using pitot probes placed at an angle to the nozzle centerline.

The abrupt throat geometry used in the Baseline and Configuration A and B nozzles modeled the throat geometry of the flight vehicle's spin motor nozzles. Survey data indicates that small ("unmeasurable") differences in the nozzle throat geometries produced measurable flow asymmetries and differences in the flow fields generated by supposedly identical nozzles. Therefore, data from the Baseline and Configuration A and B nozzles cannot be used for computational fluid dynamics (CFD) code validation.

Configuration $\mathrm{C}$ and $\mathrm{D}$ nozzles replaced the abrupt throat geometry of Baseline and Configuration $\mathrm{A}$ and $\mathrm{B}$ nozzles with a 0.500 -inch streamwise radius of curvature in the throat region. This throat geometry eliminated the flow asymmetries, flow separation in the nozzle throat, and measurable differences between the flow fields from identical nozzles that were observed in Baseline/A/B nozzles. Data from Configuration $\mathrm{C}$ and $\mathrm{D}$ nozzles can be used for CFD code validation.
\end{abstract}




\section{Acknowledgments}

The authors wish to thank Paul Hooper and Douglas Hodge for their inputs in the design of the new facility hardware and instrumentation. We are also appreciative of the guidance regarding measurement uncertainty quantification provided by Bill Oberkampf. 


\section{Contents}

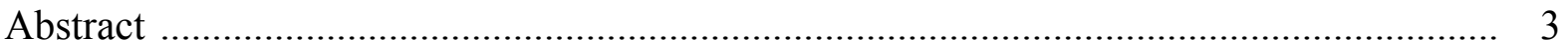

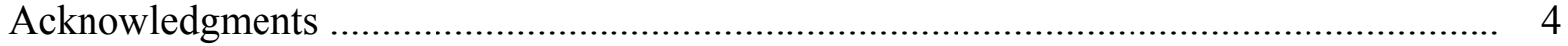

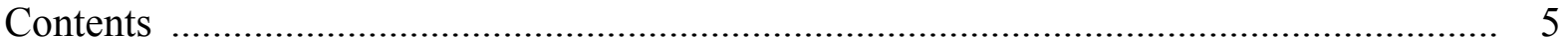

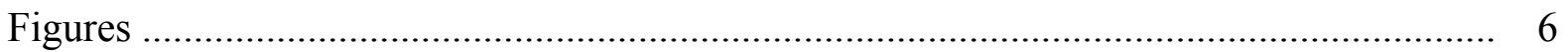

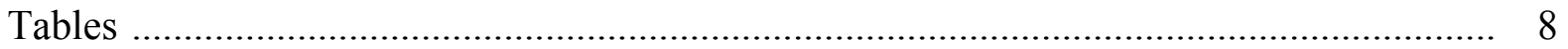

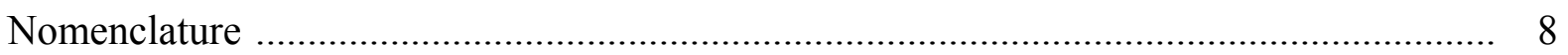

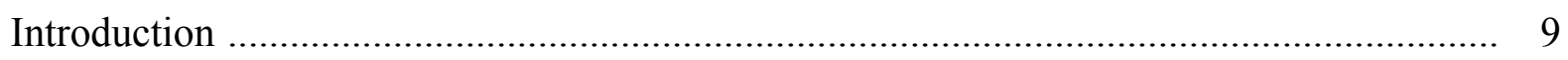

Spin Motor Nozzle Configurations ...................................................................... 11

Nozzle Characterization Experimental Setup ............................................................... 15

Sandia Hypersonic Wind Tunnel Modifications ............................................................ 15

Nozzle Operational Stagnation Temperature …………………………………..... 18

Pitot Probe Measurements ...................................................................................... 24

Measurement Error Sources and Accuracy ………………............................................ 26

Pressure Instrumentation Errors ……………………......................................... 26

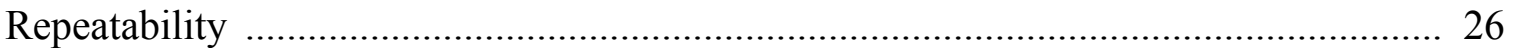

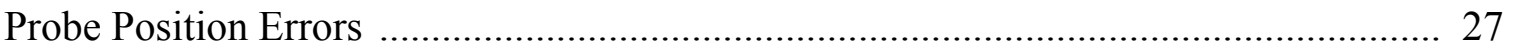

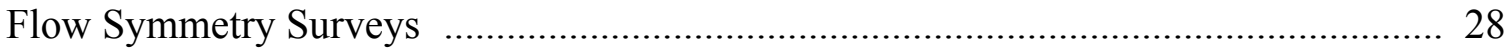

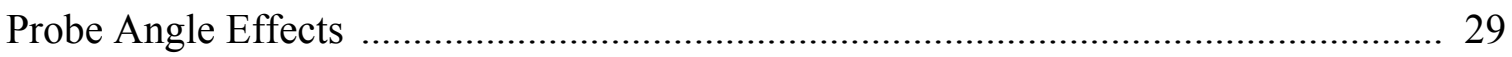

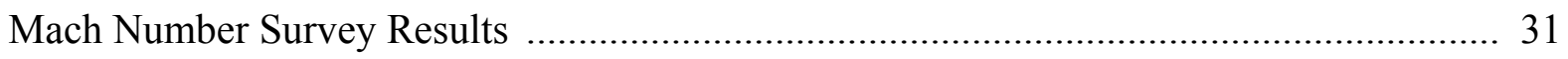

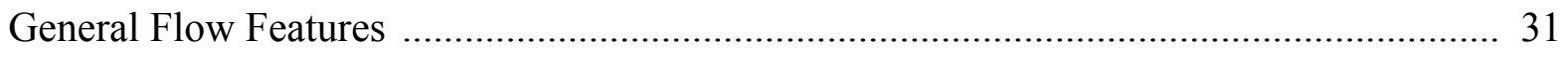

Centerline Mach Number Correlations with Stagnation Pressure …………………..... 31

Baseline Nozzle Survey Results ........................................................................... 34

Nozzle Configuration A and B Survey Results ……………………………………. 37

Nozzle Configuration C and D Survey Results ....................................................... 39

Summary and Conclusions ........................................................................................... 47

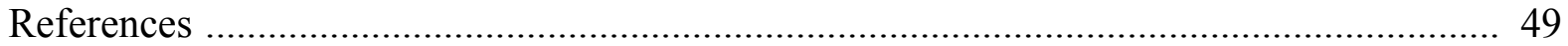

Appendix: Run Log for the Nozzle Characterization Experiments in the

Hypersonic Wind Tunnel ................................................................................... 51 


\section{Figures}

Figure 1: Spin motor plumes observed during a flight test ......................................... 9

Figure 2: Expected and observed spin rates from flight test ....................................... 9

Figure 3: Sketches of plume-freestream and vortex-fin interactions ............................. 10

Figure 4: Cross section sketch of the spin motor assembly in the flight vehicle ............ 11

Figure 5a: Full-scale wind tunnel model ................................................................ 11

Figure 5b: Balance adapter section details ............................................................. 12

Figure 5c: Cross-sectional view of the model nozzle in the AEDC model ..................... 12

Figure 6: Baseline spin motor model nozzle configuration ......................................... 12

Figure 7: Configuration A wind tunnel nozzle …………........................................ 12

Figure 8: Configuration $\mathrm{B}$ wind tunnel nozzle ........................................................ 12

Figure 9: Sketch of the throat region of Baseline and Configuration A and B Nozzles .. 13

Figure 10: Nozzle Configuration C ..................................................................... 14

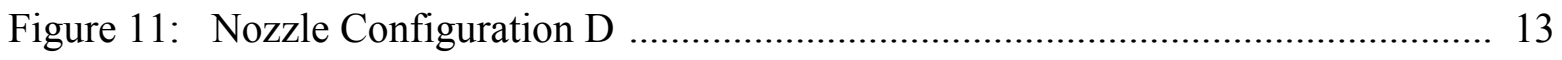

Figure 12: Sandia's Hypersonic Wind Tunnel facility ............................................... 15

Figure 13: Modifications made to the $\mathrm{M}=7.3 \mathrm{HWT}$ nozzle assembly .......................... 16

Figure 14: Nozzle test assembly and pitot probe drives mounted in the $M=7.3$ test section .................................................................................... 16

Figure 15: Photographs of the new nozzle characterization hardware upstream of the test section ................................................................................... 17

Figure 16: Nozzle flow characterization hardware during setup and in the $M=7.3$ test section …….................................................................................. 18

Figure 17: Variation of minimum stagnation temperature required to avoid nitrogen gas saturation in the model spin motor nozzles ................................................. 20

Figure 18: Variation of minimum stagnation temperature required to avoid nitrogen gas supersaturation in the model spin motor nozzles .......................................... 20

Figure 19: Centerline Mach number surveys for Nozzle C-40 for $Z=0.010$ inches and $64 \leq P_{0} \leq 950 \mathrm{lbs} / \mathrm{in}^{2}$

Figure 20a: Centerline Mach number surveys for Nozzle D-30 for $Z=0.010$ inches and $62 \leq P_{0} \leq 980 \mathrm{lbs} / \mathrm{in}^{2}$

Figure 20b: Centerline Mach number surveys for Nozzle D-30 for $Z=0.460$ inches and $62 \leq P_{0} \leq 481 \mathrm{lbs} / \mathrm{in}^{2}$

Figure 21: Repeated surveys on the $\mathrm{Y}=0$ plane at six axial locations downstream of Nozzle C-40, $P_{0}=250 \mathrm{lbs} / \mathrm{in}^{2}$

Figure 22: Comparison of Nozzle D-31 Mach number surveys along the $X=0$ and $\mathrm{Y}=0$ axes for (bottom to top) $\mathrm{Z}=0.010,0.160,0.310$ and 0.460 in., $P_{0}=500 \mathrm{lbs} / \mathrm{in}^{2}$

Figure 23: Comparisons of centerline Mach number surveys for probe cant angles of 0 and 5 degrees; Nozzle D-30, $P_{0}=250 \mathrm{lbs} / \mathrm{in}^{2}$ 
Figure 24: Schlieren photograph of the Baseline nozzle flow field

Figure 25: Comparison of centerline axial Mach number $(\mathrm{X}=\mathrm{Y}=0)$ for Baseline Nozzle 0 (before polishing) and Baseline Nozzle 0-2 (after polishing)

Figure 26: Comparison of Baseline Nozzle 0-2 and Nozzle 1 Mach number surveys on the $\mathrm{Y}=0$ plane for $\mathrm{Z}=0.015$ inches and $P_{0}=100 \mathrm{lbs} / \mathrm{in}^{2}$

Figure 27: Mach number surveys in the $Y=0$ plane at $Z=0.010$ inches for Baseline Nozzle 0-2

Figure 28: Mach number surveys in the $\mathrm{Y}=0$ plane at $\mathrm{Z}=0.010$ inches for Baseline Nozzle 1

Figure 29: Nozzle exit plane Mach number surveys for Baseline Nozzle 0-2 ............... 36

Figure 30: Nozzle exit plane Mach number surveys for Baseline Nozzle 1 .................. 36

Figure 31: Mach number surveys in the $\mathrm{Y}=0$ plane at $\mathrm{Z}=0.010$ inches for Nozzle Configuration A-10

Figure 32: Mach number surveys in the $\mathrm{Y}=0$ plane at $\mathrm{Z}=0.010$ inches for Nozzle Configuration A-11-2

Figure 33: Mach number surveys in the $\mathrm{Y}=0$ plane at $\mathrm{Z}=0.010$ inches for Nozzle Configuration B-20-2

Figure 34: Mach number surveys in the $\mathrm{Y}=0$ plane at $\mathrm{Z}=0.010$ inches for Nozzle Configuration B-21-2

Figure 35: Nozzle Configuration C-40 centerline exit Mach number survey results ....... 40

Figure 36: Nozzle Configuration C-41 centerline exit Mach number survey results ....... 40

Figure 37: Nozzle Configuration D-30 centerline exit Mach number survey results ....... 41

Figure 38: Nozzle Configuration D-31 centerline exit Mach number survey results ....... 41

Figure 39a Comparison of Baseline and Configuration $\mathrm{C}$ nozzle centerline exit Mach number surveys, $P_{0} \cong 62 \mathrm{lbs} / \mathrm{in}^{2}$

Figure 39b Comparison of Baseline and Configuration C nozzle centerline exit Mach number surveys, $P_{0} \cong 250 \mathrm{lbs} / \mathrm{in}^{2}$

Figure 39c Comparison of Baseline and Configuration C nozzle centerline exit Mach number surveys, $P_{0} \cong 935 \mathrm{lbs} / \mathrm{in}^{2}$

Figure 40a Comparison of Configuration B and D nozzle centerline exit Mach number surveys, $P_{0} \cong 62 \mathrm{lbs} / \mathrm{in}^{2}$

Figure 40b Comparison of Configuration $\mathrm{B}$ and $\mathrm{D}$ nozzle centerline exit Mach number surveys, $P_{0} \cong 250 \mathrm{lbs} / \mathrm{in}^{2}$

Figure 40c Comparison of Configuration B and D nozzle centerline exit Mach number surveys, $P_{0} \cong 1000 \mathrm{lbs} / \mathrm{in}^{2}$

Figure 41: Location of pitot probes for surveys across the nozzle exit plane

Figure 42: Mach number profiles obtained at twelve $\mathrm{Y}$ locations across the Configuration C-40 nozzle exit plane, $P_{0}=150 \mathrm{lbs} / \mathrm{in}^{2}$... 46

Figure 43: Mach number profiles obtained at twelve $Y$ locations across the Configuration D-30 nozzle exit plane, $P_{0}=500 \mathrm{lbs} / \mathrm{in}^{2}$ 


\section{Tables}

Table 1: Minimum stagnation temperatures for avoiding nitrogen condensation at the nozzle exit for $P_{0}=1000 \mathrm{lbs} / \mathrm{in}^{2}$

Table 2: Average standard deviation in Mach number for repeated surveys and symmetry comparisons

Table 3: Polynomial coefficients for correlating centerline nozzle exit Mach number with $P_{0}$ for Configuration $\mathrm{C}$ and $\mathrm{D}$ nozzles at $\mathrm{Z}=0.010$ inches

Table 4: Polynomial coefficients for correlating centerline nozzle exit Mach number with $P_{0}$ for the Baseline and Configuration $\mathrm{A}$ and $\mathrm{B}$ nozzles at $\mathrm{Z}=0.0$ inches

\section{Nomenclature}

$J$ plume-freestream interaction parameter, $Q_{\text {nozzle }} / Q_{\infty}$

$M$ Mach number

$M_{n o z}$ Centerline Mach number at the nozzle exit determined from experimental data and correlated to nozzle stagnation pressure (Eq. 4)

$P_{0}$ nozzle stagnation pressure, $1 \mathrm{bs} / \mathrm{in}^{2}$ (psia)

$P_{t}$ Pitot pressure, $\mathrm{lbs} / \mathrm{in}^{2}$ (psia)

$Q_{\text {nozzle }}$ dynamic pressure on the nozzle centerline at the nozzle exit

$Q_{\infty}$ freestream dynamic pressure of the flight vehicle, either in flight or in the wind tunnel

$T_{0}$ nozzle stagnation temperature, degrees Rankine

$X$ dimension across scarfed nozzle face, inches

$Y$ vertical nozzle dimension, inches

$Z$ axial nozzle dimension, inches

$Z^{\prime}$ distance downstream of the nozzle exit plane, inches

$\gamma$ ratio of specific heats 


\section{Experimental Characterization of Spin Motor Nozzle Flow}

\section{Introduction}

Spin motors are used on gravity bombs and other finned vehicles to achieve an elevated spin rate immediately after release of the vehicle from the delivery aircraft (Fig. 1). A fluid dynamic interaction occurs when the airflow past the vehicle sweeps the spin motor plumes downstream and forms vortices during the interaction with the plume. This plumefreestream flow interaction is called "jet-in-crossflow" in the literature. The vortices created by the jet-in-crossflow interaction can induce pressures on the payload's fins (called "vortexfin interaction" in this report). The induced pressure field can generate a "counter torque" in opposition to the torque produced by the spin motor and fin cant. The counter torque results in a reduction of the spin rate of the vehicle (Fig. 2). Figure 3 is a sketch of the vortex-fin interaction process, including the plume and vortices, the induced fin pressures generated during motor firing, and the resulting "counter torque" in the opposite direction of the spin motor torque.

Research on plume/freestream interaction for axisymmetric nozzles ${ }^{1-8}$ indicates that the nondimensional parameter $J$ has been successful in correlating plume-freestream interaction phenomena such as plume penetration into the freestream. $J$ is defined by

$$
J \equiv Q_{\text {nozzle }} / Q_{\infty},
$$

where $Q_{\text {nozzle }}$ is the dynamic pressure on the nozzle centerline where it intersects the vehicle surface and $Q_{\infty}$ is the dynamic pressure of the airflow past the flight vehicle. However, neither experimental data nor validated Computational Fluid Dynamics (CFD) predictions could be found that characterized the primary fluid dynamic and vehicle configuration parameters affecting the magnitude of the loss of spin rate caused by vortex-fin interaction. To fill this void, full-scale wind tunnel tests in Arnold Engineering Development Center's 16-ft Transonic

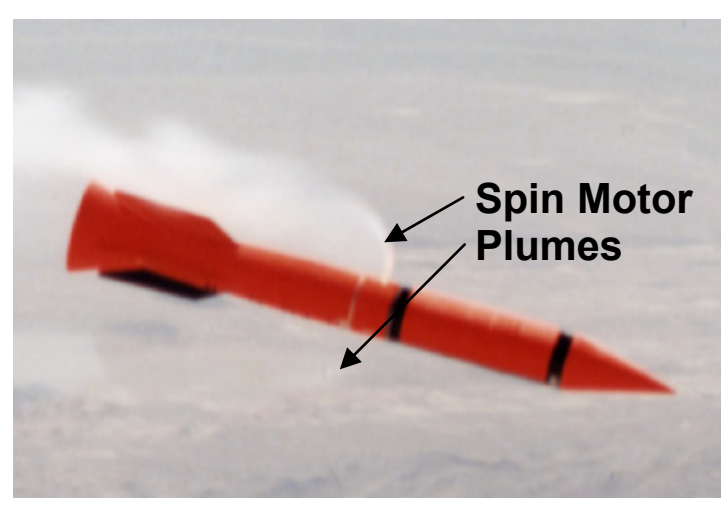

Figure 1. Spin motor plumes observed during a flight test.

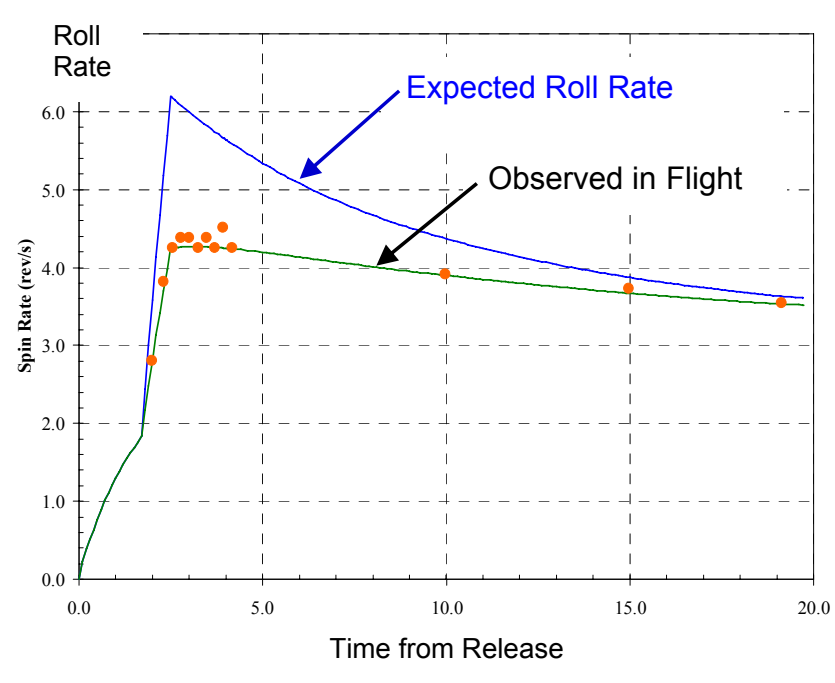

Figure 2. Expected and observed spin rates from flight test. 


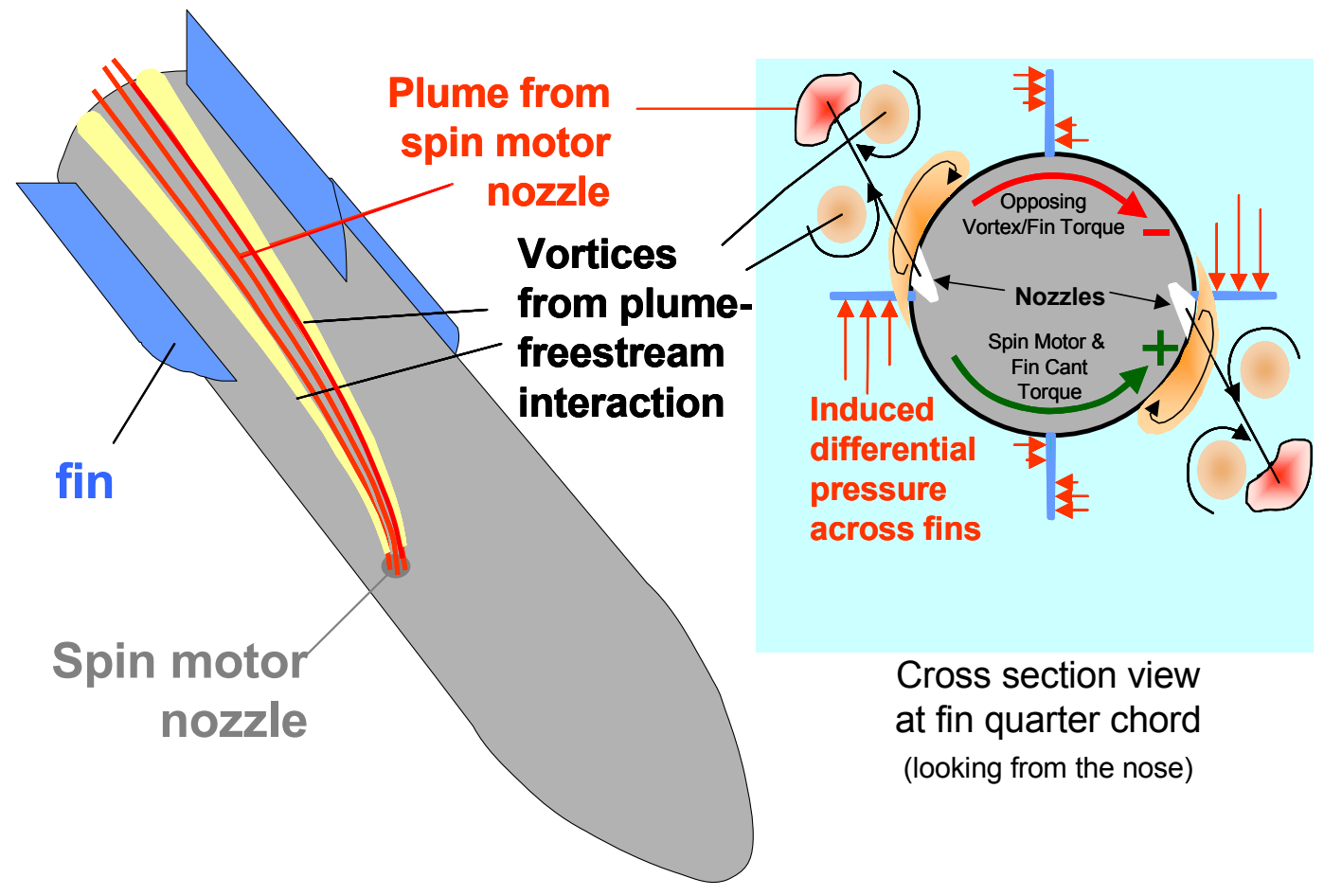

Figure 3. Sketches of plume-freestream and vortex-fin interactions.

Wind Tunnel and in NASA Ames Research Center's 11-ft Unitary Transonic Wind Tunnel were conducted to measure the counter torque caused by vortex-fin interaction for a variety of flight conditions (dynamic pressure, Mach number and Reynolds number), $J$ values and vehicle configuration parameters.

In order to correlate the wind tunnel vortex-fin interaction data on $J$, the value of $Q_{\text {nozzle }}$ had to be known for each supersonic spin motor nozzle configuration used in the full-scale vortex-fin interaction wind tunnel experiments. This report describes the facilities and experimental techniques used to determine $Q_{\text {nozzle }}$ by characterizing the flow at the nozzle exit plane. In addition to the nozzle flow characterization measurements, detailed flow field surveys were made on a few nozzles in order to provide a validation data set for comparison to predictions of nozzle exit flow properties from Sandia compressible CFD codes. Experiments for identifying, isolating and quantifying experimental error sources were conducted. In-situ calibrations of probe angle-of-attack effects on pitot pressure measurements were performed in order to estimate the flow angle at the exit of the nozzles. 


\section{Spin Motor Nozzle Configurations}

Figure 4 shows the position of the two spin motor nozzles on opposite sides of the flight vehicle. They are canted at an angle of $39.5^{\circ}$ with respect to the vehicle surface normal. The nozzle exit plane is defined by the intersection of the conical nozzle wall with the surface of the 13.3-inch-diameter flight vehicle. The nozzles used in the actual rocket motor have a nozzle exit diameter of 1.504 inches (measured on the $Y=0$ plane; see Fig. 6 for definition of the nozzle coordinate system), a throat diameter of 0.390 inches and a nozzle wall expansion half-angle of $15^{\circ}$. No one has ever measured the gas composition or the flow field properties coming out of the flight vehicle spin motors. The manufacturers of the spin motors (ATK) believe that the combusted gas is comprised primarily of water vapor. ATK estimates that the gas temperature is $\sim 2575 \mathrm{~K}$ $\left(4635^{\circ} \mathrm{R}\right.$ ) and the pressure is $\sim 1200 \mathrm{lbs} / \mathrm{in}^{2}$ in the motor's combustion chamber. It is not known whether the gas is in equilibrium or is frozen as it expands through the nozzle, so we assumed a value of the ratio of specific heats $(\gamma)$ of 1.23 , which is the average of equilibrium and frozen values. Based upon the ratio of exit area to throat area, the calculated Mach number on the nozzle centerline at the exit plane of the flight spin motor nozzles is 3.66. The nozzle wall boundary layer displacement thickness will make the actual (observed) Mach number lower than

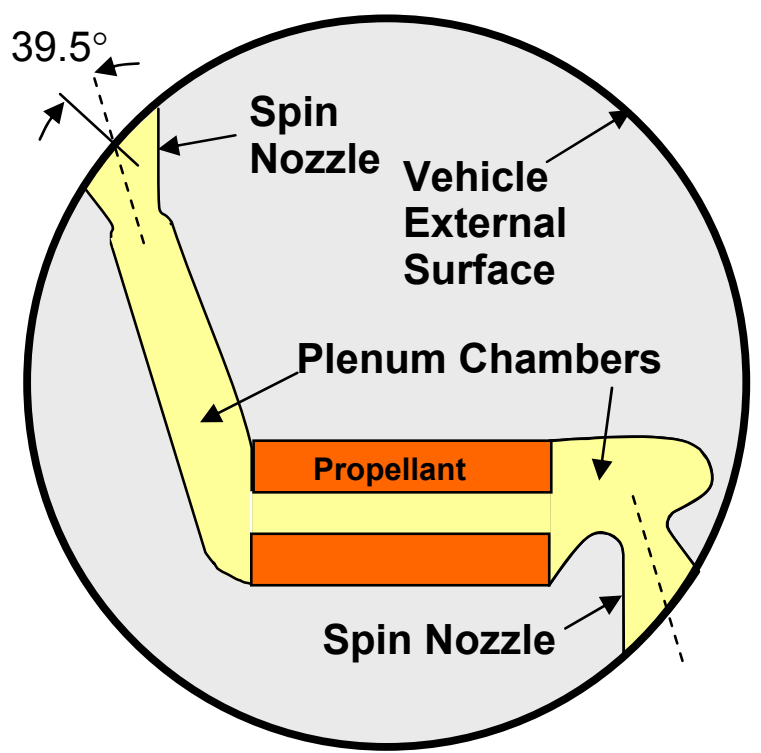

Figure 4. Cross section sketch of the spin motor assembly in the flight this value.

Actual flight vehicle spin motors were not used in the full-scale wind tunnel experiments because only one data point could be obtained for each motor firing and because of the long down time that would have been needed to replace expended motors between runs. Therefore, the full-scale wind tunnel models were designed to use high-pressure air instead of the combustion products produced by the flight vehicle's spin motors. Figure 5 presents three views of the wind tunnel model, the balance adapter section, and the special nozzles used in the wind tunnel model. Air is fed to the nozzles from an air supply located outside of the specifically for these experiments by AEDC. After passing through the balance, the air passageway bifurcates and directs equal quantities of air to the stagnation chamber upstream of each nozzle.

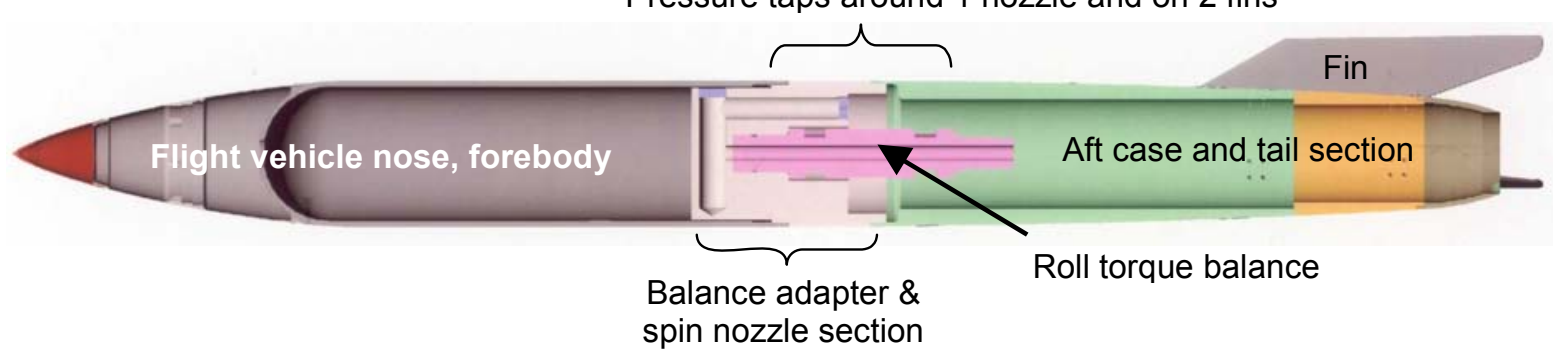

Figure 5a. Full-scale wind tunnel model. 


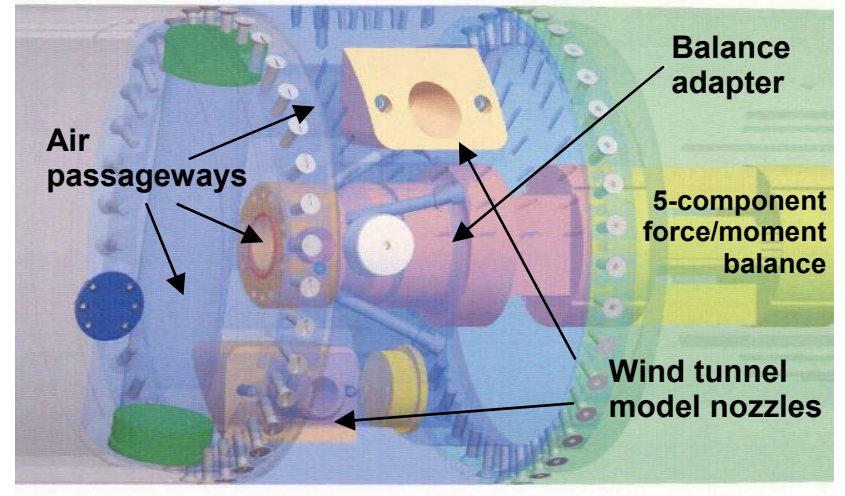

Figure 5b. Balance adapter section details.

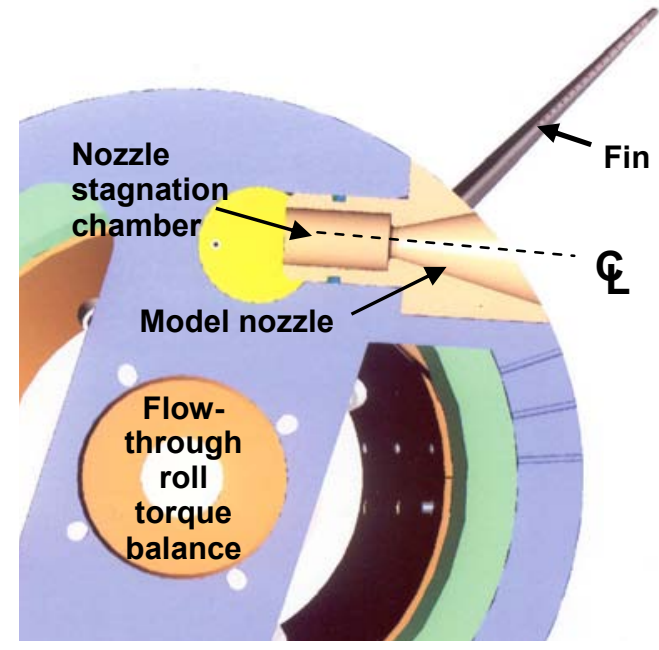

Figure 5c. Cross-sectional view of the spin motor in the wind tunnel model.
The flight vehicle spin nozzles were replaced by baseline wind tunnel model nozzles that had the same throat geometry and exit dimensions as the nozzles used on the flight vehicle, but the throat diameter was increased from 0.390 inches to 0.535 inches in order to generate the same exit centerline Mach number using air (for which $\gamma=1.4$ ) as was calculated for the flight vehicle nozzles (assuming $\gamma$ $=1.23$ ). Figure 6 shows the baseline nozzle contour and the nozzle coordinate system used in the nozzle flow surveys. The $X$ axis is perpendicular to the page. The nozzle centerline is the axis of the conical nozzle, and the nozzle exit centerline location is defined as $X=Y=Z=0$.

In addition to the baseline nozzle, two other nozzle configurations were used in the AEDC experiments. Configuration A (Fig. 7) was a scaled-down baseline nozzle with a throat diameter of 0.476 inches (instead of 0.535 inches) and an exit diameter on the $Y=0$ plane of 1.338 inches. Configuration B (Fig. 8) also had a throat diameter of 0.476 inches, but it retained the same exit dimensions as those of the baseline nozzle. The expansion half angle was kept at $15^{\circ}$. The baseline nozzle and the two additional nozzle configurations were used in

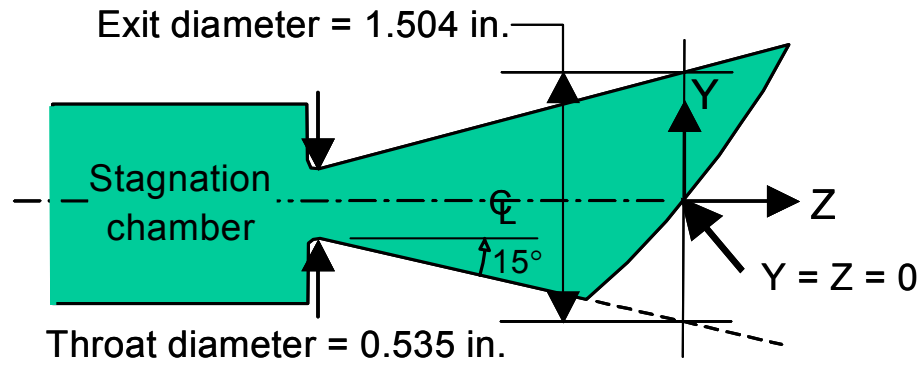

Figure 6. Baseline spin motor model nozzle configuration.

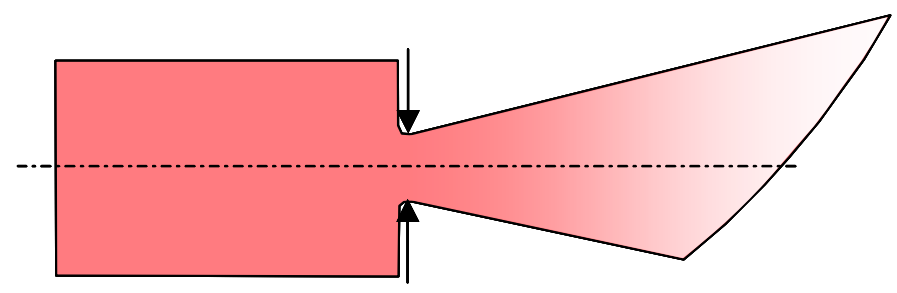

Throat diameter $=0.476 \mathrm{in}$.

Figure 7. Configuration A wind tunnel nozzle; scaled down version of the baseline nozzle.

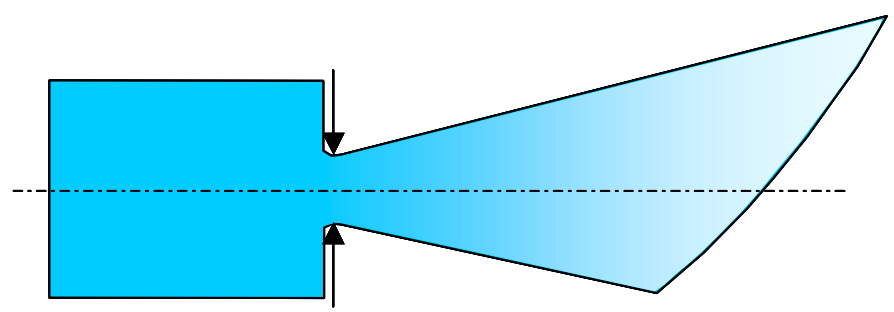

Throat diameter $=0.476$ in.

Figure 8. Configuration B wind tunnel nozzle; same exit dimensions as baseline nozzle. 
the AEDC wind tunnel experiments. Of these three nozzles, only the baseline nozzle was used in the NASA Ames wind tunnel experiments.

In each of these nozzle configurations, the throat region geometry approximated that of the flight nozzles, which were designed to minimize manufacturing cost and therefore did not incorporate design features that promoted flow quality. Figure 9 is a sketch of the throat region of the baseline and Configuration $\mathrm{A}$ and $\mathrm{B}$ nozzles. Upstream of the throat, the wall of the model nozzles was oriented at $90^{\circ}$ to the nozzle axis. The radius of curvature leading to the throat was less than 0.020 inches, and the throat itself was $\sim 0.015$ inches long. The abrupt changes in flow direction caused by such small radius of curvature and $90^{\circ}$ angular changes of direction promotes separation of the flow in the throat region and caused the resulting nozzle exit flow to depend upon throat physical dimensions that cannot be measured (i.e., very small geometric perturbations lead to measurable differences in the flow). Nozzles with identical dimensional inspection reports produced slightly different flow, as will be shown later in this report.

Therefore, a fourth nozzle configuration (Configuration C, Fig. 10) was constructed with similar throat, nozzle expansion angle and exit dimensions as the baseline nozzle, but with a 0.500 -inch-diameter streamwise radius of curvature through the throat region to avoid flow separation in the throat region. Nozzle Configuration D (Fig. 11) retained the same exit dimensions as those of configuration $\mathrm{C}$ but had a throat diameter of 0.480 inches. Because of their larger nozzle expansion area ratio, nozzles B and D produced higher exit Mach numbers than the other nozzles. Configuration $\mathrm{C}$ and $\mathrm{D}$ nozzles were constructed after the AEDC wind tunnel entry, so they were only used in the NASA Ames wind tunnel experiments.

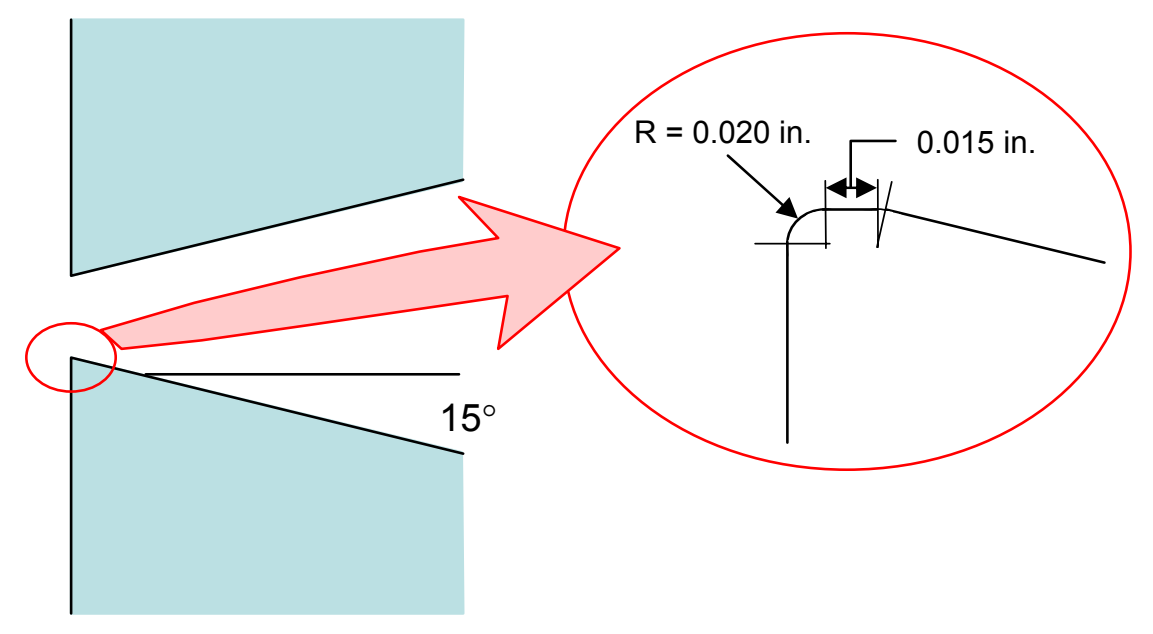

Figure 9. Sketch of the throat region of Baseline and Configuration A and B nozzles. 


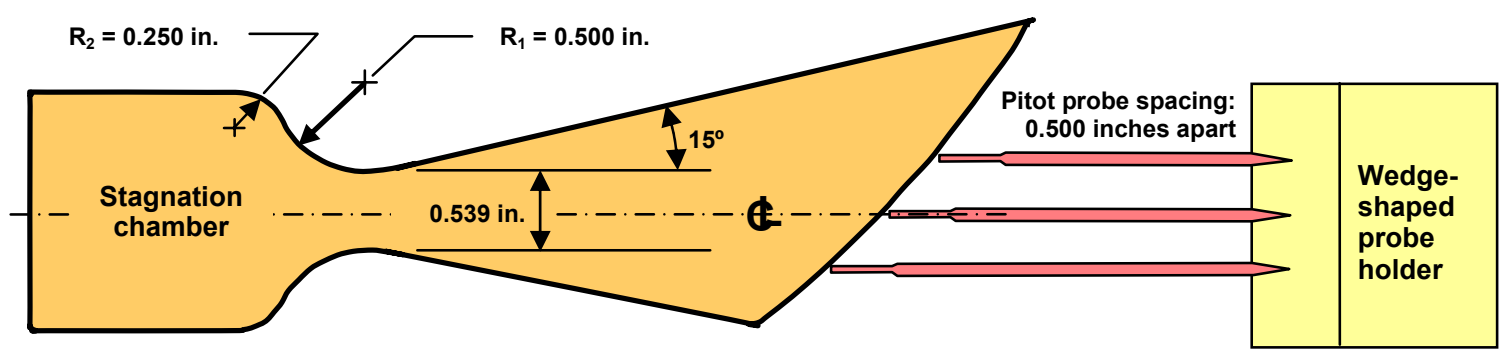

Figure 10. Nozzle Configuration C; similar dimensions as baseline nozzle with a 0.500 -inch streamwise radius of curvature in the throat region.

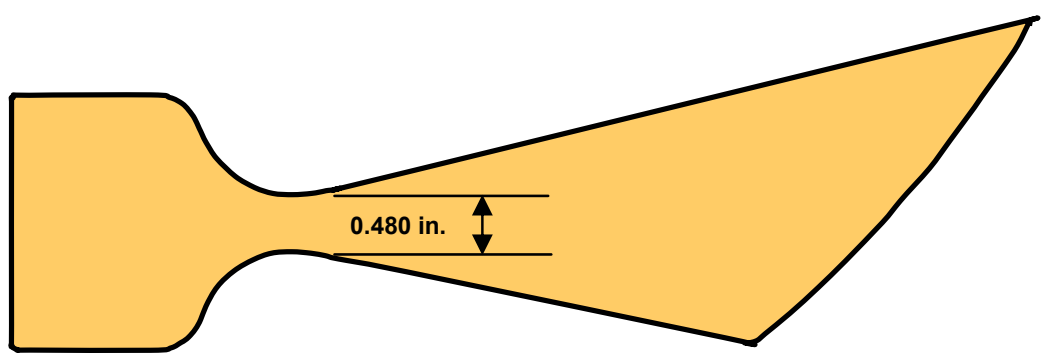

Figure 11. Nozzle Configuration D; similar dimensions as Configuration B nozzle with a 0.500 -inch streamwise radius of curvature in the throat region. 


\section{Nozzle Characterization Experimental Setup}

\section{Sandia Hypersonic Wind Tunnel Modifications}

Because we wanted to make pitot pressure measurements in the exit plane of the wind tunnel nozzles before the May 2002 AEDC wind tunnel entry, we needed access to an existing Sandia facility that could be modified quickly. Additional requirements of the facility included the following:

- Make available air or nitrogen gas at pressures from $62.5 \mathrm{lbs} / \mathrm{in}^{2}$ to $1000 \mathrm{lbs} / \mathrm{in}^{2}$ at a mass flow rate of $5 \mathrm{lbm} / \mathrm{sec}$ when the pressure is $1000 \mathrm{lbs} / \mathrm{in}^{2}$.

- Exhaust into a chamber whose ambient pressure can be maintained at a level below the centerline static pressure at the nozzle exit.

- Allow pitot probe measurements to be made in three dimensions across the exit plane of the nozzle, following the cylindrical contour of the model surface.

- Contain the noise of high-pressure supersonic nozzle operation.

Sandia's Hypersonic Wind Tunnel (HWT, Figure 12) stores five separate hypersonic nozzles and test sections ("legs") on a rotary support system. The rotary system connects any of these test section/nozzle combinations to its gas supply (air or nitrogen, depending upon the Mach number) and the vacuum system (three spheres with a total volume of 30,000 $\mathrm{ft}^{3}$ ). The $M=5,8$ and 14 HWT legs are currently active. The $M=8$ leg uses nitrogen gas at pressures up to $1400 \mathrm{lbs} / \mathrm{in}^{2}$ and at mass flow rates of up to $9.75 \mathrm{lbm} / \mathrm{sec}$. The $\mathrm{M}=14$ leg uses nitrogen gas at pressures up to $2900 \mathrm{lbs} / \mathrm{in}^{2}$ and at mass flow rates of up to $\sim 1.8 \mathrm{lbm} / \mathrm{sec}$. In these experiments, the HWT's $30,000-\mathrm{ft}^{3}$ vacuum system allows nearly continuous operation at the lowest nozzle stagnation pressures, and the $8500 \mathrm{lbs} / \mathrm{in}^{2}, 62 \mathrm{ft}^{3}$ nitrogen storage system allows run times at the highest stagnation pressures $\left(800-1000 \mathrm{lbs} / \mathrm{in}^{2}\right)$ of $\sim 40$ seconds.

The HWT's $M=7.3$ and $M=11$ nozzles were installed when the HWT was first made operational in $1962^{9}$. However, these nozzles have not been used since the HWT's original pebble bed heater was decommissioned in 1975 . They were left on the rotary nozzle support structure as counterweights to balance the weight of the operational nozzles. We decided to modify the $\mathrm{M}=7.3 \mathrm{HWT}$ nozzle assembly in order to capitalize on existing wind tunnel hardware and infrastructure needed to make the nozzle exit flow measurements.

Figures 13 and 14 show the modifications made to the $\mathrm{M}=7.3$ nozzle assembly in order to locate the wind tunnel model spin motor nozzles in the test section and connect them to the existing nitrogen gas supply and vacuum system. The $\mathrm{M}=7.3$ stagnation chamber was

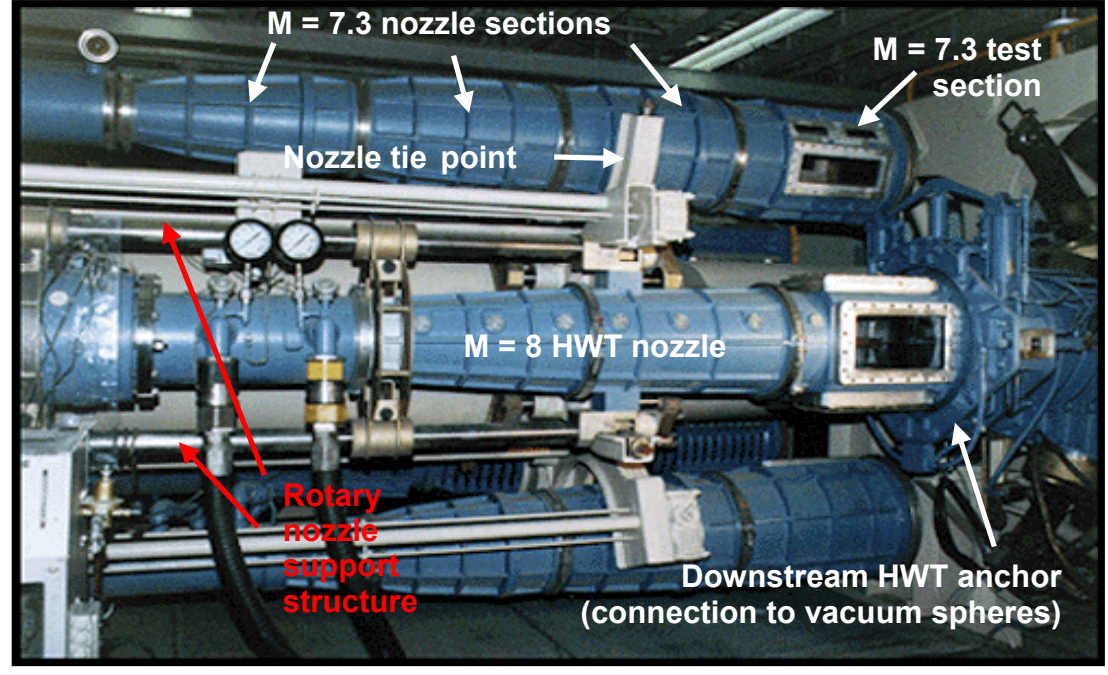

Figure 12. Sandia's Hypersonic Wind Tunnel facility. 


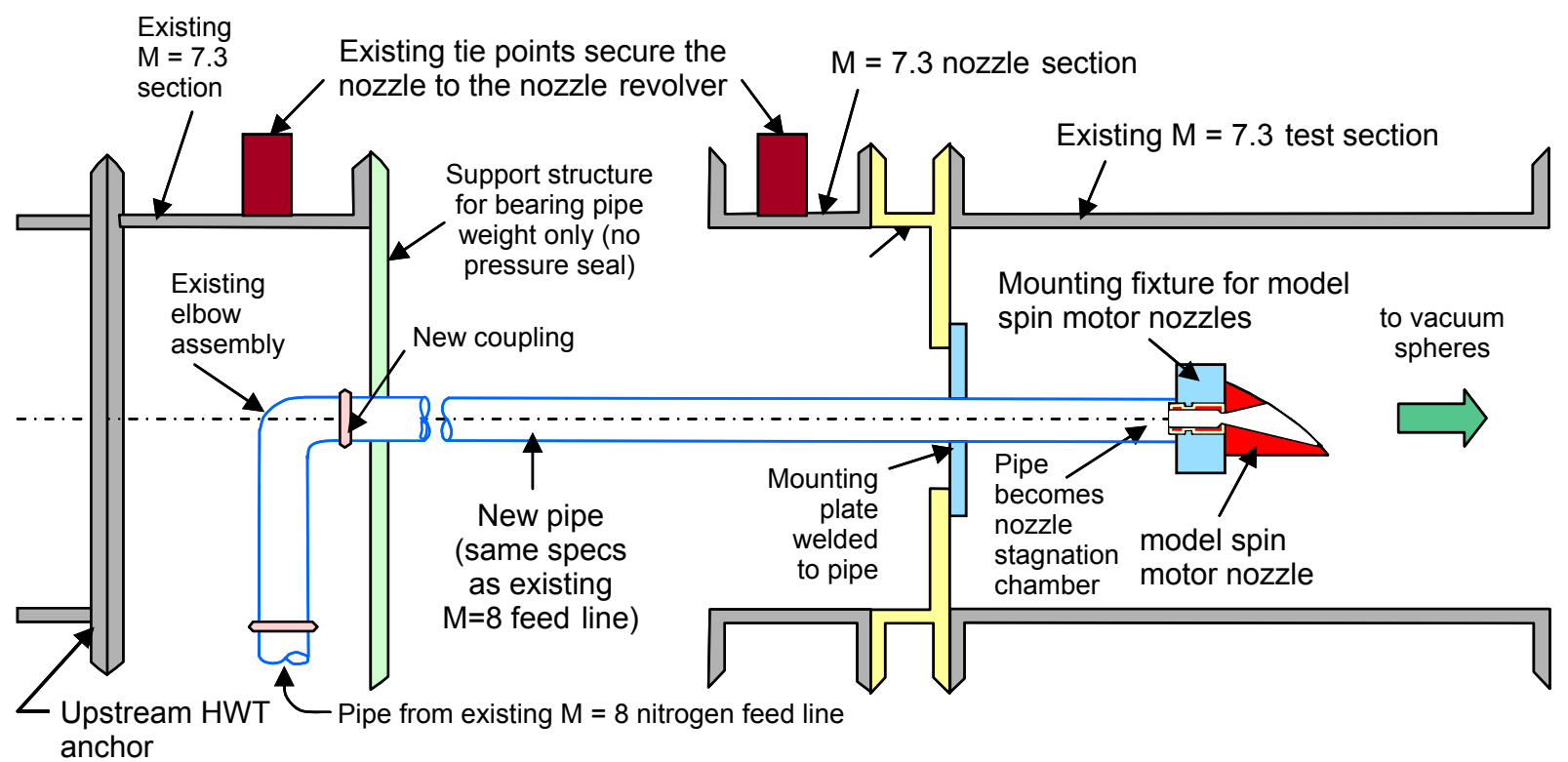

Figure 13. Modifications made to the $M=7.3$ HWT nozzle assembly.

retained because it provides a tie to the upstream HWT anchor point and is firmly secured to the rotary nozzle support structure (the "nozzle revolver"). A portion of the $M=7.3$ stagnation chamber wall was cut out to allow the existing feed line from the $\mathrm{M}=8$ nitrogen nozzle to reach the centerline of the nozzle assembly. The existing $\mathrm{M}=8$ elbow assembly aligns the nitrogen feed line with the tunnel centerline. Hardware that served no future purpose was removed. The stagnation chamber was also fitted with a support structure for bearing the weight of the elbow and the 2-inch-dia. Schedule 160304 stainless steel pipe that leads to the fixture for mounting the model nozzles. This is the same specification of pipe that is used to bring nitrogen into the $\mathrm{M}=8$ electric heater.

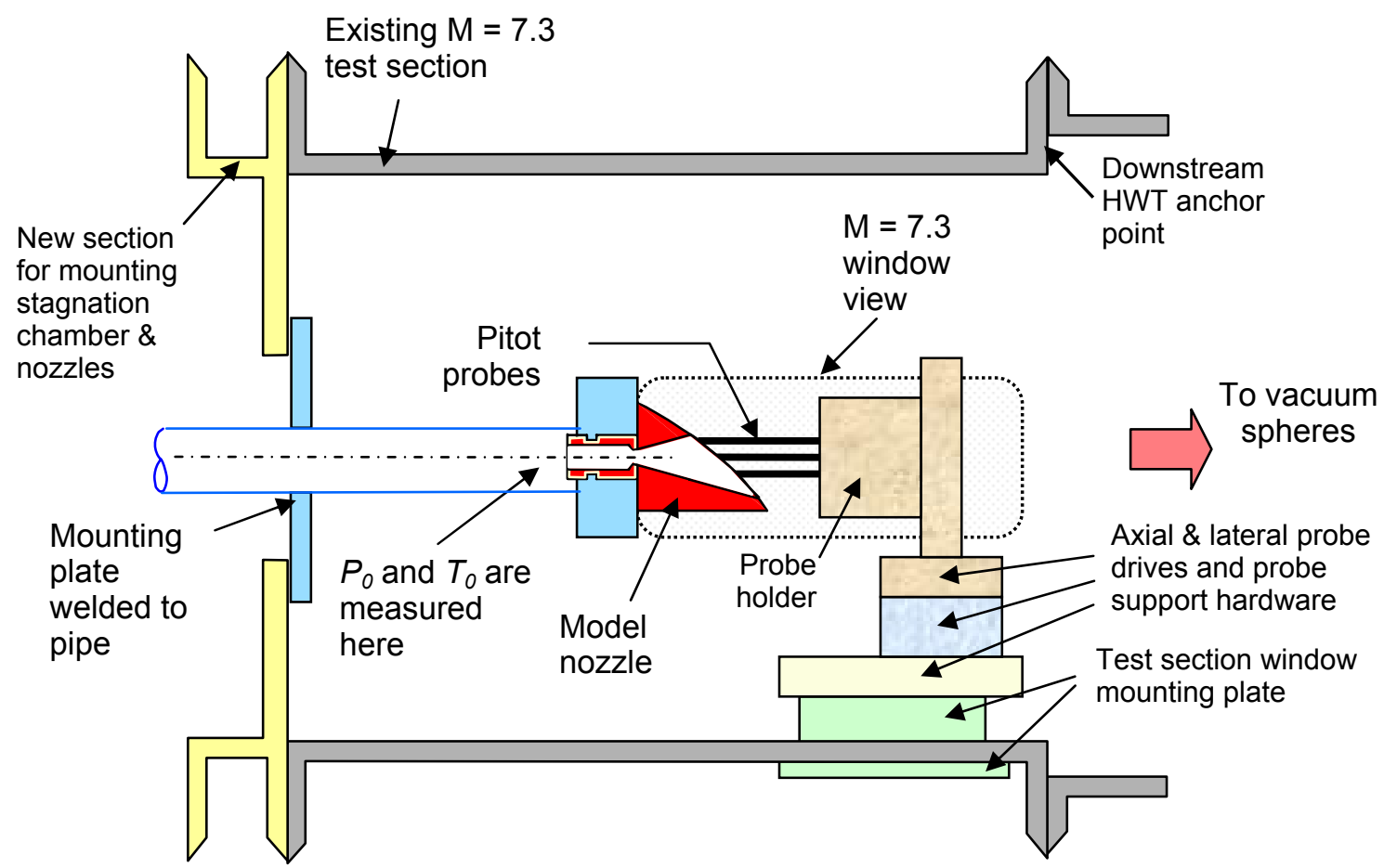

Figure 14. Nozzle test assembly and pitot probe drives mounted in the $M=7.3$ test section. 
The first three of the four $M=7.3$ nozzle expansion sections (including the throat section) were removed from the nozzle revolver to make room for hardware that allows the full-scale wind tunnel model nozzles to be mounted in the $M=7.3$ test section. Three angle iron sections (not shown in the sketches or photos) connect the fourth $\mathrm{M}=7.3$ nozzle section to the $\mathrm{M}=7.3$ stagnation chamber to transmit the $3000-\mathrm{lb}$ tension loads to the upstream anchor point when the test section is evacuated. The fourth $M=7.3$ nozzle section was retained because it is anchored to the rotary nozzle revolver. The $\mathrm{M}=7.3$ test section was also retained because it provides the connection to the downstream anchor point (Figure 14) and vacuum spheres, and because it has four window ports for mounting pitot probe drives and taking Schlieren photographs of the nozzle exit flow. Figure 14 presents photographs of the modified nitrogen line elbow and feed line upstream of the new wind tunnel section.

A new section was inserted between the last $\mathrm{M}=7.3$ nozzle expansion section and the $M=7.3$ test section (see Figures 13 and 14). Its purpose is to support the nozzle flow characterization hardware, which consists of the nitrogen gas feed pipe and the fixture to which the wind tunnel model nozzles are mounted. The pipe brings the nitrogen gas from the existing $M=8$ feed line and elbow to the nozzle attachment fixture in the $M=7.3$ test section. Stagnation temperature and pressure are measured in the pipe just upstream of the nozzle attachment fixture, which is positioned in the $M=7.3$ test section such that both the nozzles and the flow from the nozzles are in the field of view of the existing window ports (Figure 14). The nozzle is attached to the fixture with two bolts. Each nozzle can be rolled with respect to the pipe axis by \pm 90 and 180 degrees relative to the roll orientation shown in Figure 14 to obtain pitot surveys for evaluating the symmetry of the nozzle flow. Figures 15 and 16 show the nozzle flow characterization hardware mounted on the HWT nozzle revolver and new wind tunnel section.

The $\mathrm{M}=8$ pressure control system was used to set nozzle stagnation pressures above 500 lbs/in ${ }^{2}$ (at high mass flow rates), and the $\mathrm{M}=14$ system was used to control $P_{0}$ below 500 lbs/in ${ }^{2}$ (at low mass flow rates). Some changes were made to the control system in recognition that neither the $\mathrm{M}=8$ nor the $\mathrm{M}=14$ electric heaters are used in this experiment. Environmental, health and safety $(\mathrm{ES} \& H)$ issues were a benign subset of those needed to run the $\mathrm{M}=8 / 14$ facilities because the present experiment was conducted at pressures below their maximum allowable working pressures. Safety interlocks pertaining to the $M=8 / 14$ electric
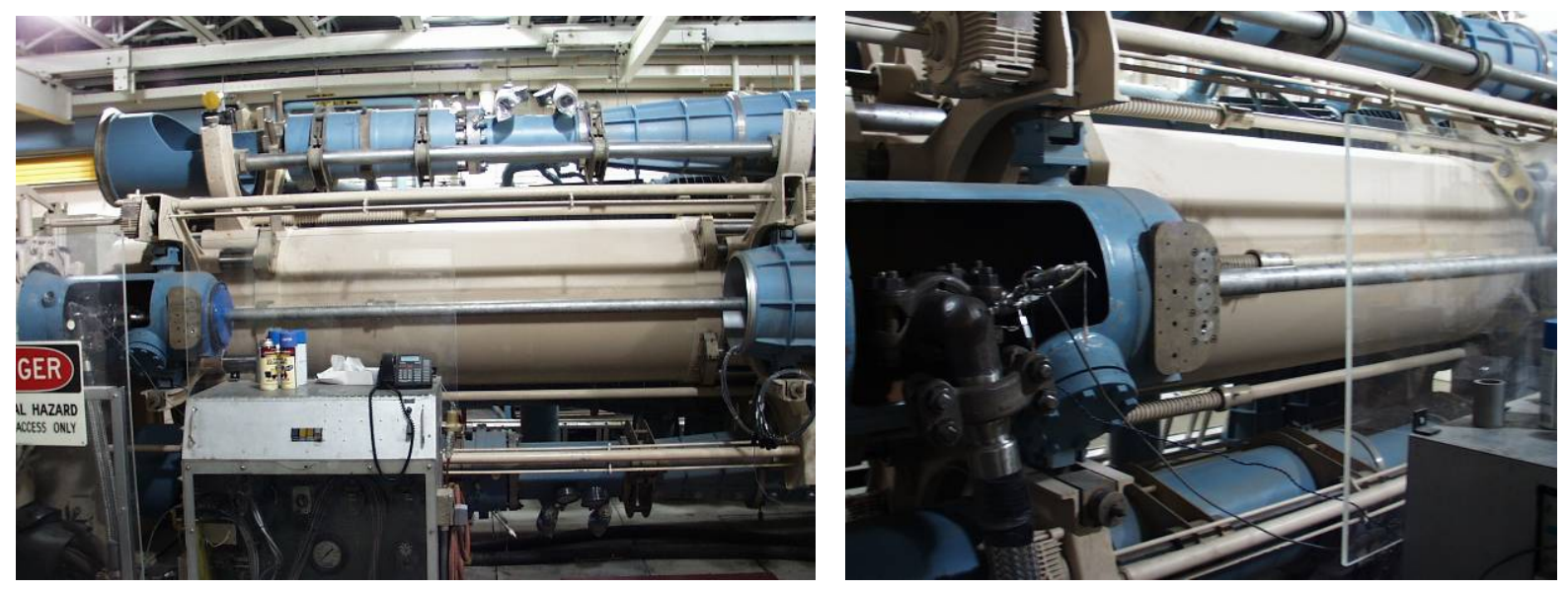

Figure 15. Photographs of the new nozzle characterization hardware upstream of the test section. 


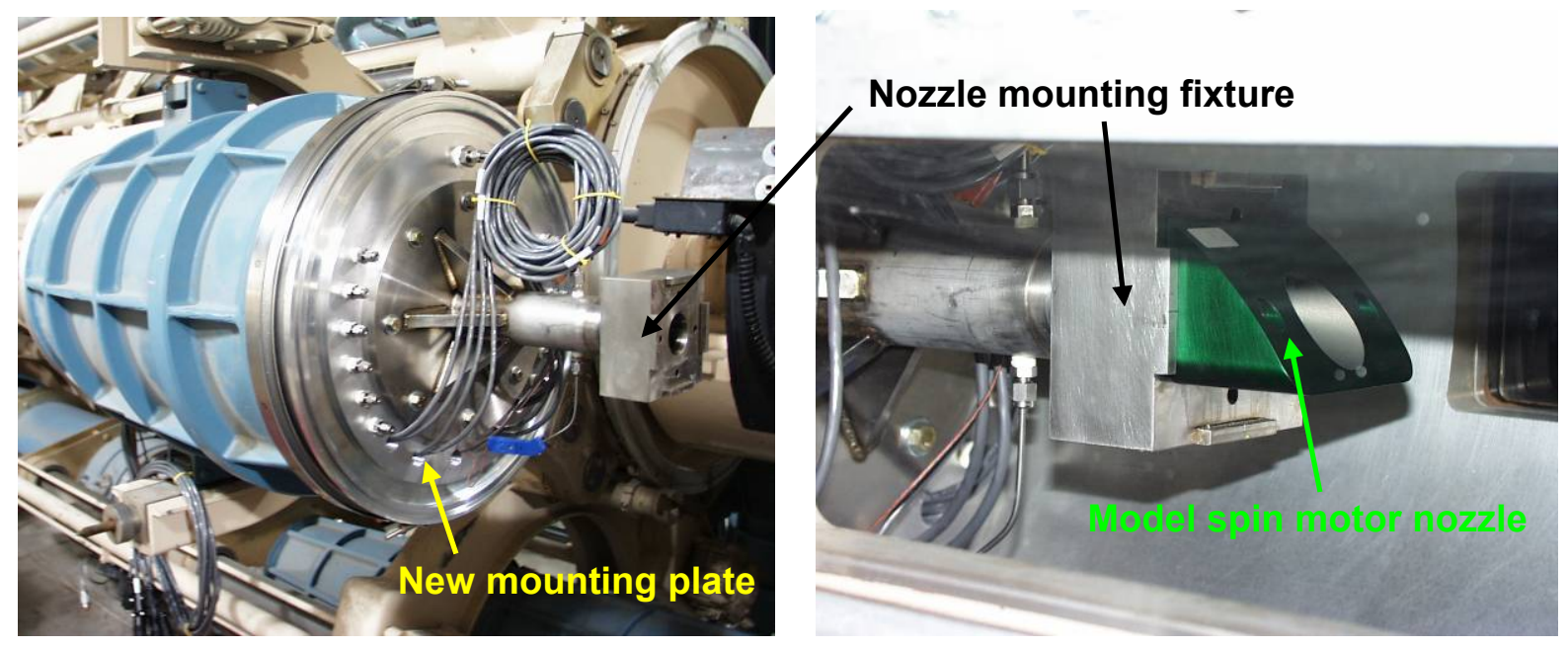

Figure 16. Nozzle flow characterization hardware during setup and in the $M=7.3$ test section.

heaters, heater cooling water pressure, and heater sonic orifices were disabled in this experiment. The only new ES\&H requirements were certification of the welds on the new hardware and a review of the new operating procedures. By modifying the existing $\mathrm{M}=7.3$ nozzle assembly and wind tunnel infrastructure instead of building a new facility, the cost of the nozzle characterization capability was made affordable. All wind tunnel modifications were designed, constructed, installed and ready to use in only four months.

\section{Nozzle Operational Stagnation Temperature}

Stagnation temperatures must be high enough to avoid condensation of the nitrogen gas as it expands from the stagnation chamber to the nozzle exit plane and the survey plane of the pitot probe. Condensation in either saturated or supersaturated form must be avoided because it introduces non-isentropic flow phenomena that prevent us from using pitot probe data to determine flow field Mach number and dynamic pressure. In the real spin motor nozzles, the temperature of the spin motor gas far exceeds that required to avoid condensation in the flight vehicle nozzle. In the full-scale wind tunnel experiments (where air was used in the nozzles), the stagnation temperature was maintained at $\sim 100{ }^{\circ} \mathrm{F}$, well above condensation limits. For the HWT nozzle flow characterization experiments, there was no convenient way to heat the nitrogen gas before it passed through the model spin motor nozzles. Therefore, we performed analyses to determine whether condensation would occur at the exit plane of each nozzle if we did not heat the nitrogen.

Daum and Gyarmathy ${ }^{10}$ present condensation onset static temperature data as a function of whether the nitrogen condenses at its saturation limit or at its supersaturation limit. At any given static pressure, the saturation temperature is the temperature at which quiescent nitrogen condenses due to spontaneous nucleation. However, nitrogen (and other gases as well) can remain in the gas phase below the quiescent saturation temperature if the gas can be expanded quickly, before the spontaneous nucleation process can cause condensation. By the time nucleation occurs, the gas has continued to expand to lower temperatures and pressures and is downstream of the location where quiescent condensation would have taken place. This phenomenon is called supersaturation and has often been observed in supersonic and hypersonic nozzles. The supersaturation temperature is lower than the saturation temperature 
Table 1. Minimum stagnation temperatures for avoiding nitrogen condensation at the nozzle exit for $P_{0}=1000 \mathrm{lbs} / \mathrm{in}^{2}$.

\begin{tabular}{|l|l|c|c|c|}
\hline \multirow{2}{*}{$\begin{array}{l}\text { Nozzle } \\
\text { Configuration }\end{array}$} & $\begin{array}{l}\text { Location in } \\
\text { Nozzle Exit } \\
\text { Plane }\end{array}$ & $\begin{array}{c}\text { Exit Mach } \\
\text { Number at } \\
\text { this Location }\end{array}$ & $\begin{array}{c}\text { Minimum } T_{0}\left({ }^{\circ} \mathrm{R}\right) \text { for } \\
\text { Saturated } \mathrm{N}_{2} \\
P_{0}=1000 \mathrm{lbs} / \mathrm{in}^{2}\end{array}$ & $\begin{array}{c}\text { Minimum } T_{0}\left({ }^{\circ} \mathrm{R}\right) \text { for } \\
\text { Supersaturated } \mathrm{N}_{2} @ \\
P_{0}=1000 \mathrm{lbs} / \mathrm{in}^{2}\end{array}$ \\
\hline \multirow{2}{*}{$\begin{array}{l}\text { Baseline Nozzle } \\
\text { and Nozzle } \\
\text { Configuration C }\end{array}$} & "Short" side & 3.30 & 450 & 420 \\
\cline { 2 - 5 } & Centerline & 3.45 & 470 & 440 \\
\cline { 2 - 5 } & "Long" side & 4.20 & 570 & 505 \\
\hline $\begin{array}{l}\text { Nozzle A: Scaled- } \\
\text { down Baseline }\end{array}$ & $\begin{array}{l}\text { Because all nozzle dimensions are scaled, the predicted stagnation } \\
\text { temperatures are the same as for the baseline nozzle at each exit location }\end{array}$ \\
\hline \multirow{2}{*}{$\begin{array}{l}\text { Nozzle } \\
\text { Configurations A, } \\
\text { B and D }\end{array}$} & "Short" side & 3.55 & 475 & 450 \\
\cline { 2 - 5 } & Centerline & 3.70 & 500 & 460 \\
\cline { 2 - 5 } & "Long" side & 4.50 & 610 & 545 \\
\hline
\end{tabular}

at any given static pressure. Daum and Gyarmathy's data show that the minimum temperature needed to avoid either saturation or supersaturation increases with the static pressure level at the nozzle exit plane.

Table 1 shows the minimum stagnation temperatures required to avoid saturation and supersaturation of the nitrogen at three locations along the exit plane of each model nozzle. The calculated saturation and supersaturation temperatures in Table 1 assume a nozzle stagnation pressure of $1000 \mathrm{lbs} / \mathrm{in}^{2}$ (the highest operational stagnation pressure), and therefore they represent the highest stagnation temperatures required for condensation-free operation of each nozzle. To calculate these "worst case" stagnation temperatures, the exit Mach number was determined from pitot pressure surveys at exit locations along the centerline and the "short" and "long" sides of each nozzle configuration. The exit static pressure is calculated at these Mach numbers and Daum and Gyarmathy's data were used with the calculated static pressure to estimate the static temperature at which either saturation or supersaturation will occur. Using the 1-D steady isentropic flow equations ${ }^{11}$, those static temperature condensation limits were converted into the minimum stagnation temperatures for operating at either saturation or supersaturation limits.

Table 1 indicates that we must heat the nitrogen to approximately $570^{\circ} \mathrm{R}$ in order to avoid saturation in the exit plane of the baseline nozzle and nozzle Configurations $\mathrm{A}$ and $\mathrm{C}$ when operating them at $1000 \mathrm{lbs} / \mathrm{in}^{2}$. Because Nozzle Configurations B and D have higher exit Mach numbers, we must heat the nitrogen to approximately $610^{\circ} \mathrm{R}$ in order to avoid saturation in the exit plane of those nozzles. Table 1 also shows that, if Daum and Gyarmathy's supersaturation data applies to these nozzles, we would need a stagnation temperature of at least $505^{\circ} \mathrm{R}$ for condensation-free operation of the baseline nozzle and nozzle Configurations $\mathrm{A}$ and $\mathrm{C}$, and we would need to operate nozzle Configurations B and D at $545^{\circ} \mathrm{R}$ or higher.

The analysis was expanded to calculate the condensation limits for several exit flow Mach numbers as a function of nozzle stagnation pressure. Figure 17 shows the variation of minimum required nozzle stagnation temperature with nozzle stagnation pressure at three representative exit flow Mach numbers. Also shown on Fig. 17 is the operational range of stagnation temperature produced in the nozzle characterization experiment when the nitrogen 
gas is not heated. Note that this operational range is sufficient to avoid saturation on the baseline nozzle centerline (where $M=$ $\sim 3.45$ ) at all stagnation pressure levels. However, the minimum stagnation temperature required to avoid condensation on the "long" side of the baseline nozzle (where $M=\sim 4.2$ ) cannot be achieved unless the operational stagnation pressure is set below $400 \mathrm{lbs} / \mathrm{in}^{2}$.

Basing the condensation limit of the model spin motor nozzles upon the saturation temperature of quiescent nitrogen may result in calculated minimum stagnation temperatures that are too high because the small nozzle dimensions and rapid expansion of the flow in these nozzles should promote supersaturation. As noted earlier, the HWT $\mathrm{M}=8$ and 14 nozzles use the same nitrogen gas supply as the model spin motor nozzles. We observed levels of supersaturation in the HWT $M=8$ and 14 nozzles $^{12}$ that occur at lower temperatures than those predicted by Daum and Gyarmathy. Since the HWT nozzles and model spin motor nozzles have comparable rates of expansion, we would expect the level of supersaturation in the model nozzles to be comparable to the level observed in the HWT nozzles.

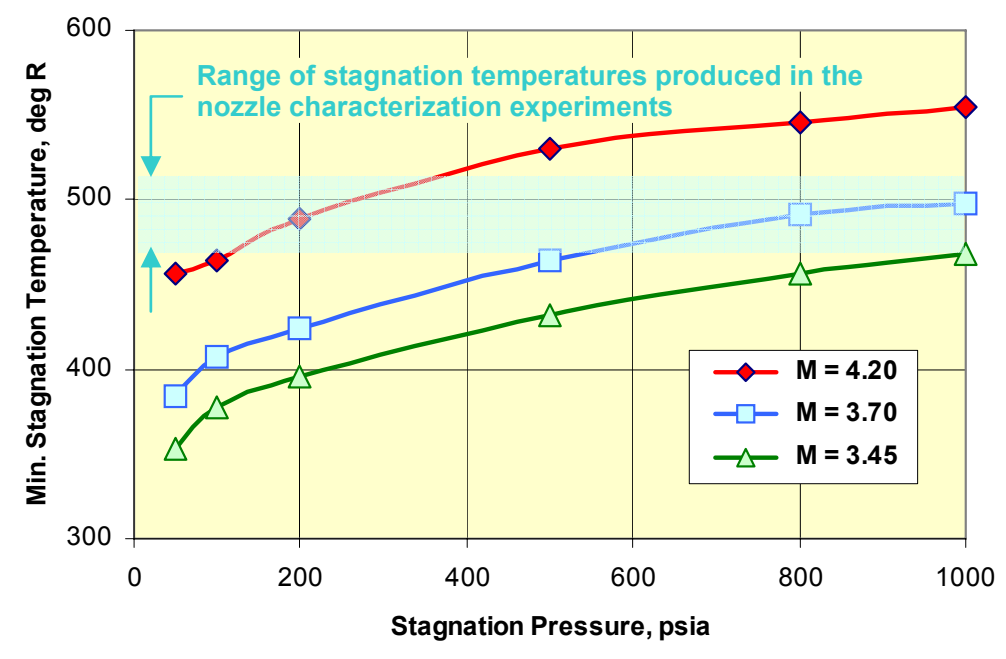

Figure 17. Variation of minimum stagnation temperature required to avoid nitrogen gas saturation in the model spin motor nozzles.

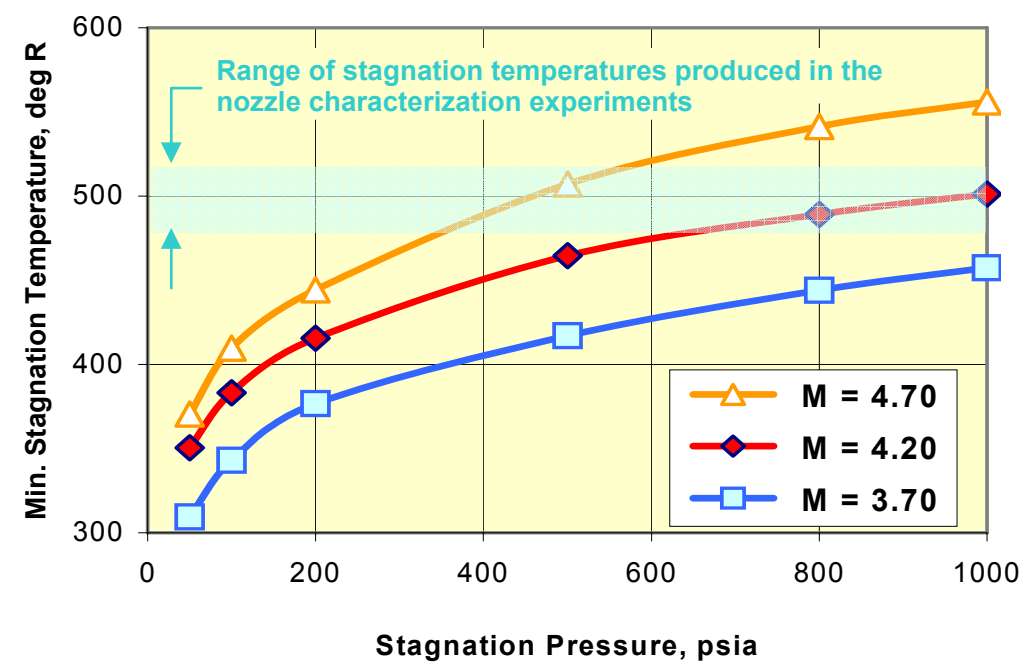

Figure 18. Variation of minimum stagnation temperature required to avoid nitrogen gas supersaturation in the model spin motor nozzles.

Figure 18 presents the minimum stagnation temperatures required to avoid nitrogen gas supersaturation in the model spin motor nozzles, assuming that Daum and Gyarmathy's data applies. The figure indicates that we may encounter supersaturated flow at stagnation pressures above $\sim 600 \mathrm{lbs} / \mathrm{in}^{2}$ at the exit plane on the "long" side of the baseline and Configuration $\mathrm{A}$ and $\mathrm{C}$ nozzles, where the highest Mach number is 4.2. For Configuration $\mathrm{B}$ and $\mathrm{D}$ nozzles, Figure 18 indicates that the supersaturation limit could be reached at $P_{0}=\sim 400$ $\mathrm{lbs} / \mathrm{in}^{2}$, where the highest exit plane Mach number is $\sim 4.5$. For stagnation pressures below $\sim 350 \mathrm{lbs} / \mathrm{in}^{2}$, supersaturation effects should not be observed for Mach numbers as high as 4.7. 
Because our analysis indicated that supersaturation of the nitrogen could occur in regions of high Mach number in the nozzle exit flow, we evaluated several ways to heat the nitrogen. The temperature of the nitrogen gas supplied to the HWT by the current $\mathrm{M}=8 / 14$ nitrogen supply system ranged between $472^{\circ} \mathrm{R}$ (after several successive runs using high mass flow rates) to $515^{\circ} \mathrm{R}$ (at the end of runs using low mass flow rates). There is no way to heat the storage bottles, and it would have been prohibitively expensive and time-consuming to heat the preheater and the pipes that bring the nitrogen from the storage tanks to the HWT. Therefore, we decided to operate all model nozzles with unheated nitrogen. We determined the extent of condensation in each nozzle by comparing Mach number surveys at the same location over the range of stagnation pressures between 62 and $1000 \mathrm{lbs} / \mathrm{in}^{2}$. We would expect the surveys to be very similar across the entire range of stagnation pressure, unless non-isentropic effects caused by condensation altered the measured pitot pressures as stagnation pressure was increased.

Figure 19 presents the centerline $(Y=0)$ Mach number surveys measured 0.010 inches downstream of the exit of Configuration C Nozzle 40 (C-40) for stagnation pressures ranging from 64 to $950 \mathrm{lbs} / \mathrm{in}^{2}$. The stagnation temperatures for these surveys ranged between 490 and $506^{\circ} \mathrm{R}$. Figure 17 indicates that we should not observe any saturation of the nitrogen gas at the centerline of the nozzle, where the Mach number is $\sim 3.45$. Figure 19 shows only minor variations in Mach number with stagnation pressure around the centerline. The same features of the wave structure propagating through the flow are observed in each survey, independent of $P_{0}$. The only observed effects of increasing the stagnation pressure were related to the thinning of the nozzle wall boundary layer (Reynolds number increases in proportion to $P_{0}$

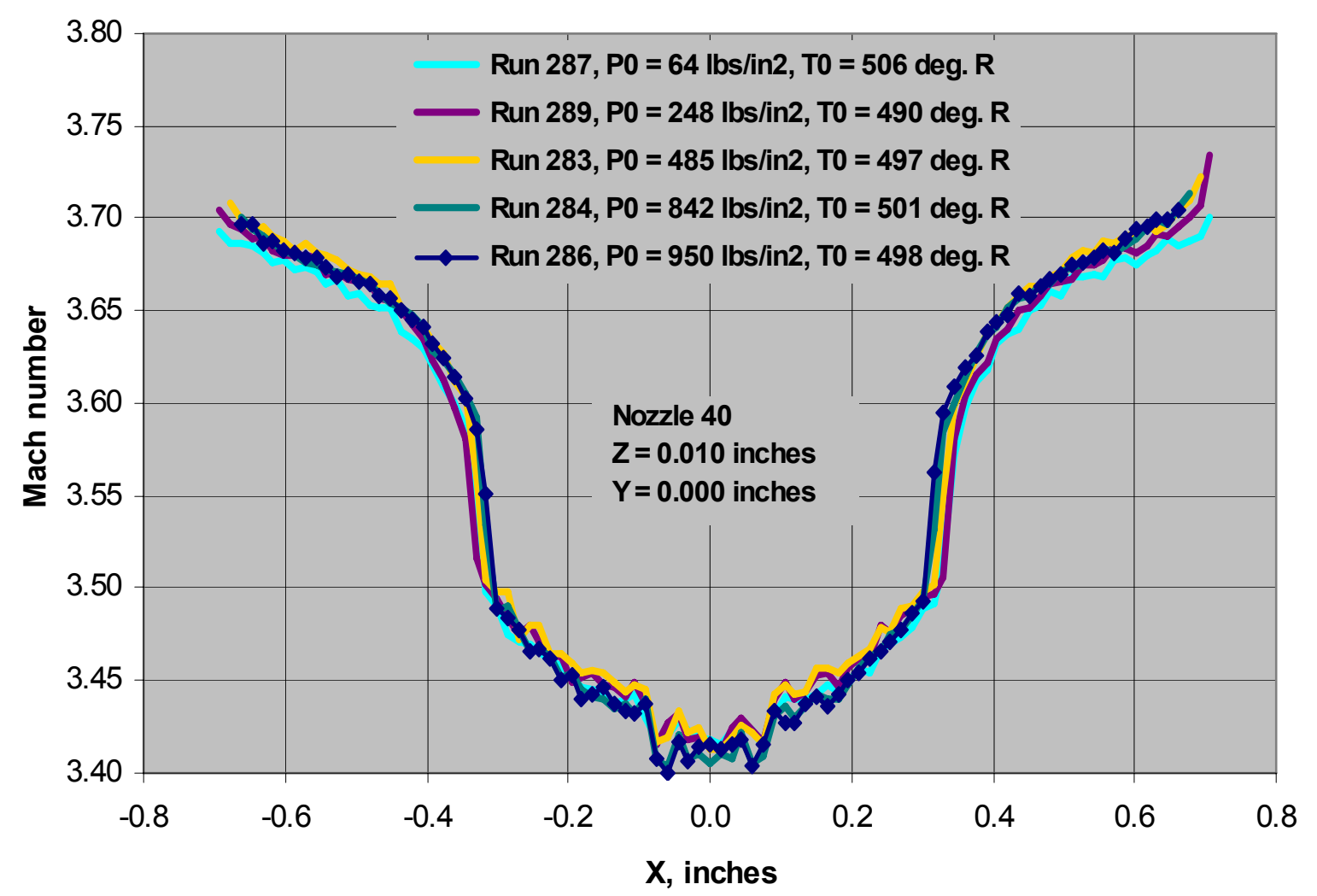

Figure 19. Centerline Mach number surveys for Nozzle $C-40$ for $Z=0.010$ inches and $64 \leq P_{0} \leq 950 \mathrm{lbs} / \mathrm{in}^{2}$. 
and the nozzle wall boundary layer thickness decreases with increasing Reynolds number). If condensation were present, non-isentropic phenomena would cause abrupt shifts in the Mach number profiles. Because the profiles are so similar, we conclude that there is no nitrogen condensation in this region of the flow. Furthermore, the profiles in Figure 19 retain their similarity out to the edge of the nozzle flow where the Mach number is $\sim 3.7$. Figure 17 indicates that we should not expect to experience any saturation of the nitrogen gas up to $M=$ 3.7 at $T_{0}=500^{\circ} \mathrm{R}$, even for $P_{0}=950 \mathrm{lbs} / \mathrm{in}^{2}$. This prediction from Daum and Gyarmathy's data appears to be borne out by the similarity of the Mach number profiles. From Figures 17 and 19, we conclude that our range of operating stagnation temperatures is adequate to avoid any condensation effects up to $M=3.7$.

To determine whether the nozzle flow is free from condensation at Mach numbers above 3.7, surveys were made for the flow produced by Configuration D Nozzle 30 (D-30). This nozzle has the same exit dimensions as Nozzle C-40, but it has a smaller throat. Therefore, the exit Mach number is higher for Nozzle D-30 due to its higher area ratio. Centerline surveys were made at $Z=0.010$ inches downstream of the nozzle exit for $62 \leq P_{0} \leq 980 \mathrm{lbs} / \mathrm{in}^{2}$ (Figure 20a) and $Z=0.460$ inches for $64 \leq P_{0} \leq 481 \mathrm{lbs} /$ in $^{2}$ (Figure 20b). Although there is more scatter in these surveys than there was in the Nozzle C-40 surveys (Fig. 19), the similarity of the Mach number profiles suggests that there is negligible condensation up to $M=4.0$ for stagnation pressures up to $980 \mathrm{lbs} / \mathrm{in}^{2}$ and up to $M=4.4$ for stagnation pressures up to 481 $\mathrm{lbs} / \mathrm{in}^{2}$ at our operational stagnation temperatures. The model spin motor nozzles appear to benefit from a delay in the spontaneous nucleation process that results in supersaturation. Perhaps the delay is due to the absence of nucleation sites in the pure nitrogen (less than 4 parts per million of $\mathrm{O}_{2}$ ) used in these experiments.

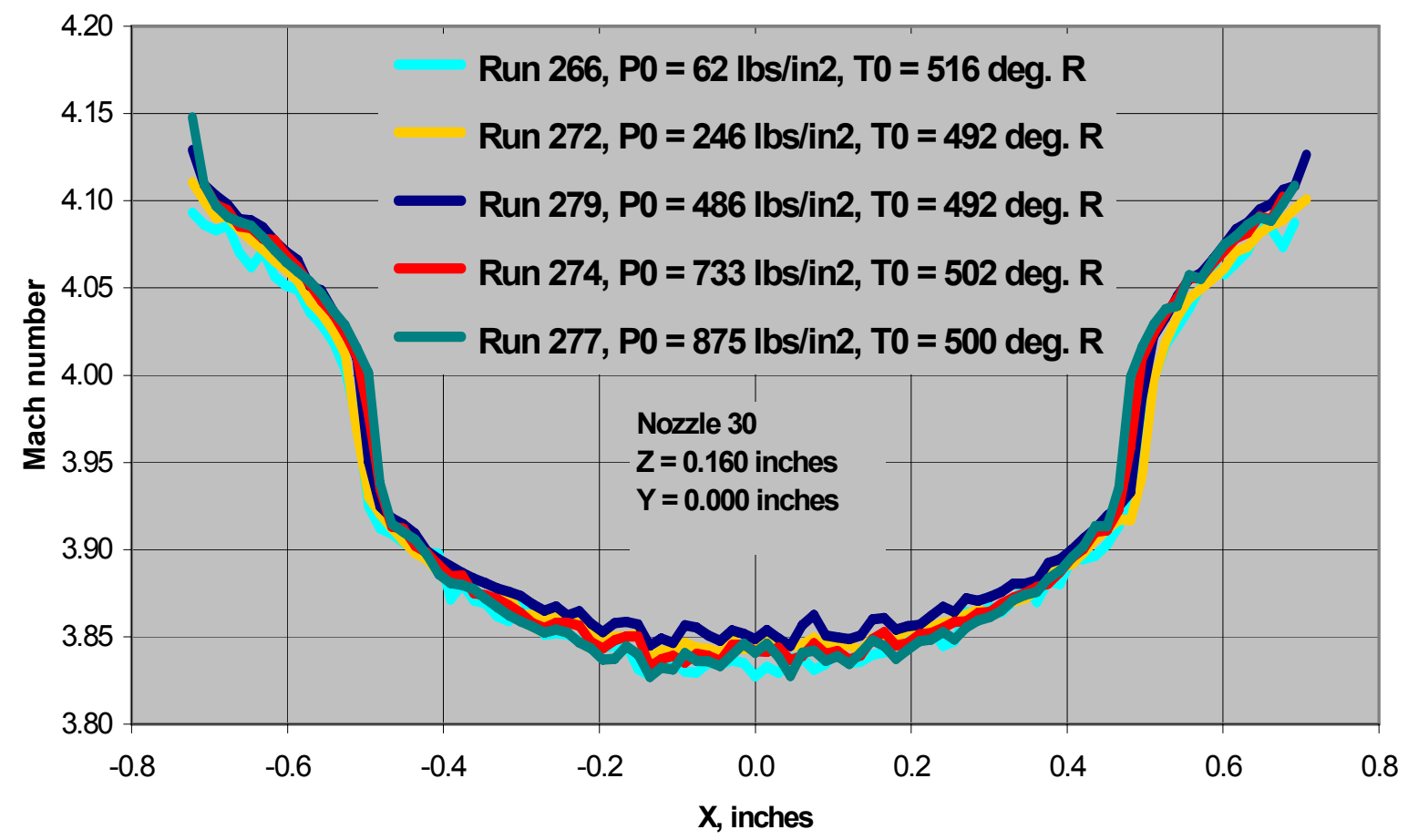

Figure 20a. Centerline Mach number surveys for Nozzle D-30 for $Z=0.010$ inches and $62 \leq P_{0} \leq 980 \mathrm{lbs} / \mathrm{in}^{2}$. 


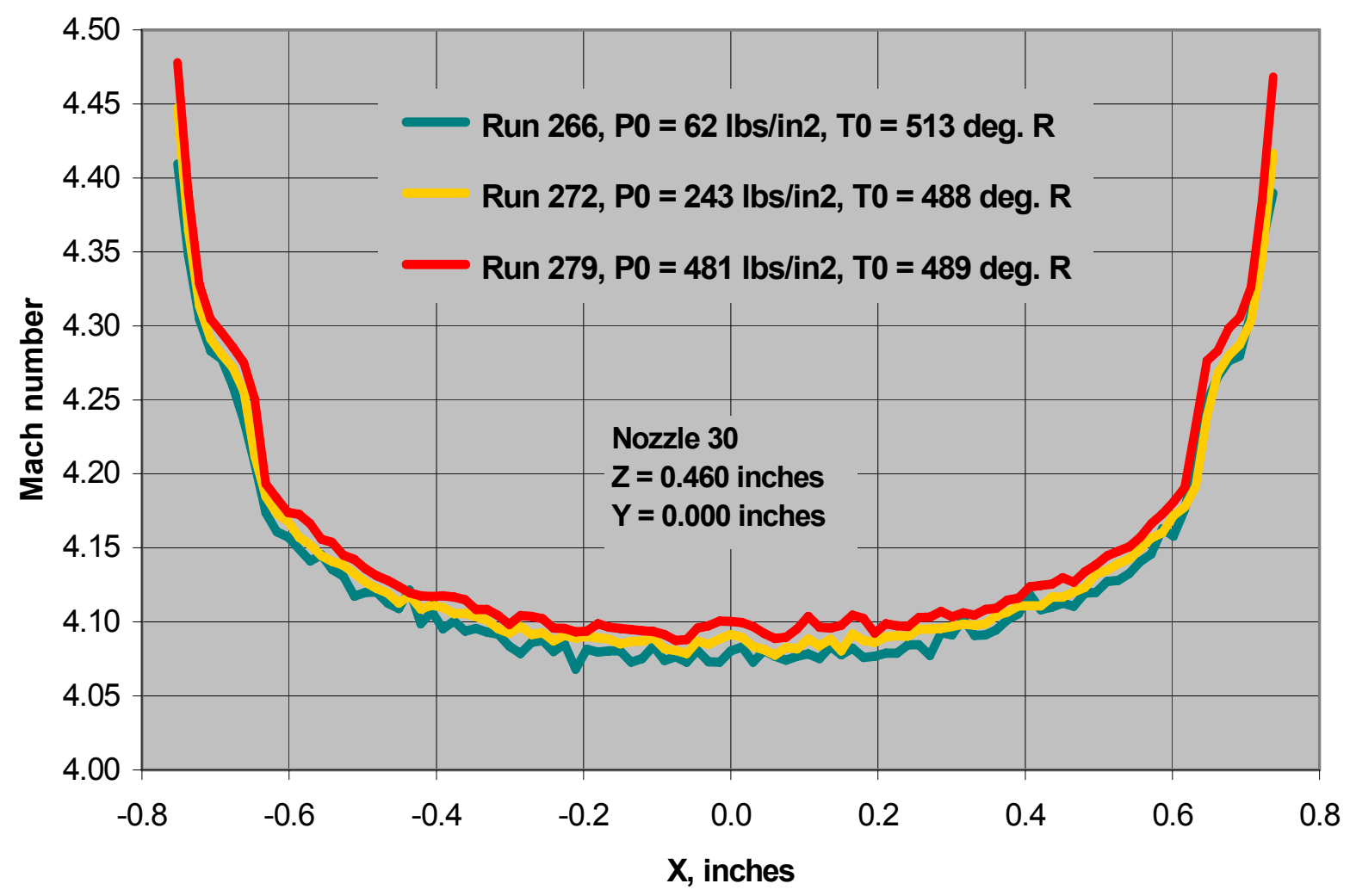

Figure 20b. Centerline Mach number surveys for Nozzle D-30 for $Z=0.460$ inches and $62 \leq P_{0} \leq 481 \mathrm{lbs} / \mathrm{in}^{2}$. 


\section{Pitot Probe Measurements}

Pitot probes were used to survey the flow emanating from each nozzle at nozzle stagnation pressures between 60 and $1000 \mathrm{lbs} / \mathrm{in}^{2}$, the same pressure range that was used in the full-scale wind tunnel experiments. The pitot probes used in the model nozzle characterization measurements are made from flat-tipped (no bevel) 1/16-inch-diameter stainless steel tube welded into 1/8-inch-diameter stainless steel tubing. The inner diameter of the 1/16-inch-OD tubing was 0.022 inches.

Figure 14 shows the probe holder which positions the pitot probe tips at the nozzle exit plane. The probe holder can accommodate up to five probes spaced 0.500 inches apart. However, to avoid any shock interference from adjacent probes, we used no more than three probes at one time. For the axial (traverse in $Z$ with $X=Y=0$ ) pitot traverses and radial surveys (traverse in $X$ at $Y=0$ and specified $Z$ locations) made in this experiment, a single probe was mounted in the center probe holder. For the surveys conducted at nonzero $Y$ values, three probes were mounted in the probe holder, positioned as shown in Figure 10. The probe holder assembly could be adjusted manually to acquire data at additional vertical positions above/below the $Y=0$ plane.

Automation Concepts Inc. (ACI) developed a four axis motion control system using a Parker Compumotor 6000 series stepper motor controller and linear position stages. A two-axis traverse drive was mounted on the bottom of the test section. One drive axis traversed the probes across the test section and the other traversed the probe along the flow axis. The probe drive was mounted inside the test section on the bottom test section window, as shown in Figure 14. The rigid traverse assembly did not flex under the influence of aerodynamic drag. Probe drives and controllers could repeat $X$ and $Z$ positions to within \pm 0.001 inches.

ACI fabricated an interface panel with associated connectors and cables to operate the fouraxis stepper motor traverse system. ACI's LabView program controlled the system using an Ethernet interface which had the capability to move the traverse continuously in an arc with predefined coordinates of the arc center and radius. However, this program made no provisions for acquiring or synchronizing pressure data during the continuous positional moves. Therefore, we fabricated a data patch panel to interface the pressure transducers, thermocouple, power supplies, terminal blocks and A/D board. A LabView data acquisition program, which was written using the motion interface drivers provided from ACI, acquired pressure (stagnation chamber pressure, ambient pressure in the test section, and pressures measured by the pitot probes) and temperature (using a type $T$ thermocouple installed in the stagnation chamber) data while controlling the motion of the 2-axis traverse system. Another LabView program was written to reduce the recorded data to engineering units; pressure (lbs/in ${ }^{2}$ absolute), Mach number, temperature (deg. F) and position (inches). Microsoft Excel was used to plot the trends and profile of the data, i.e., nozzle Mach numbers vs. probe tip lateral survey location at several axial locations.

The traverse was "homed" (moved to home position) prior to a probe tip position calibration. An alignment block was installed in place of the nozzle to calibrate the position of the probe(s) in the vertical and lateral directions. The vertical position was adjusted manually; the lateral and axial adjustments were made with the traverse system. Since each nozzle was slightly different, the axial position was determined by iterating on small axial movements with careful feeler gage measurements between the probe tip and the nozzle contour after positioning the probe laterally 0.040 inches outside the nozzle exit plane. This measurement 
was verified again on the opposite side, also 0.040 inches from the exit plane. To protect the probe tip(s) from possible contact with the nozzle, a clearance of 0.010 inches from the nozzle contour was typically used for the closest lateral sweep. The lateral sweeps usually extended beyond the nozzle wall exit plane by at least 0.015 inches. Offset values were determined from periodic position calibrations so that the data could be reduced to actual coordinates relative to the nozzle center-line exit plane.

The digitally-controlled probe drives were programmed to move the probes in the axial direction (along the $Z$ axis) and lateral direction (along the $X$ axis, perpendicular to the page in Figure 14) during the run. After the test conditions were stabilized, the traverse/data sequence was initiated. A data sweep actually was composed of iterations of a small linear move, a pause, and then a data acquisition scan on all measured channels. All data were acquired when the probe was stationary after the pause of $75 \mathrm{~ms}$. Because the pressure transducers were located outside the tunnel only a few feet away from the probes, the 75msec dwell time at each survey position was sufficient to assure that the measurement represented a steady state value. Data were averaged over $1.67 \mathrm{msec}$ after the pause. To facilitate their calibration, each pressure transducer could be switched to a common pressure manifold without disturbing the connections to the probes.

Omega model PX01 pressure transducers with pressure ranges of 0 to 300, 500 and 1000 lbs/in ${ }^{2}$ gage (psig) were used for measuring the nozzle stagnation pressure. We used the transducer having the lowest acceptable range to accommodate the stagnation pressure for that run. The pitot probe measurements utilized the Setra model 205-2. With an assortment of pressure transducers, we acquired data on up to three probes at a time using a $25 \mathrm{lb} / \mathrm{in}^{2}$ absolute (psia), 50 psig, 100 psig or 250 psig range that was selected to best fit the level of pitot pressure being measured. Gage pressures were converted to absolute pressures using a Druck model DPI 140 digital barometer. The ambient pressure in the test section was measured with a Setra model 205-2 15 psia pressure transducer. Schlieren photographs showed that the nozzles were under-expanded throughout the run; therefore, ambient pressure was never high enough to cause nozzle flow separation.

Mach number distributions in the inviscid flow across the nozzle exit plane were determined from simultaneous measurements of nozzle stagnation pressure $P_{0}$ and pitot pressure $P_{t}$ in conjunction with the Rayleigh pitot formula ${ }^{7}$, given below for air and nitrogen $(\gamma=1.4)$ :

$$
P_{t} / P_{0}=\left[6 M^{2} /\left(M^{2}+5\right)\right]^{3.5} *\left[6 /\left(7 M^{2}-1\right)\right]^{2.5}
$$

A simple Newton-Raphson iteration scheme was used with Eq. 2 to obtain the correct Mach number $M$ from the measured value of $P_{t} / P_{0}$. Starting with an initial value of $M=3.0$, the measured value of $P_{t} / P_{0}$ and the value computed from Eq. 2. converged to within $10^{-6}$ in fewer than six iterations. The assumptions underlying the Rayleigh pitot probe formula are exactly met for pitot measurements in the inviscid flow field of each nozzle.

Mach number, rather than pitot pressure ratio, is used throughout this report to characterize the nozzles. Once the Mach number is known, all other properties of the inviscid nozzle flow can be calculated from one-dimensional gas dynamics equations ${ }^{7}$. For example, centerline exit dynamic pressure $Q_{\text {nozzle }}$ is determined by using known values of centerline exit Mach number $M_{n o z}$ and stagnation pressure in Eq. 3:

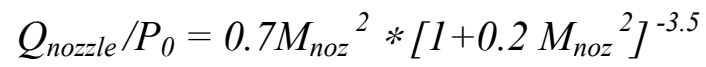




\section{Measurement Error Sources and Accuracy}

\section{Pressure Instrumentation Errors}

By using transducers whose maximum pressure ratings were matched to the levels of $P_{0}$ and $P_{t}$ measured for each run, we were able to minimize instrumentation errors of pitot and stagnation pressure transducers. Stated accuracy of the Omega transducers was $\pm 0.05 \%$ of maximum rated pressure; for the Setra transducers, the stated accuracy was $\pm 0.11 \%$ of maximum rated pressure. By choosing among three transducers for $P_{0}$ and four transducers for $P_{t}$, the maximum root mean square instrumentation error for $P_{t} / P_{0}$ was always less than $0.4 \%$ of the measured values. Daily in situ transducer calibration checks showed negligible zero shift or slope change.

\section{Repeatability}

Following the methodology of Oberkampf, et al. ${ }^{13,14}$, we attempted to assess the uncertainty in experimental measurements of Mach number by examining the symmetry and repeatability of survey data. For nozzles C-40, C-41 and D-31, surveys using a single pitot probe were repeated at the same $Z$ locations and stagnation pressure to assess the repeatability of the Mach number deduced from Eq. 2. Repeat runs along the $Y=0$ plane were made at axial stations of $0.010,0.160,0.310,0.460,0.610$ and 0.760 inches downstream of the nozzle. Each survey consisted of 90-100 points in the $X$ direction, spaced 0.015 inches apart. Between repeated runs, the probe was driven to a "rest" position two inches downstream of the nozzle exit. For the repeat run, the probe drive was commanded to position the probe at the same $Z$ values that were surveyed in the first run. Neither the nozzle nor the probe within the probe holder were moved between surveys, so the only spatial error introduced in these paired comparisons was the error in the ability of the digital probe drive to return to the same $Z$ values. Bias errors (such as pressure transducer errors, probe/nozzle alignment errors and uncertainty in the absolute location of the probe tip relative to the nozzle) were repeated in these surveys and therefore cannot be detected. Repeat surveys give an indication of the random errors in the pressure measurement and probe drive positioning.

Figure 21 compares the Mach numbers measured in repeated surveys made in Nozzle C-40 at $P_{0}=250 \mathrm{lbs} / \mathrm{in}^{2}$. For each repeated survey, the difference in Mach number between surveys was calculated at each measurement point, and the standard deviation of the differences was calculated for that survey. A total of 36 repeat surveys at different values of $P_{0}$ and $Z$ were made for nozzles C-40, C-41 and D-30. Table 2 shows that the average standard deviation in Mach number depended somewhat on the nozzle being probed; it ranged from 0.00270 for Nozzle D-30 to 0.00464 for Nozzle C-41. Considering all 36 surveys as a

Table 2. Average standard deviation in Mach number for repeated surveys and symmetry comparisons.

\begin{tabular}{lccc}
\hline Comparison & $\begin{array}{c}\text { Nozzle } \\
\text { Config.-No. }\end{array}$ & $\begin{array}{c}\text { No. of } \\
\text { surveys } \\
\text { averaged }\end{array}$ & $\begin{array}{c}\text { Avg. Std. } \\
\text { Deviation } \\
\text { in } \boldsymbol{M}\end{array}$ \\
\hline Repeatability: & D-30 & 12 & 0.00270 \\
& D-31 & 0 & -- \\
& C-40 & 18 & 0.00311 \\
& C-41 & 6 & 0.00464 \\
Symmetry: & all & 36 & 0.00323 \\
0-degree/90-degree & D-31 & 4 & 0.00406 \\
0-degree/180-degree & D-30 & 12 & 0.00513 \\
& D-31 & 4 & 0.00451 \\
& C-40 & 7 & 0.00852 \\
& C-41 & 6 & 0.00736
\end{tabular}




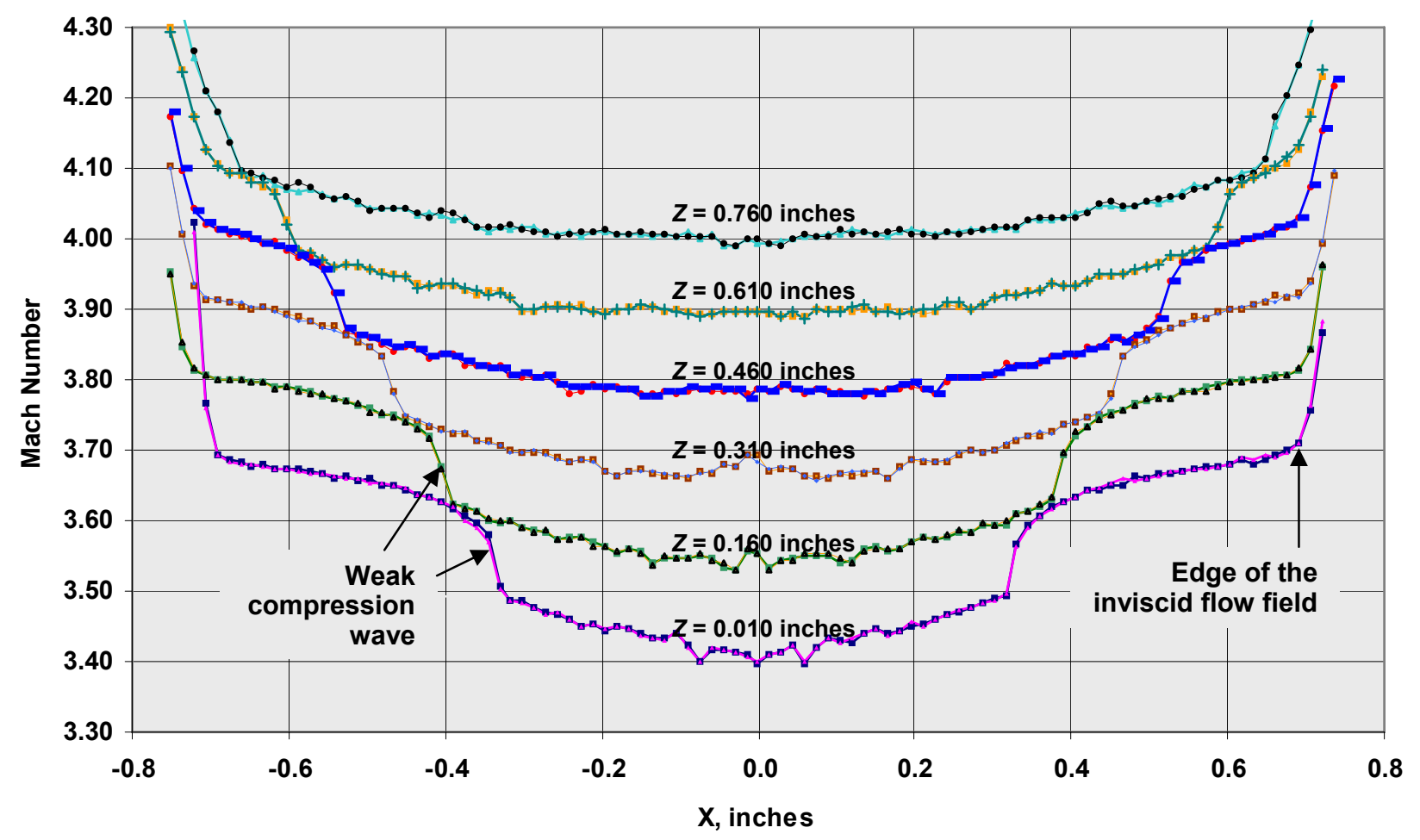

Figure 21. Repeated surveys on the $Y=0$ plane at six axial locations downstream of Nozzle C-40, $P_{0}=250 \mathrm{lbs} / \mathrm{in}^{2}$.

whole, the average standard deviation in Mach number was 0.00323 . Using Eq. 3, we can show that an error of 0.00323 in Mach number due to the uncertainty indicated by this repeatability data will cause less than a $0.3 \%$ error in $Q_{\text {nozzle }}$ when the nominal Mach number is 3.5 .

Note that, in Figure 21 and in all other surveys, the Mach number appears to increase rapidly at the outer boundaries of each survey. This is not a real physical phenomenon; it is instead an artifact of our data reduction assumptions. When the probe tip enters the shear layer (the continuation of the nozzle wall boundary layer), viscous effects cause both the measured pitot pressure and the local stagnation pressure to decrease. Because the data reduction method assumes that the stagnation pressure is constant (equal to the nozzle stagnation pressure) at all survey points, it interprets a decrease in pitot pressure to be caused by an increase in Mach number, in accordance with Eq. 2. Therefore, the $X$ values where Mach number begins to increase rapidly should be interpreted as the location of the outer boundary of the inviscid nozzle flow, and no attempt should be made to compare experimentally determined Mach number to inviscid computational results beyond these $X$ values.

\section{Probe Position Errors}

As noted previously, the nozzle was removed and replaced by a special fixture to locate the probe tip in the test section. Using the fixture, the probe tip location with respect to the fixture could be determined to within \pm 0.002 inches. To determine the position of the probe tips relative to the true $Z=0$ location for each nozzle, shims were used to measure the distance between the probe tip and the left and right faces of the nozzle. Using this process, the uncertainty of the probe tip location along the $Z$ axis was estimated to be \pm 0.007 inches. 
Axial surveys along the nozzle centerline $(X=Y=0)$ showed that the nozzle flow continues to expand downstream of the nozzle exit plane (see Figure 21, for example). At the exit plane, the Mach number gradient is approximately 1 per inch. This means that the uncertainty in axial probe tip location of \pm 0.007 inches can cause an error in Mach number of approximately \pm 0.007 .

The excellent symmetry of the nozzle flows (see below) was used to estimate the error in probe tip location along the $X$ and $Y$ axes. The uncertainty in probe tip location along each of these axes was estimated to be approximately \pm 0.010 inches. Because the Mach number gradient along these axes was observed to be very small (except in the weak compression wave), the corresponding error in Mach number due to this probe location uncertainty is less than the Mach number uncertainty along the $Z$ axis. We estimate a Mach number uncertainty of \pm 0.005 along the $X$ and $Y$ axes due to probe position uncertainty. Again using Eq. 3, an error of 0.005 in Mach number due to the uncertainty in axial probe location will cause a $0.43 \%$ error in $Q_{\text {nozzle }}$ when the nominal Mach number is 3.5 .

\section{Flow symmetry surveys}

Figure 22 compares $P_{0}=500 \mathrm{lbs} / \mathrm{in}^{2}$ survey results using a single pitot probe for Nozzle D-31 at four axial locations downstream of the nozzle exit. At each axial location, one survey was made along the $Y=0$ axis and the other survey was made along the $X=0$ axis in order to compare the 0-degree/90-degree symmetry of the flow. Surveys along the $X=0$ axis were made by rotating the nozzle by 90 degrees in the nozzle mounting fixture. The probe drive settings for $X=Y=Z=0$ were kept the same in each survey pair.

$X=0$ surveys were symmetric for $-0.5 \leq Y \leq+0.5$ inches but were not symmetric outside of \pm 0.5 inches due to the intrusion of the expansion fan generated at the exit on the "short side" of the scarfed nozzles. Within the region of symmetry, the flow does not know that the nozzles are scarfed. A point-to-point comparison of the $X=0$ surveys in this region to the

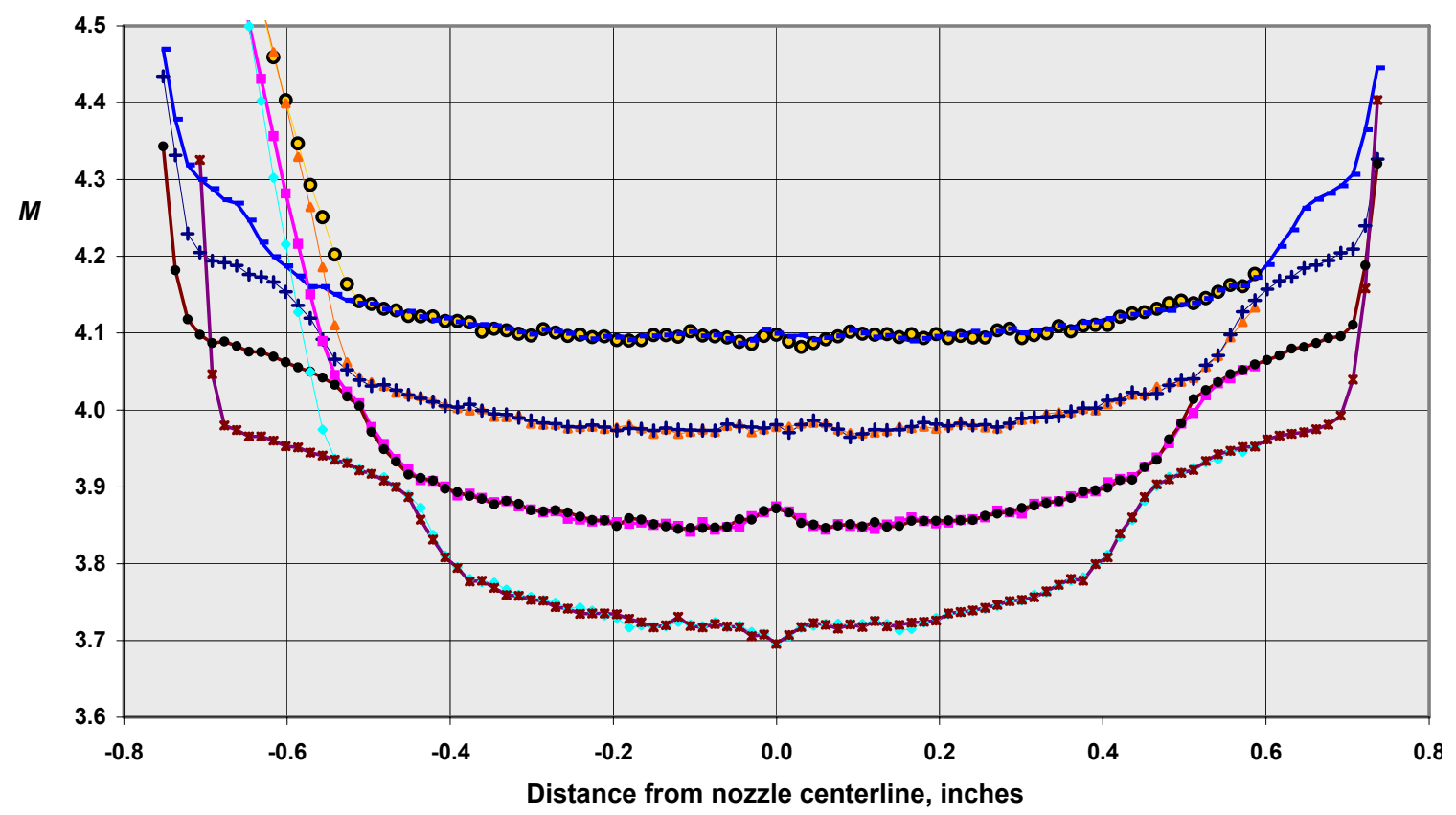

Figure 22. Comparison of Nozzle D-31 Mach number surveys along the $X=0$ and $Y=0$ axes for (bottom to top) $Z=0.010,0.160,0.310$ and 0.460 in., $P_{0}=500 \mathrm{lbs} / \mathrm{in}^{2}$. 
$Y=0$ surveys for $-0.5 \leq X \leq+0.5$ resulted in an average standard deviation in Mach number of 0.00406 , only slightly larger than the average standard deviation in Mach number for repeat runs.

0 -degree/180-degree flow symmetry was assessed by comparing the Mach numbers at survey points on the $Y=0$ plane at equidistant but opposite sides of the nozzle centerline. Table 2 shows that the average standard deviation in Mach number due to flow asymmetries was between 1.6 and 2.7 times the repeat run values, depending upon the nozzle being surveyed. These data indicate that the asymmetries are real and measurable, but quite small. The largest average standard deviation in Mach number due to nozzle asymmetry was 0.00852 for Nozzle C-40. This value is only $0.24 \%$ of the centerline Mach number at the nozzle exit. An error of 0.00852 in Mach number will cause a $0.7 \%$ error in $Q_{\text {nozzle }}$ when the nominal Mach number is 3.5 .

\section{Probe angle effects}

Depending upon the flow conditions and probe geometry, pitot pressure measurements may have to be corrected for errors caused by the disturbance that the probe makes in the flow field into which it has been inserted. Chue ${ }^{15}$ provides a comprehensive review of the physical phenomena that affect pitot probe accuracy. For our nozzle flow measurement environment, Chue's results indicate that probe tip geometry errors are negligible. However, we did attempt to quantify the errors caused by the angle of the flow relative to the probe orientation.

In the surveys reported here, the probe was aligned with the centerline axis of the nozzle and was swept across the nozzle exit plane. Because the flow is expanding, it is aligned with the probe only on the nozzle centerline; at radial locations off of the centerline, the flow is at an unknown angle with respect to the probe. Near the nozzle wall, the flow angle relative to the nozzle centerline approaches the nozzle expansion half-angle (15 degrees). Data given in Chue's monograph indicate that the reduction in pitot pressure caused by probe angle is small (but observable) for angles below 10 degrees, but at an angle of 15 degrees the measured value may be $\sim 5 \%$ below the correct value, resulting in a $1.7 \%$ error in centerline exit Mach number if the nominal Mach number is 3.7.

To assess the magnitude of measurement errors due to probe angle effects, surveys in the $Y=$ 0 plane were made with the probe intentionally canted at 5 degrees in the plane of the survey. Twenty-four of these comparative surveys were made for Nozzle C-40 and twenty-five were made for Nozzle D-30. Figure 23 compares pitot pressure ratio $\left(P_{t} / P_{0}\right)$ data from one of the surveys for Nozzle D-30. On the centerline of the nozzle, the flow is aligned with the 0 -degree probe, so any difference in the centerline pitot measurements is due solely to the 5degree probe cant angle. Using a cubic polynomial fit of centerline pitot survey data from all of the Nozzle D-30 comparison surveys, a $0.5 \%$ reduction in $P_{t} / P_{0}$ due to the 5 -degree probe angle was observed. The average reduction measured in Nozzle C-40 surveys was $0.1 \%$.

Unfortunately, bias errors in probe tip location (in both $Z$ and $X$ directions) between the two surveys in the comparison produced differences in centerline pitot pressure that were comparable to the expected differences caused by a 5-degree probe angle. We conclude that probe position errors obscure the effects of probe angle in these rapidly expanding nozzle flows and invalidate our in situ method of obtaining quantitative measurements of probe angle effects on pitot pressure error by comparing centerline measurements from 0 - and 5degree probes. 
Nevertheless, the comparative survey results can be used to estimate the magnitude of the flow angle in the nozzles. As the canted probe is traversed in the negative $X$ direction, the local flow angle is added to the 5-degree probe cant angle. Figure 23 shows that this results in significant measurable differences in the values of $P_{t} / P_{0}$ acquired by the two probes beyond $X \cong-0.3$ inches. As the probe is traversed in the positive $X$ direction, the local flow angle in the nozzle is subtracted from the 5-degree probe cant angle and therefore the difference in $P_{t} / P_{0}$ between the two surveys decreases. Figure 23 indicates that the two probes give approximately the same value of $P_{t} / P_{0}$ at $X \cong+0.5$ inches. Measuring the same value of $P_{t} / P_{0}$ can only occur when the flow angle with respect to the probe tip is the same for the 5 -degree probe as it is for the 0 -degree probe. This can only be the case if the flow angle is directed outward from the nozzle centerline by 2.5 degrees at $X \cong+0.5$ inches.

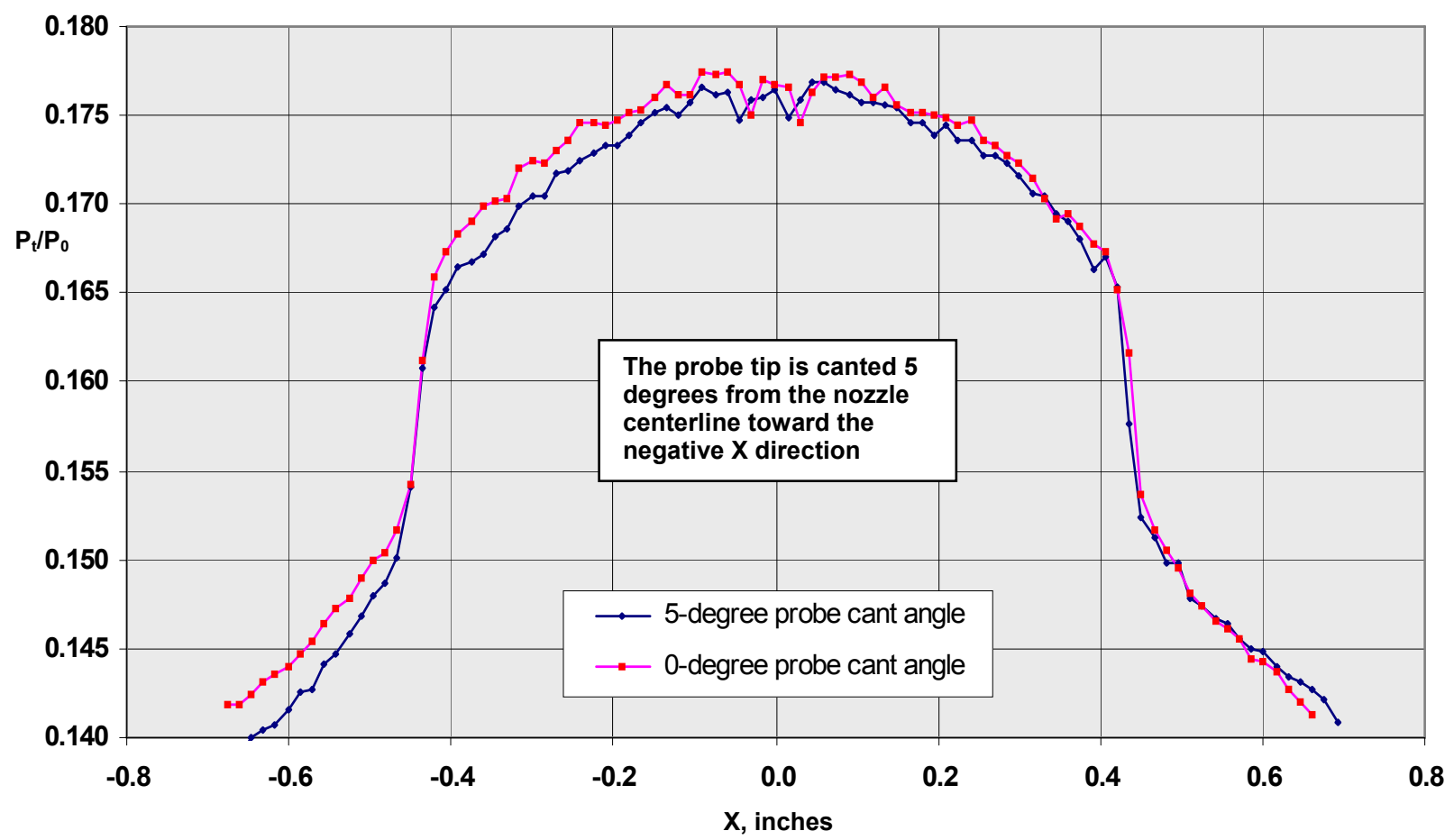

Figure 23. Comparisons of centerline Mach number surveys for probe cant angles of 0 and 5 degrees; Nozzle D-30, $P_{0}=250 \mathrm{lbs} / \mathrm{in}^{2}$. 


\section{Mach Number Survey Results}

\section{General Flow Features}

Centerline Mach number surveys derived from pitot pressure measurements can reveal a lot about the character of the flows in these scarfed nozzles. The weak compression wave noted in Figure 20 and observed in Schlieren photographs (Figure 24) moves farther from the nozzle centerline with increasing $Z$. Using the oblique conical shock charts and equations ${ }^{7}$, the strength of the wave and the change in flow direction across the wave can be estimated, given the Mach number in front of and behind the wave. From lateral survey data at $Y=0$ and $Z=0.010$ inches for Nozzle A-11-2, the Mach number ahead of the oblique shock was observed to be 3.67 and the Mach number observed behind the oblique shock was 3.50. Calculations using oblique shock relations show that the shock strength is negligible; the ratio of stagnation pressure behind the shock to stagnation pressure ahead of the shock is 0.999. Because stagnation pressure changes so little across the "oblique shock," we conclude that it is a weak (almost isentropic) compression wave. The calculations also indicate that the flow is turned by $\sim 2.6$ degrees across the wave.

Figure 24 suggests that the wave is generated downstream of the throat and crosses the nozzle centerline before it reaches the nozzle exit plane. We speculate that the wave is caused by the discontinuity in wall radius of curvature where the throat region meets the 15degree nozzle expansion section. Based upon our estimates of flow angularity upstream of the wave and our estimates of flow deflection angle across the wave, the flow downstream of the wave appears to be closely aligned with the nozzle centerline.

\section{Centerline Mach Number Correlations with Stagnation Pressure}

In order to correlate the AEDC and Ames full-scale wind tunnel vortex-fin interaction data on $J$, the value of $Q_{\text {nozzle }}$ had to be known for each supersonic spin motor nozzle configuration used in the full-scale vortex-fin interaction wind tunnel experiments. Centerline pitot pressure measurements at the exit of each nozzle were used to determine the centerline exit Mach number $M_{n o z}$ at each stagnation pressure $P_{0}$ used in the full-scale wind tunnel tests. A polynomial was used to correlate the measured values of $M_{n o z}$ with $P_{0}$ (in $1 \mathrm{bs} / \mathrm{in}^{2}$ absolute):

$$
M_{n o z}=a_{0}+a_{1} * P_{0}+a_{2} * P_{0}^{2}+a_{3} * P_{0}^{3}+a_{4} * P_{0}^{4}+a_{5} * P_{0}^{5}
$$

The polynomial fits are valid only in the interval $60 \leq P_{0} \leq 1000 \mathrm{lbs} / \mathrm{in}^{2}$, the range of stagnation pressure for which centerline Mach numbers were measured. For the nozzles with the 0.500 -inch streamwise radius of curvature (the Configuration $\mathrm{C}$ and $\mathrm{D}$ nozzles), the exit Mach number, taken from nozzle surveys at $Z=0.010$ inches in the $Y$ $=0$ plane, was a weak function of $P_{0}$. A cubic fit provided an excellent correlation for three of these nozzles. A simple linear fit was used to characterize the variation of Mach number with $P_{0}$ for nozzle C-40 because the centerline data exhibited scatter rather than a trend warranting a cubic fit. The Mach number calculated

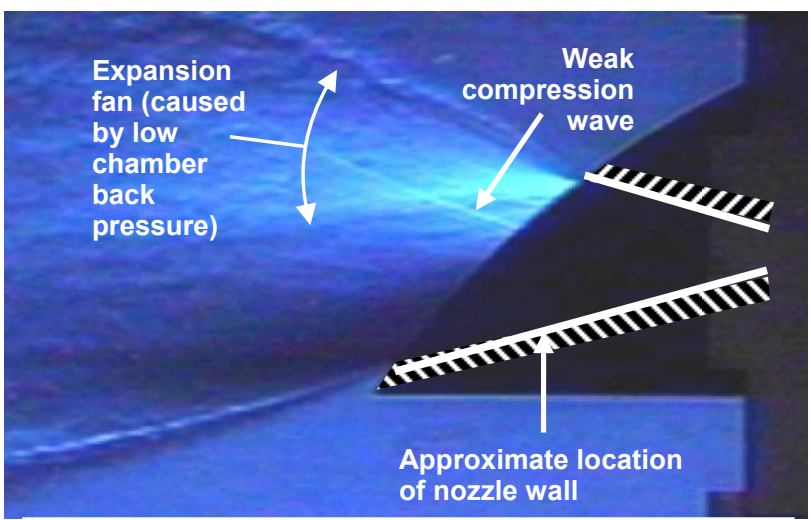

Figure 24. Schlieren photograph of the Baseline nozzle flow field. 
with the linear fit was always within 0.01 of the Mach number data obtained at the same $P_{0}$ for this nozzle. Table 3 shows the values of the coefficients for the Configuration $\mathrm{C}$ and $\mathrm{D}$ nozzles.

Table 3. Polynomial coefficients for correlating centerline nozzle exit Mach number with $P_{0}$ for Configuration $C$ and $D$ nozzles at $Z=0.010$ inches.

\begin{tabular}{|c|c|c|c|c|}
\hline$\underline{\text { Coefficient }}$ & $\underline{\text { Nozzle C-40 }}$ & $\underline{\text { Nozzle C-41 }}$ & $\underline{\text { Nozzle D-30 }}$ & $\underline{\text { Nozzle D-31 }}$ \\
\hline $\mathrm{a}_{0}$ & 3.41632 & 3.43140 & 3.68482 & 3.68667 \\
\hline$a_{1}$ & $-5.89941 \mathrm{E}-06$ & $1.43268 \mathrm{E}-04$ & 2.41972E-04 & $6.75936 \mathrm{E}-05$ \\
\hline$a_{2}$ & 0 & $-2.49374 \mathrm{E}-07$ & $-4.53235 \mathrm{E}-07$ & $-1.21017 \mathrm{E}-07$ \\
\hline$a_{3}$ & 0 & $1.07078 \mathrm{E}-10$ & $2.25629 \mathrm{E}-10$ & $3.98715 \mathrm{E}-11$ \\
\hline $\mathrm{a}_{4}$ & 0 & 0 & 0 & 0 \\
\hline$a_{5}$ & 0 & 0 & 0 & 0 \\
\hline
\end{tabular}

The nozzles with abrupt changes in wall slope near the throat (the Baseline and Configuration A and B nozzles) generated a wave system that required the use of a fifth-order polynomial to correlate $M_{n o z}$ with $P_{0}$. Correlation coefficients for each nozzle in these three configurations are given in Table 4. The centerline Mach number data for these nozzles were taken at $Z=0.0$ inches from axial surveys made along the nozzle centerline $(X=Y=0)$.

As noted earlier, the two nozzles of the same Baseline, A or B configuration had identical dimensional inspection reports but produced slightly different flow. When this was first observed, we attempted to reduce the differences by polishing one or more of the nozzles in the pair just downstream of the throat. The polished nozzles have the suffix "-2" in Table 4. Figure 25 compares the axial centerline Mach number distribution obtained for Baseline Nozzle 0 before and after it was polished. Polishing resulted in measurable changes in the centerline axial Mach number, but surveys in the $Y=0$ plane showed that polishing did not reduce the differences in the flow between the two nozzles in the pair.

Table 4. Polynomial coefficients for correlating centerline nozzle exit Mach number with $P_{0}$ for the Baseline and Configuration $A$ and $B$ nozzles at $Z=0.0$ inches.

\begin{tabular}{|c|c|c|c|c|c|c|}
\hline Coefficient & $\begin{array}{c}\text { Baseline } \\
\text { Nozzle 0-2 } \\
\end{array}$ & $\begin{array}{l}\text { Baseline } \\
\text { Nozzle } 1 \\
\end{array}$ & $\begin{array}{l}\text { Nozzle } \\
\underline{\mathbf{A}-10}\end{array}$ & $\begin{array}{l}\text { Nozzle } \\
\text { A-11-2 } \\
\end{array}$ & $\begin{array}{l}\text { Nozzle } \\
\text { B-20-2 } \\
\end{array}$ & $\begin{array}{l}\text { Nozzle } \\
\text { B-21-2 } \\
\end{array}$ \\
\hline$a_{0}$ & 3.45481 & 3.46667 & 3.36947 & 3.41153 & 3.74670 & 3.59917 \\
\hline$a_{1}$ & $\begin{array}{c}7.90953 \\
\text { E-04 }\end{array}$ & $\begin{array}{c}1.66934 \\
\text { E-04 }\end{array}$ & $\begin{array}{c}3.28652 \\
\text { E-04 }\end{array}$ & $\begin{array}{c}2.52498 \\
\text { E-04 }\end{array}$ & $\begin{array}{c}5.05802 \\
\text { E-04 }\end{array}$ & $\begin{array}{c}3.12705 \\
\text { E-04 }\end{array}$ \\
\hline$a_{2}$ & $\begin{array}{c}-3.10760 \\
\text { E-06 }\end{array}$ & $\begin{array}{c}-7.15904 \\
\text { E-07 }\end{array}$ & $\begin{array}{c}-1.17724 \\
\text { E-06 }\end{array}$ & $\begin{array}{c}-1.00061 \\
\text { E-06 }\end{array}$ & $\begin{array}{c}-1.86428 \\
\text { E-06 }\end{array}$ & $\begin{array}{c}-1.17423 \\
\text { E-06 }\end{array}$ \\
\hline$a_{3}$ & $\begin{array}{c}5.68660 \\
\text { E-09 }\end{array}$ & $\begin{array}{c}1.29195 \\
\text { E-09 }\end{array}$ & $\begin{array}{c}1.66209 \\
\text { E-09 }\end{array}$ & $\begin{array}{c}1.63305 \\
\text { E-09 }\end{array}$ & $\begin{array}{c}3.46230 \\
\text { E-09 }\end{array}$ & $\begin{array}{c}2.04909 \\
\text { E-09 }\end{array}$ \\
\hline$a_{4}$ & $\begin{array}{c}-4.93375 \\
\text { E-12 }\end{array}$ & $\begin{array}{c}-1.18591 \\
\text { E-12 }\end{array}$ & $\begin{array}{c}-9.42774 \\
\text { E-13 }\end{array}$ & $\begin{array}{c}-1.31340 \\
\mathrm{E}-12\end{array}$ & $\begin{array}{c}-3.09584 \\
\text { E-12 }\end{array}$ & $\begin{array}{c}-1.86317 \\
\text { E-12 }\end{array}$ \\
\hline$a_{5}$ & $\begin{array}{c}1.62395 \\
\text { E-15 }\end{array}$ & $\begin{array}{c}4.26694 \\
\text { E-16 }\end{array}$ & $\begin{array}{c}1.78733 \\
\text { E-16 }\end{array}$ & $\begin{array}{c}4.11246 \\
\text { E-16 }\end{array}$ & $\begin{array}{c}1.05432 \\
\text { E-15 }\end{array}$ & $\begin{array}{c}7.26084 \\
\text { E-16 }\end{array}$ \\
\hline
\end{tabular}




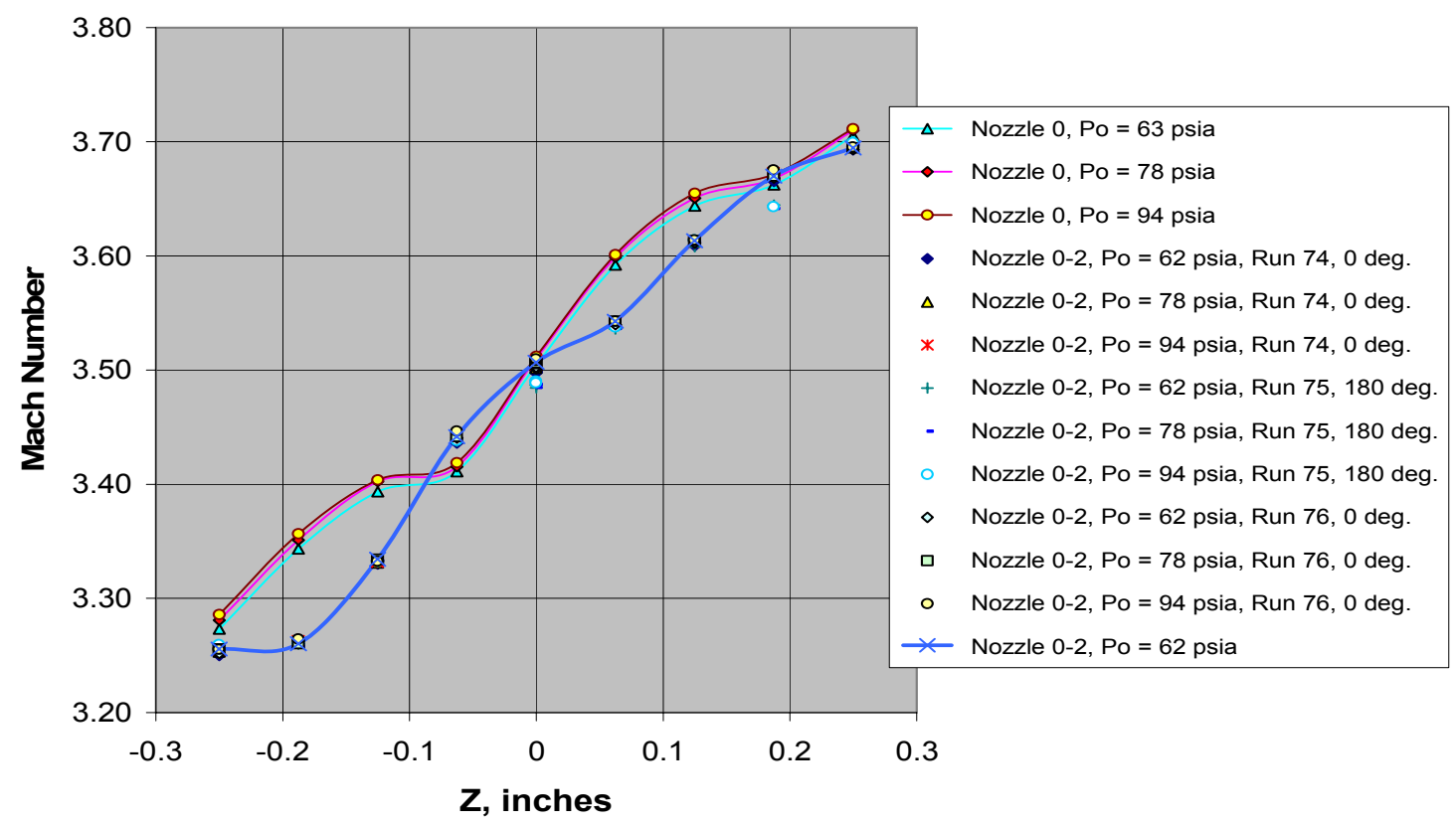

Figure 25. Comparison of centerline axial Mach number $(X=Y=0)$ for Baseline Nozzle 0 (before polishing) and Baseline Nozzle 0-2 (after polishing).

\section{Baseline Nozzle Survey Results}

The Mach number surveys on the $Y=0$ plane for $Z=0.015$ inches are shown for Baseline Nozzles $0-2$ and 1 in Figure 26. The surveys were made at a stagnation pressure of 100 $1 \mathrm{bs} / \mathrm{in}^{2}$. They show reasonable flow uniformity $( \pm 0.03)$ across the nozzle at a fixed $Z$ survey

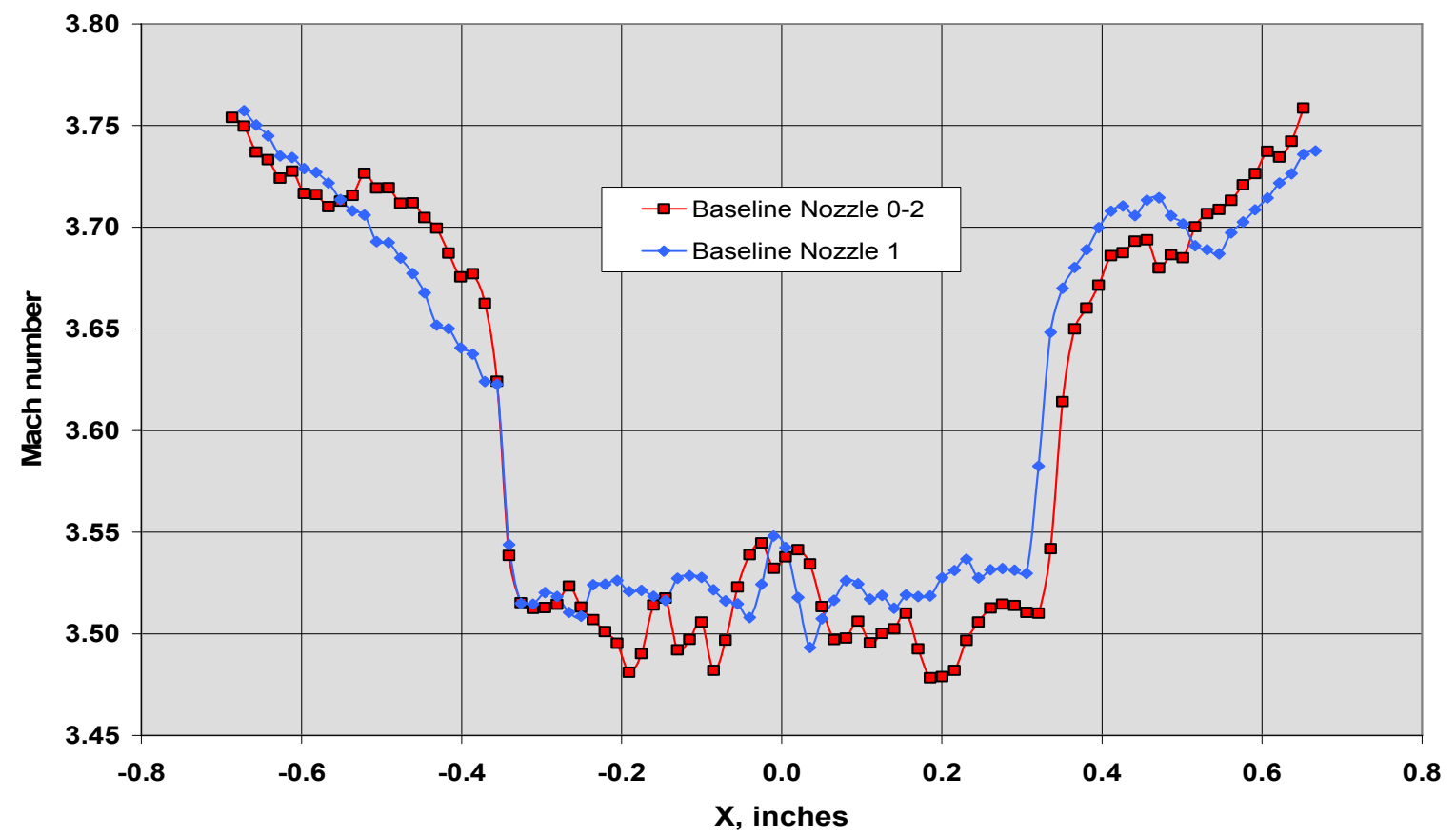

Figure 26. Comparison of Baseline Nozzle 0-2 and Nozzle 1 Mach number surveys on the $Y=0$ plane for $Z=0.015$ inches and $P_{0}=100 \mathrm{lbs} / \mathrm{in}^{2}$. 
station. This was surprising, given the fact that no attempt was made to achieve good flow uniformity by contouring the nozzle walls or by using a nozzle configuration with continuous streamwise radius of wall curvature through the throat. But the flow fields are different in subtle ways: although the basic wave structures are the same in each profile, the detailed structure is not. Furthermore, both nozzle surveys are slightly asymmetric. A detailed inspection of the nozzles in the machine shop showed no measurable differences in throat geometry and dimensions. However, we believe that such differences do exist, and that they are the cause of measurable differences in the flow field even though the differences in throat geometry and dimensions are not measurable. This finding means that the flow fields produced by these nozzles cannot be used for computational fluid dynamics code validation.

Figures 27 and 28 show the lateral Mach number survey profiles for Baseline nozzles 0 and 1 , respectively, for $62<P_{0}<1000$. Reynolds number increases in proportion to $P_{0}$, and the thickness of the nozzle wall boundary layer decreases with increasing Reynolds number. The survey results from both nozzles show that the basic wave structure that characterizes the inviscid flow remains the same, independent of Reynolds number. Varying stagnation pressure from 63 to $1000 \mathrm{lbs} / \mathrm{in}^{2}$ causes only small changes in the inviscid flow. The $X$-location of the weak compression wave at this axial survey station moved closer to the centerline $(X=0)$ as stagnation pressure was increased. The Mach number profiles changed by only $\sim 0.03$ over this range of stagnation pressure. Upstream of the weak compression wave $(X<-0.3$ and $X>+0.3)$, Mach number at any survey location generally increases with $P_{0}$. Downstream of the weak compression wave $(-0.3 \geq X \geq+0.3)$, the changes in Mach number with $P_{0}$ were not monotonic; the Mach numbers in this region of the flow are slightly higher for $P_{0}=375 \mathrm{lbs} / \mathrm{in}^{2}$ than for either $P_{0}=63 \mathrm{lbs} / \mathrm{in}^{2}$ or $900 \mathrm{lbs} / \mathrm{in}^{2}$.

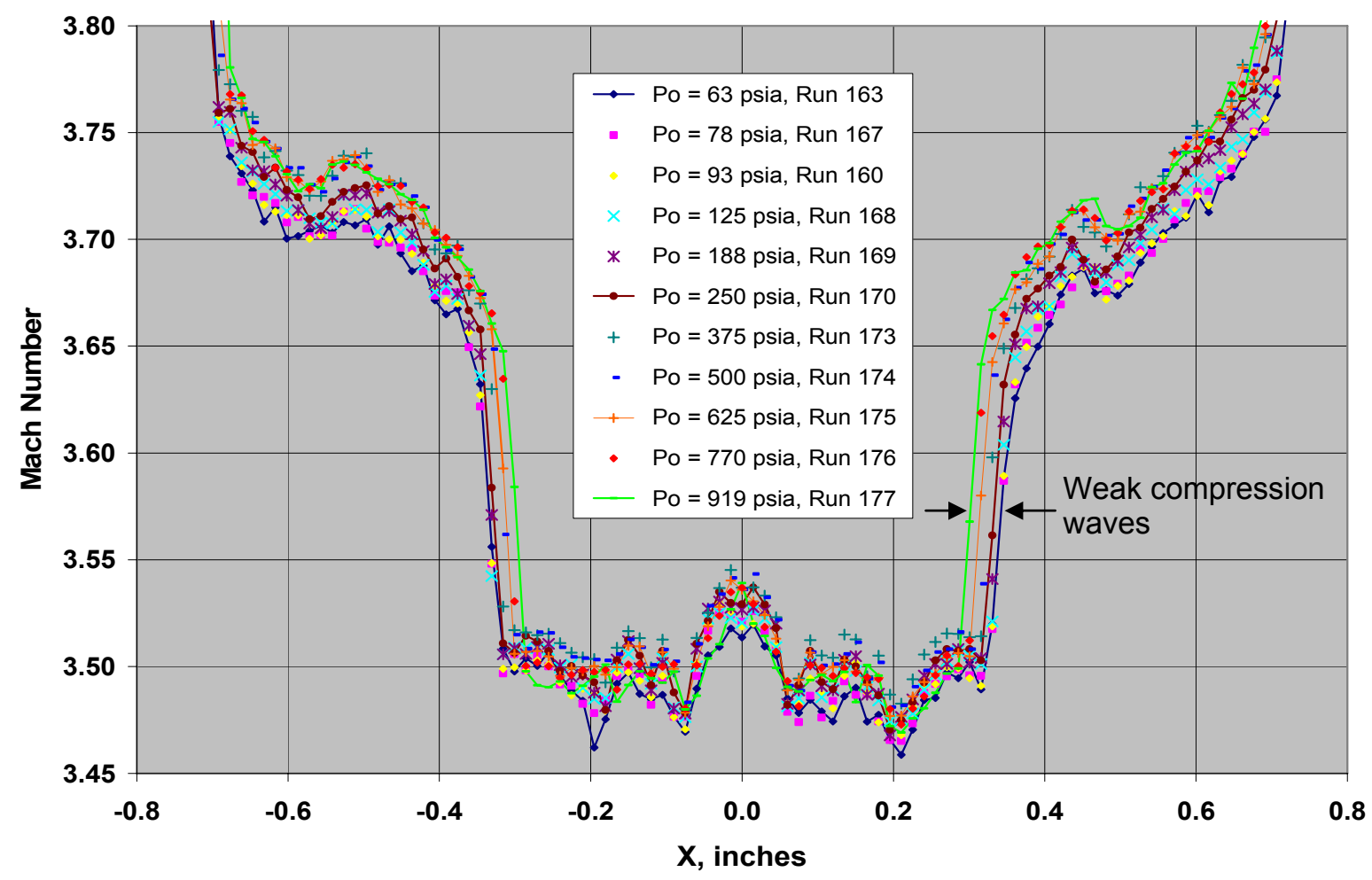

Figure 27. Mach number surveys in the $Y=0$ plane at $Z=0.010$ inches for Baseline Nozzle 0-2. 


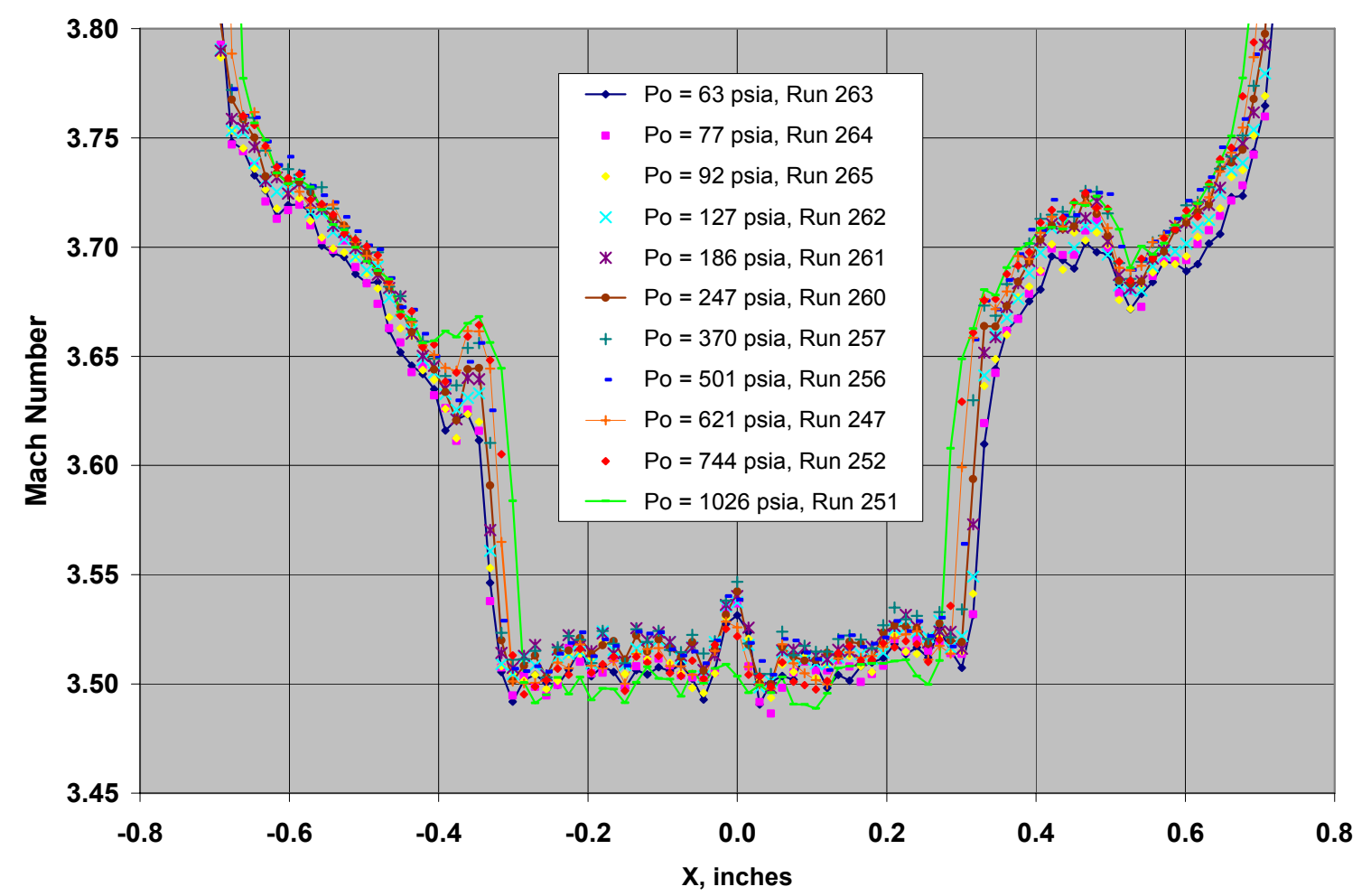

Figure 28. Mach number surveys in the $Y=0$ plane at $Z=0.010$ inches for Baseline Nozzle 1.

These observed flow field variations with $P_{0}$ suggest that these nozzles produce an inviscid flow that responds to (small) changes in boundary layer thickness as Reynolds number is varied. Changes in the boundary layer displacement thickness change the location and magnitude of the inviscid flow features, but not their basic character.

Surveys at the exit plane of Baseline Nozzles 0-2 and 1 are presented in Figures 29 and 30, respectively. Recall that the nozzle exit plane is defined as the intersection of the nozzle with the cylindrical surface (outer skin) of the B61. The dimension $Z^{\prime}$ is defined as the distance downstream of the nozzle exit plane as measured in the $X=0$ plane. Surveys were intentionally made at small positive values of $Z^{\prime}$ in order to avoid running the probe tip into the nozzle block at the nozzle wall. Each family of surveys were taken so that an average value of the vortex-fin interaction correlation parameter $J$ could be found by converting the Mach numbers to dynamic pressure at each survey point, and then determining the average value of dynamic pressure across the nozzle exit plane.

For Baseline Nozzle 0-2 (Figure 29), the probe is completely outside (upstream) of the weak compression wave for $Y$ survey stations less than -0.250 inches and greater than +0.250 inches. At these survey stations, the Mach number is very uniform across the entire inviscid nozzle flow field. As one would expect, the Mach number is lower on the "short" side of the nozzle and higher on the "long" side of the nozzle. At survey stations where $-0.250 \leq Y \leq$ +0.250 , these trends of increased Mach number with increasing $Y$ and the uniform flow are observed outside of the weak compression wave. Flow non-uniformity is observable inside (downstream of) the weak compression wave, particularly for the survey at $Y=-0.250$ inches. For Baseline Nozzle 1 (Figure 30), the same trends are observed, but the flow inside the weak compression wave is much more uniform than it is for Baseline Nozzle 0-2. 


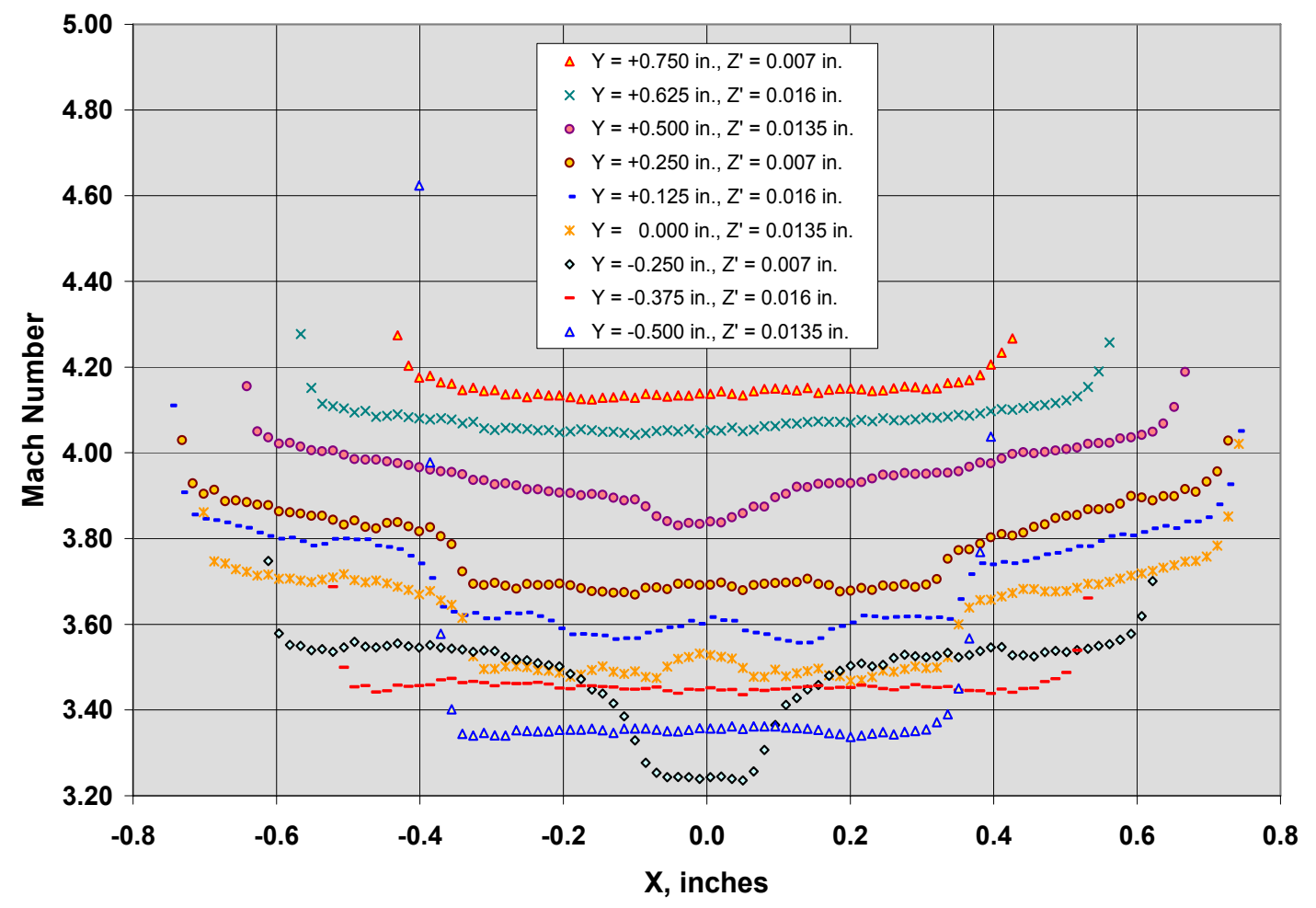

Figure 29. Nozzle exit plane Mach number surveys for Baseline Nozzle 0-2.

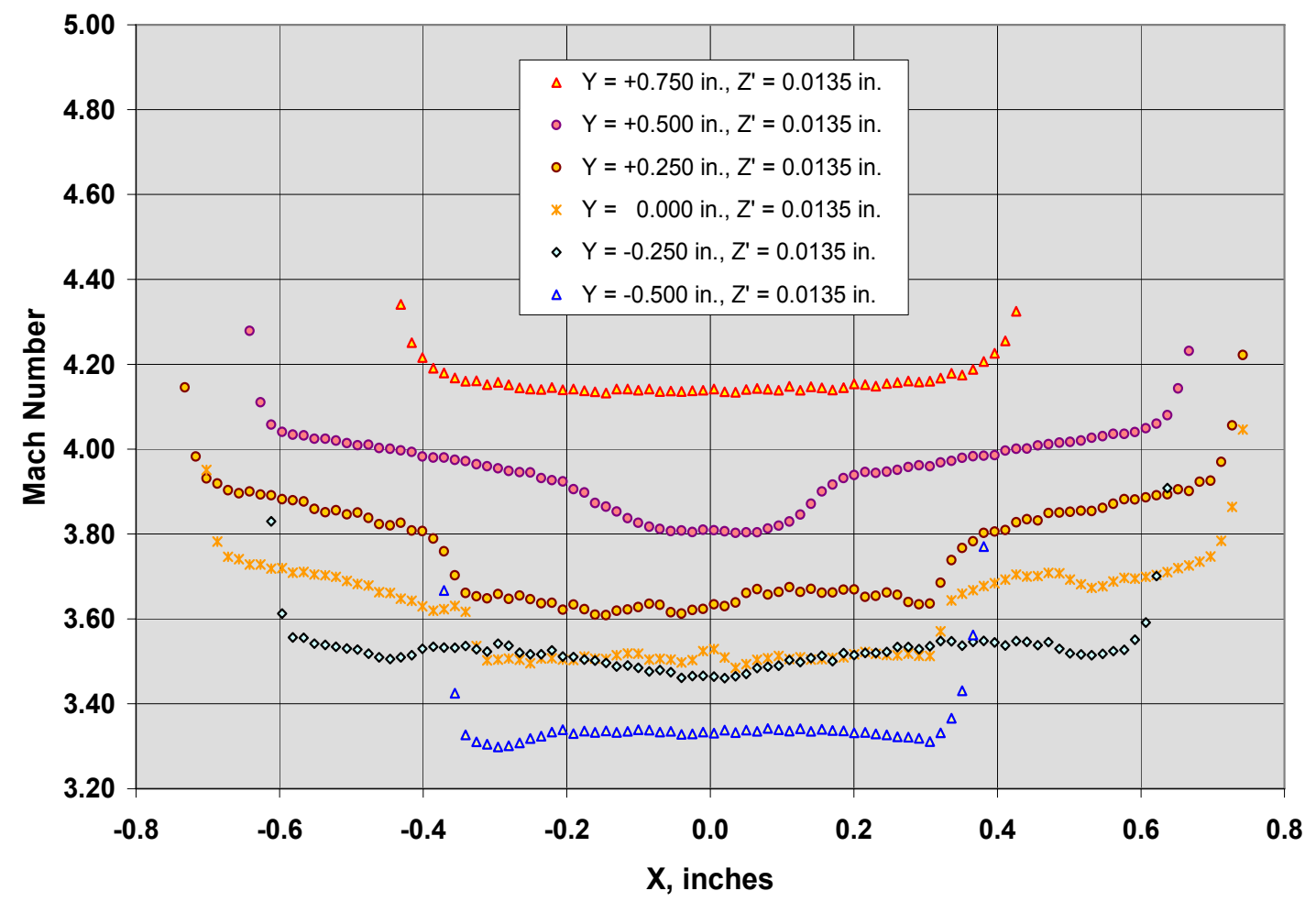

Figure 30. Nozzle exit plane Mach number surveys for Baseline Nozzle 1. 


\section{Nozzle Configuration A and B Survey Results}

Mach number survey results in the $Y=0$ plane at $Z=0.010$ inches are shown for the Configuration A-10 and A-11-2 nozzles in Figures 31 and 32, and for the Configuration B-20-2 and B-21-2 nozzles in Figures 33 and 34. Recall that all Configuration A and B nozzles have a smaller throat diameter than the Baseline nozzles $(0.476$ inches instead of 0.535 inches). Therefore, we might expect that the same differences in the Baseline nozzle throat geometry could have an even larger effect upon the flow in the Configuration A and B nozzles than they had in the Baseline nozzles. The data qualitatively confirm this expectation. Specific observations:

- The differences in the flow generated by each nozzle in a Configuration A and B nozzle pair are larger than the differences in the flows produced by Baseline nozzles $0-2$ and 1.

- The flow in each Configuration $\mathrm{A}$ and $\mathrm{B}$ nozzle is quite asymmetric at all $P_{0}$ values.

- Some of the asymmetric flow features change with Reynolds number (stagnation pressure). This is especially true of Nozzle Configuration A-11-2, where a sudden dip in Mach number is observed at $X=-0.2$ inches at $P_{0}=64 \mathrm{lbs} / \mathrm{in}^{2}$. As stagnation pressure is increased, the dip in Mach number at $X=-0.2$ inches disappears, and an increase in Mach number appears at $X=+0.25$ inches. It seems as if whatever is causing the dips/increases moves around the nozzle as the nozzle Reynolds number increases.

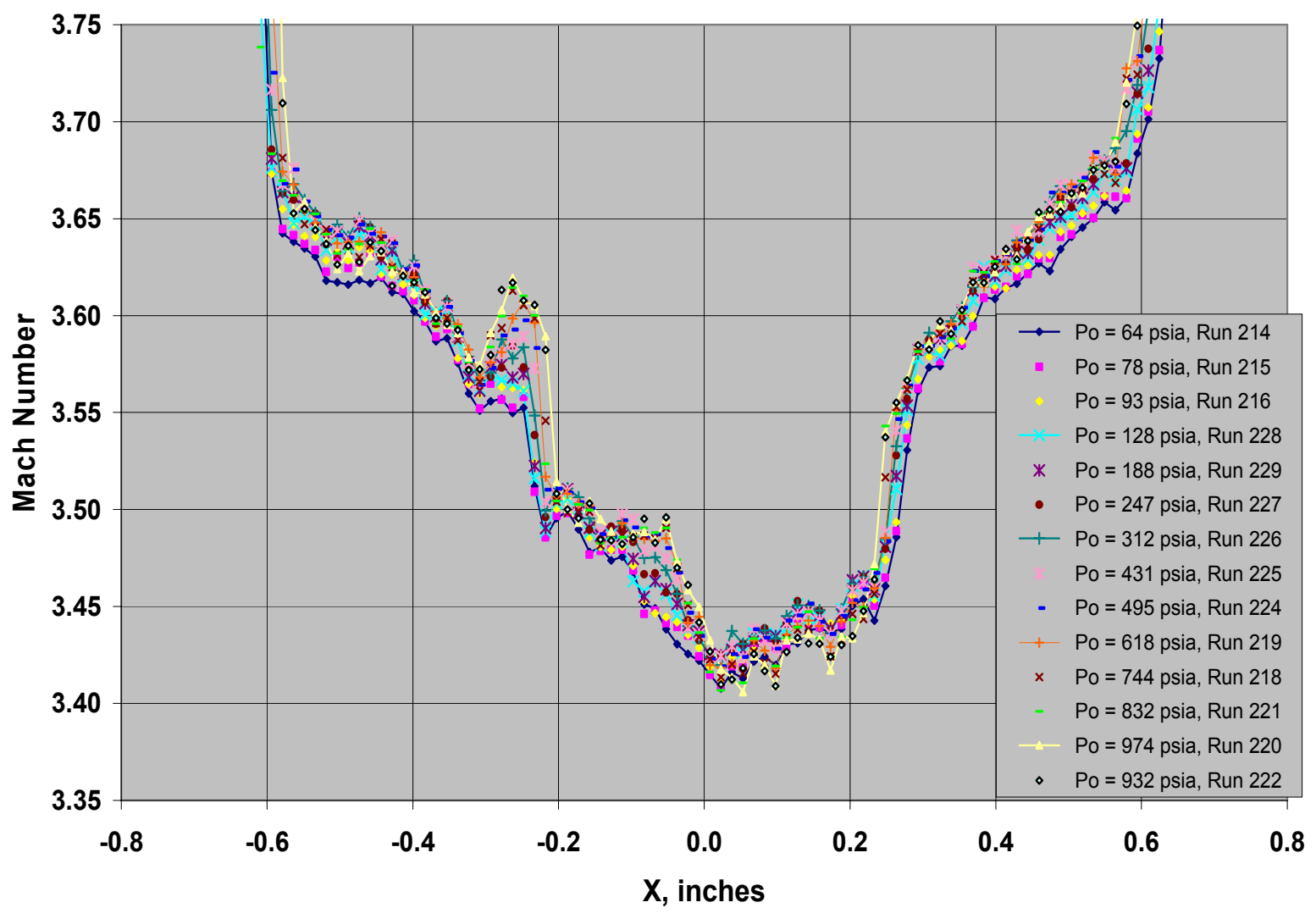

Figure 31. Mach number surveys in the $Y=0$ plane at $Z=0.010$ inches for Nozzle Configuration A-10. 


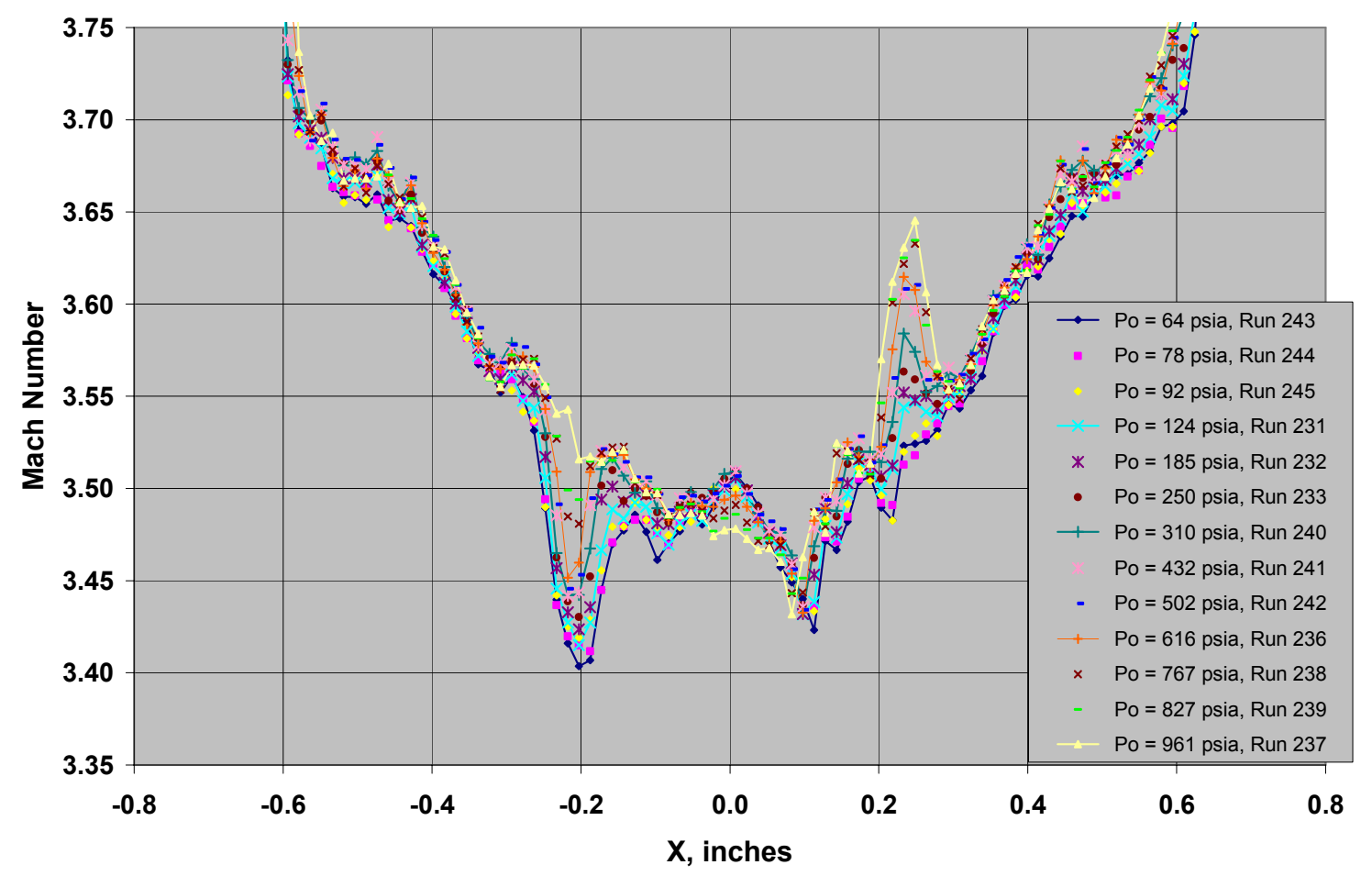

Figure 32. Mach number surveys in the $Y=0$ plane at $Z=0.010$ inches for Nozzle Configuration A-11-2.

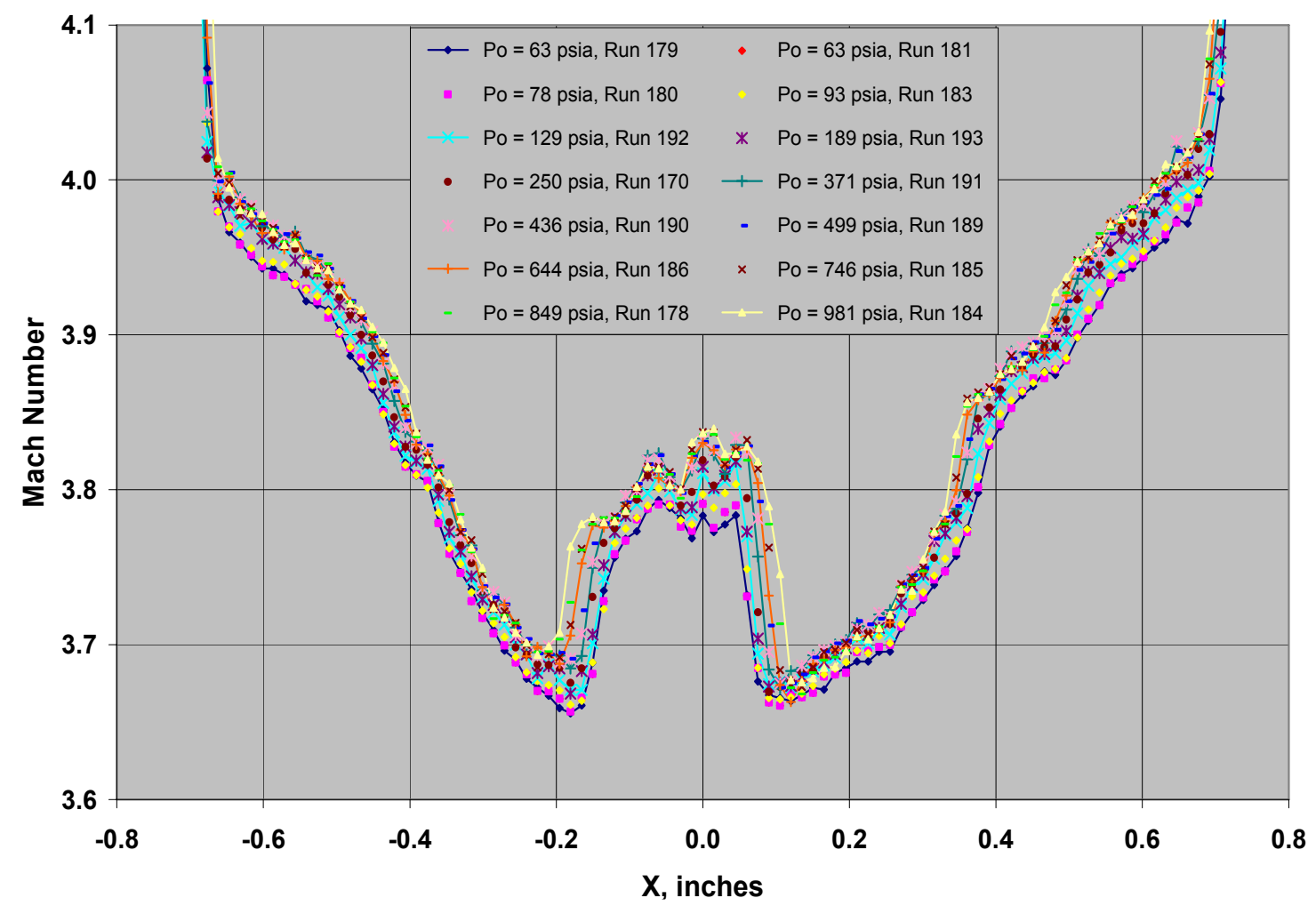

Figure 33. Mach number surveys in the $Y=0$ plane at $Z=0.010$ inches for Nozzle Configuration B-20-2. 


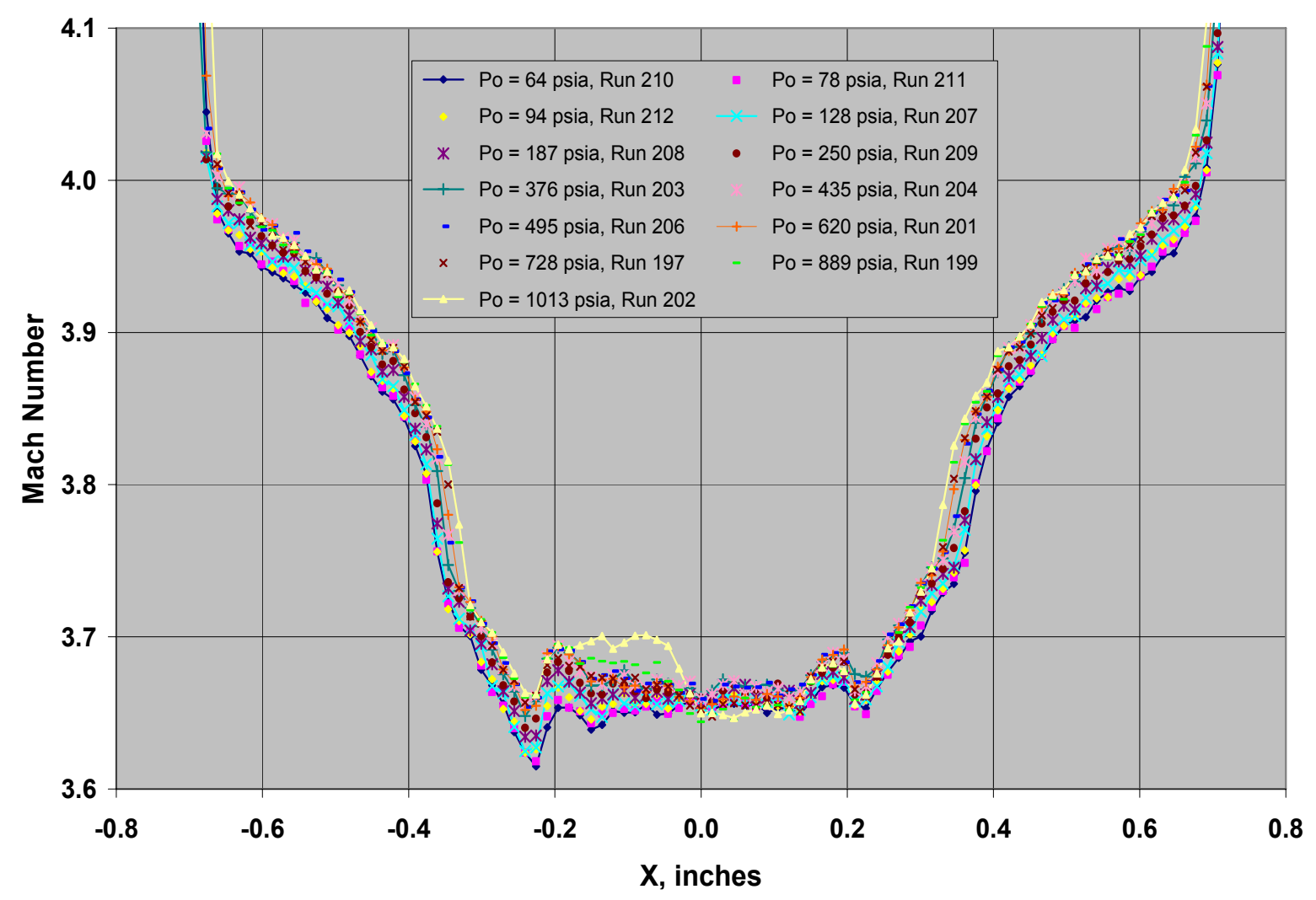

Figure 34. Mach number surveys in the $Y=0$ plane at $Z=0.010$ inches for Nozzle Configuration B-21-2.

- Recall that centerline values of Mach number are used to calculate $J$, the parameter used to correlate the reduction in roll torque due to vortex-fin interaction. Because of the asymmetries and variation of Mach number observed in the Configuration A and $B$ nozzles, the centerline value of Mach number may not be representative of the full inviscid flow.

Because of the poor flow quality produced by unknown and/or unmeasurable geometric features, data from Configuration A and B nozzles should not be used to validate CFD codes.

\section{Nozzle Configuration C and D Survey Results}

Centerline exit Mach number surveys ( $Y=0, Z=0.010$ in.) for Configuration C-40 and C-41 nozzles are shown in Figures 35 and 36. The inviscid flow features and Mach numbers change very little (by only a few hundredths of a Mach number) at any given flow field location as the nozzle stagnation pressure varies from 62 to $1000 \mathrm{lbs} / \mathrm{in}^{2}$. The same is true for the centerline exit Mach number survey results from Nozzle Configurations D-30 and D-31 (Figures 37 and 38). As noted earlier, the two nozzles in each pair produce nearly identical flow fields. We believe that the data from these nozzles is unique and traceable to these specific nozzle geometries, and therefore the data can be used for CFD code validation. 


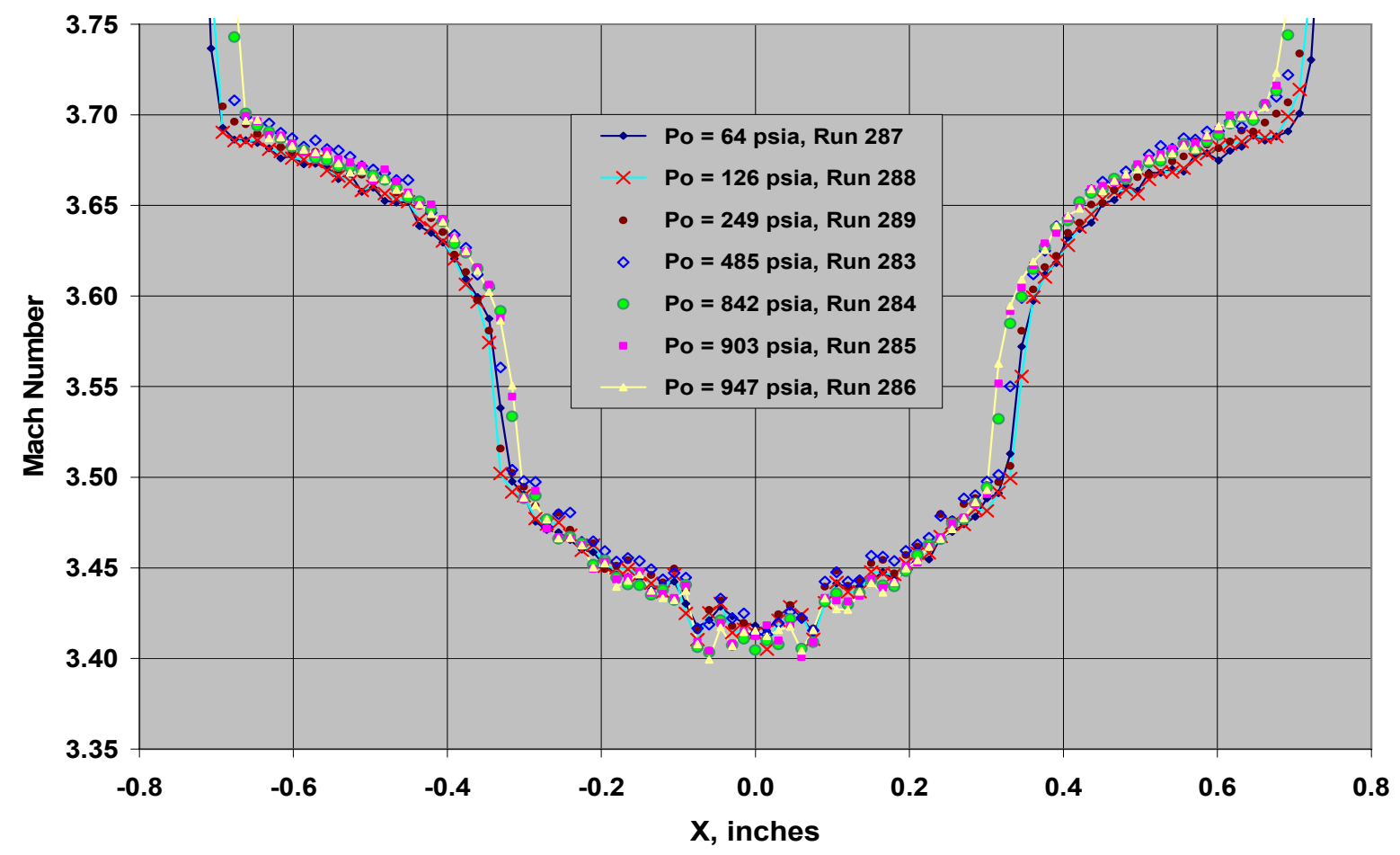

Figure 35. Nozzle Configuration C-40 centerline exit Mach number survey results.

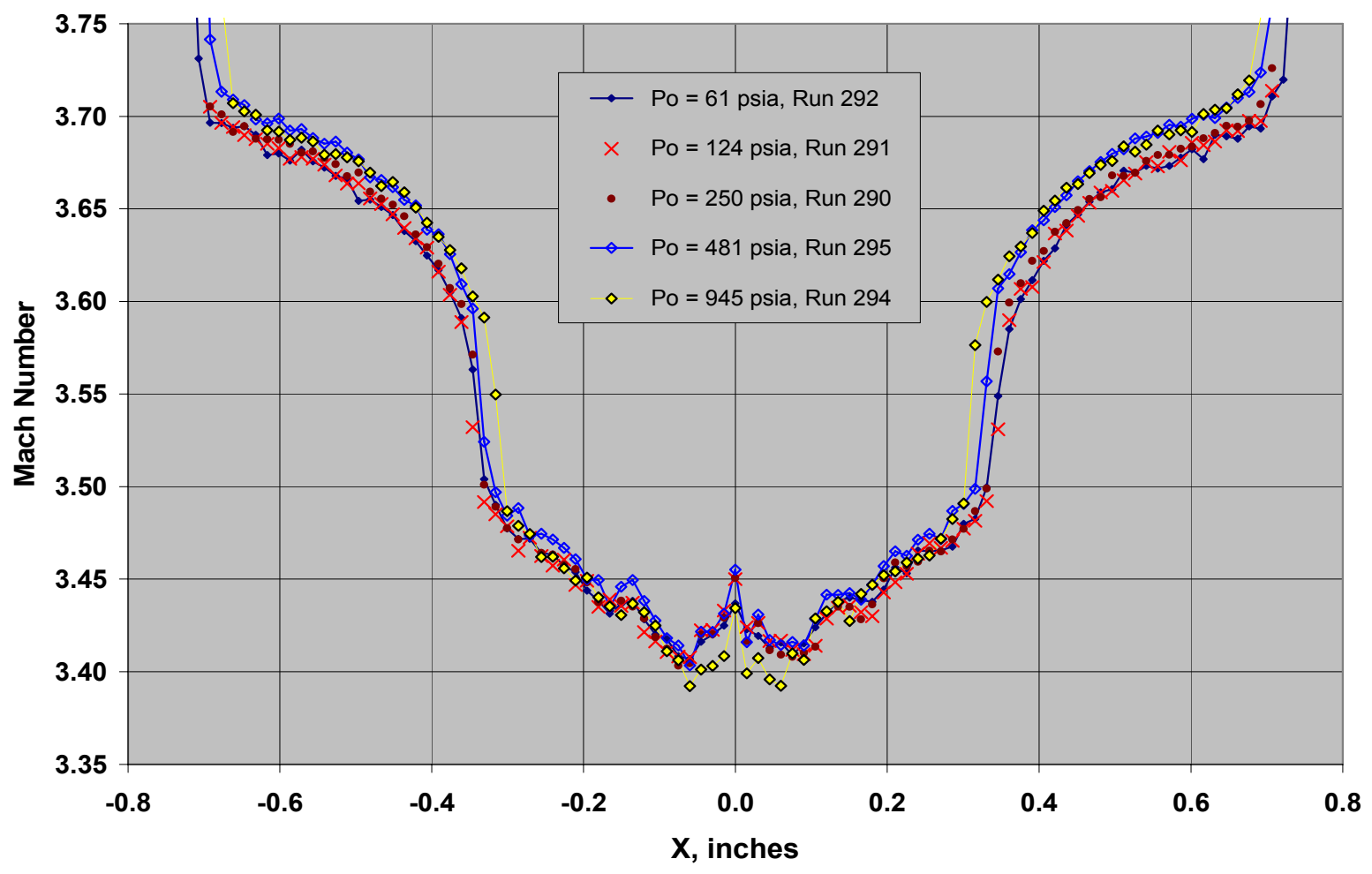

Figure 36. Nozzle Configuration C-41 centerline exit Mach number survey results. 


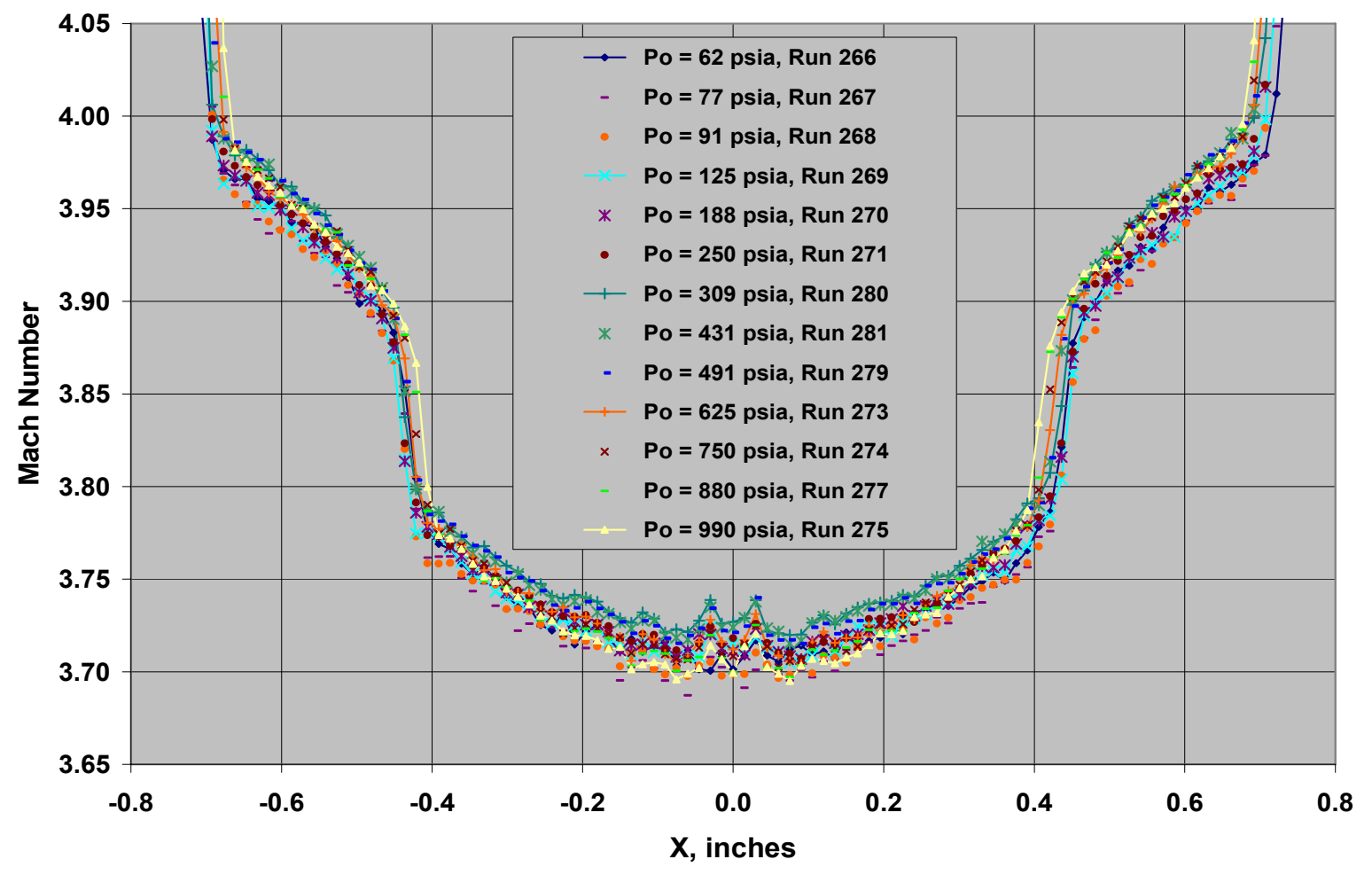

Figure 37. Nozzle Configuration D-30 centerline exit Mach number survey results.

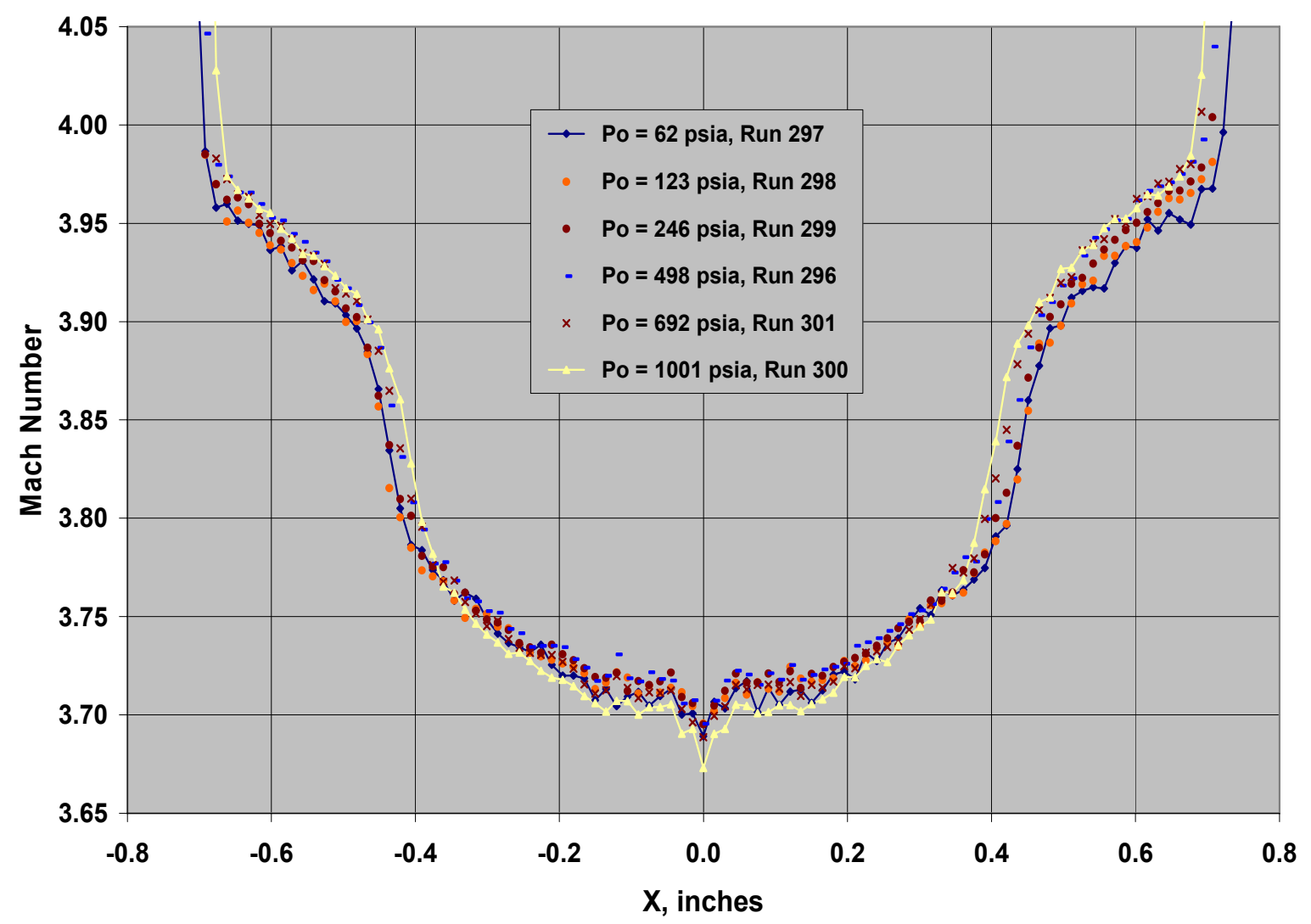

Figure 38. Nozzle Configuration D-31 centerline exit Mach number survey results. 
Figures 39a-c compare the Nozzle C-40 and C-41 exit flow Mach number surveys $(Y=0, Z=$ 0.010 in.) to the exit surveys for Baseline Nozzles 0-2 and 1. Recall that the ratio of throat area to exit area is the same for each of these four nozzles; the only intentional difference is associated with the geometry of the throat region. To the best of our ability to measure, the Baseline nozzles have the same throat geometry as is used in flight, whereas the two Configuration $\mathrm{C}$ nozzles have a 0.500 -inch streamwise radius of curvature throughout the throat region. The survey data show that changing to the 0.500 -inch streamwise radius of curvature in the throat region makes a large difference in the $Y=0$ plane nozzle exit flow field. More specifically,

- Over the full range of nozzle stagnation pressures, the two Configuration $\mathrm{C}$ nozzles produce nearly identical flows, whereas the two Baseline nozzles do not. We conclude that the 0.500 -inch streamwise radius of curvature in the throat region of the Configuration $\mathrm{C}$ nozzles eliminated the problem that produced both flow asymmetries and measurable differences between the flow fields of the two Baseline nozzles.

- Even though the geometric ratio of throat area to exit area is the same for each of these four nozzles, the Mach number throughout the inviscid exit flow of the Baseline nozzles is somewhat higher than the Mach number observed in the Configuration $\mathrm{C}$ nozzles. This result implies that the effective throat diameter of the Baseline nozzles is smaller than the effective throat diameter of the Configuration $\mathrm{C}$ nozzles. A reduction in the effective diameter of the Baseline nozzles could be caused by separation of the flow as it approaches the throat at $90^{\circ}$ to the nozzle axis and then attempts to align itself with the nozzle axis. Recall that the radius of curvature immediately upstream of the throat was less than 0.020 inches. It seems quite likely that the flow cannot follow the wall contour around such a small radius of curvature and therefore separates in the throat.

- Notice that the weak compression wave is observed in all Baseline and Configuration C nozzles, and that its strength (determined by the Mach number jump across it) is independent of the nozzle throat configuration. From this observation, we conclude that the weak compression wave is not formed as a by-product of separation in the nozzle throat (there is no evidence of separation in the Configuration $\mathrm{C}$ nozzles, but there is in the Baseline nozzles). As stated earlier, we speculate that the cause of the weak compression wave is the discontinuity in the wall radius of curvature where the nozzle throat transitions to the $15^{\circ}$ conical expansion section. The angular momentum of the flow near the wall must suddenly go to zero at this discontinuity; the process of doing so may give rise to the weak compression wave.

Figures 40a-c compare the centerline exit flow surveys from the Configuration D nozzles (which have the 0.500-inch streamwise radius of curvature in the throat region) with Configuration B nozzle surveys at three different nozzle stagnation pressures. The Configuration D nozzles produce nearly identical symmetric flow fields, in sharp contrast to the Configuration B nozzles, whose flows differ greatly and are not symmetric. Also note that the weak compression wave in the Configuration D nozzle surveys has moved approximately 0.05 inches further from the nozzle centerline than its counterpart in the Configuration B nozzles. 

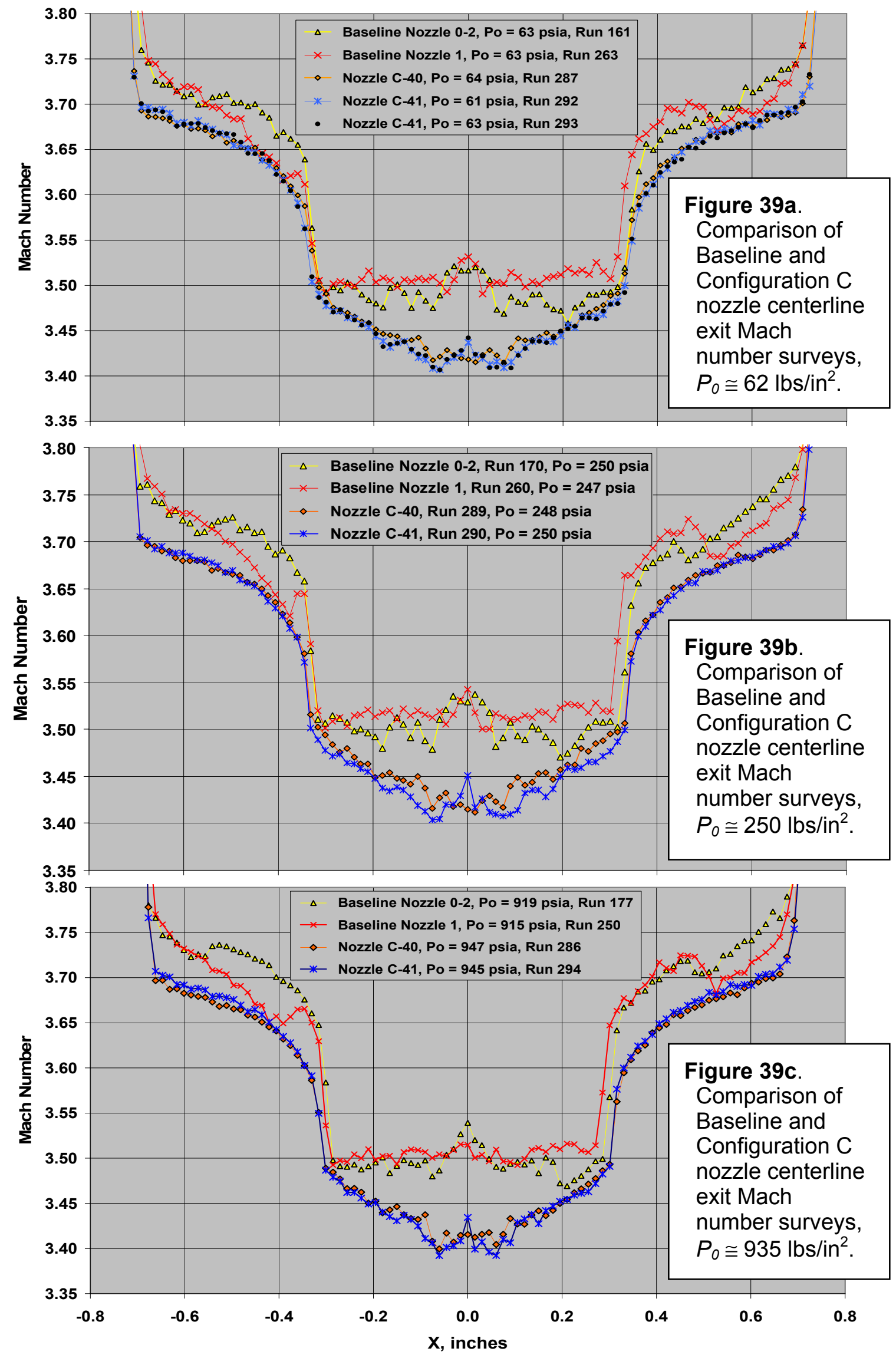

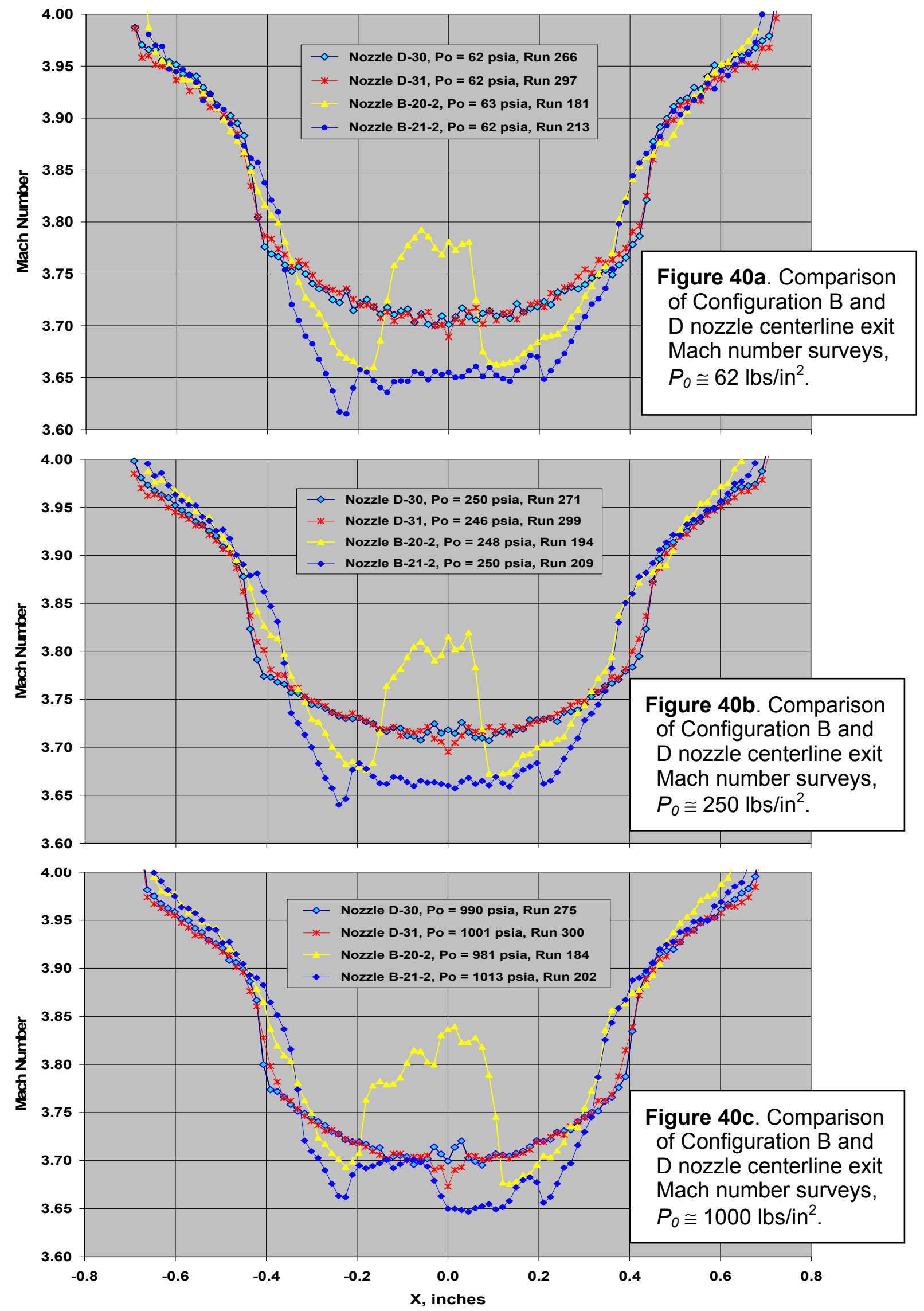
The "+" symbols in Figure 41 show the locations of surveys made across the exit plane (the intersection of the nozzle contour with the cylindrical outer surface of the flight vehicle) for both Configuration $\mathrm{C}$ and $\mathrm{D}$ nozzles. The purpose of these surveys was to characterize the flow field across the entire nozzle exit plane (not just at the nozzle exit on the nozzle centerline) in order to estimate the average exit nozzle dynamic pressure over the nozzle exit plane. For axisymmetric nozzles, the average dynamic pressure is closely approximated by the exit plane value on the nozzle centerline $(Y=0)$. For scarfed nozzles like those used in this experiment, the average dynamic

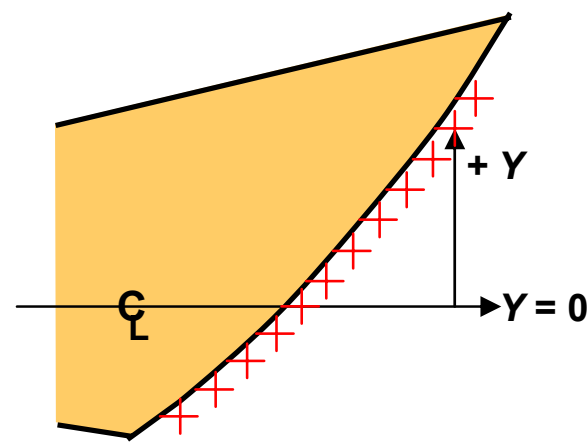

Figure 41. Location of pitot probes for surveys across the nozzle exit plane. pressure could be quite different from the exit centerline value because of the range of Mach numbers between the "short" and "long" sides of the nozzle. Figure 41 shows that positive $Y$ is in the direction of the "long side" of the scarfed nozzle and negative $Y$ is in the direction of the "short side." Pitot measurements were made at $Z^{\prime}$ locations of +0.0100 or +0.015 inches downstream of the actual nozzle exit plane to prevent the probe tips from contacting the nozzle walls at either end of the surveys.

Figure 42 shows the nozzle exit plane Mach number profiles at $Y=+0.875,+0.750,+0.635$, $+0.500,+0.375,+0.250,+0.125,0.000$ (centerline), $-0.125,-0.250,-0.375$ and -0.500 inches for Nozzle Configuration C-40 at stagnation pressure of $150 \mathrm{lbs} / \mathrm{in}^{2}$. Although complete Nozzle C-40 exit plane surveys were also obtained for at $P_{0}=100,400$ and $500 \mathrm{lbs} / \mathrm{in}^{2}$, only the results at $150 \mathrm{lbs} / \mathrm{in}^{2}$ are shown because the data show a very weak dependence on stagnation pressure. The surveys at $Y=-0.500$ and -0.375 inches are both upstream of the weak compression wave. The Mach number is very uniform across the inviscid flow field at these survey locations. Because the $Y=-0.500$ survey is closest to the nozzle throat, its Mach number is lowest. The pitot probe is downstream of the weak compression wave at $Y=$ -0.250 inches, and as a result, the Mach number downstream of the weak compression wave at this survey location is lower than the Mach number upstream of the weak compression wave at $Y=-0.370$ inches. The probe remains downstream of the weak compression wave over at least part of the surveys from $Y=-0.250$ inches to $Y=+0.525$ inches. The Mach numbers increase with increasing $Y$ because the survey locations are progressively further from the nozzle throat. The probe is again upstream of the weak compression wave for the $Y$ $=+0.750$ - and +0.875 -inch surveys. Because there is no constant Mach number region in the $Y=+0.875$-inch survey (as there is in the survey at $Y=+0.750$ inches), it is possible that the probe was in the nozzle wall boundary layer, in which case these data should be ignored.

Similar trends are observed in the nozzle exit plane Mach number survey data taken for Nozzle D-30 at a stagnation pressure of $500 \mathrm{lbs} / \mathrm{in}^{2}$ (Figure 43). The only differences between C-40 and D-30 survey results are the higher Mach numbers measured in the D-30 nozzle due to its higher expansion ratio. As was also the case for Nozzle C-40, the Mach number is very uniform across the inviscid flow field produced by Nozzle D-30 at these survey locations. 


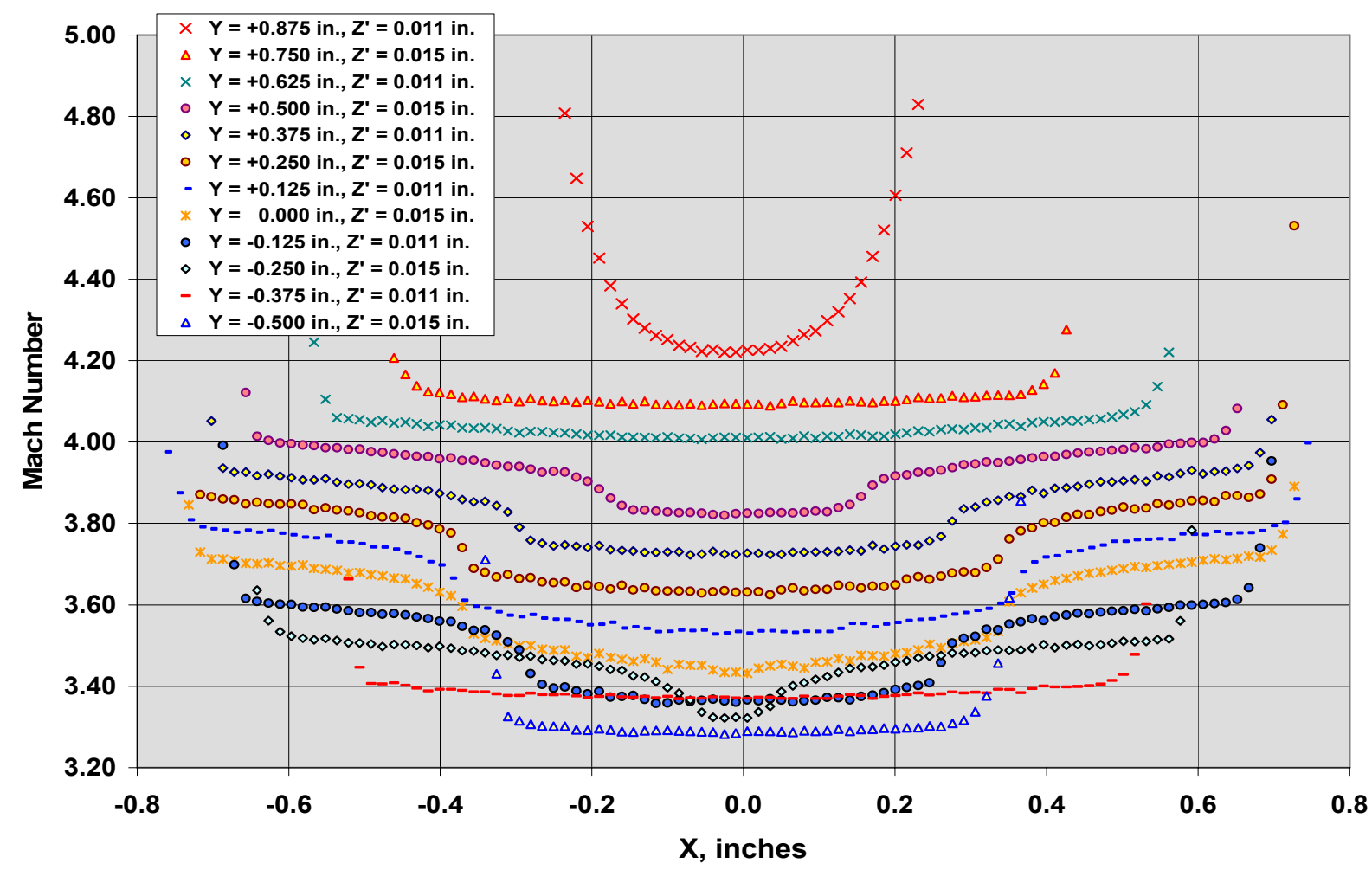

Figure 42. Mach number profiles obtained at twelve $Y$ locations across the Configuration C-40 nozzle exit plane, $P_{0}=150 \mathrm{lbs} / \mathrm{in}^{2}$.

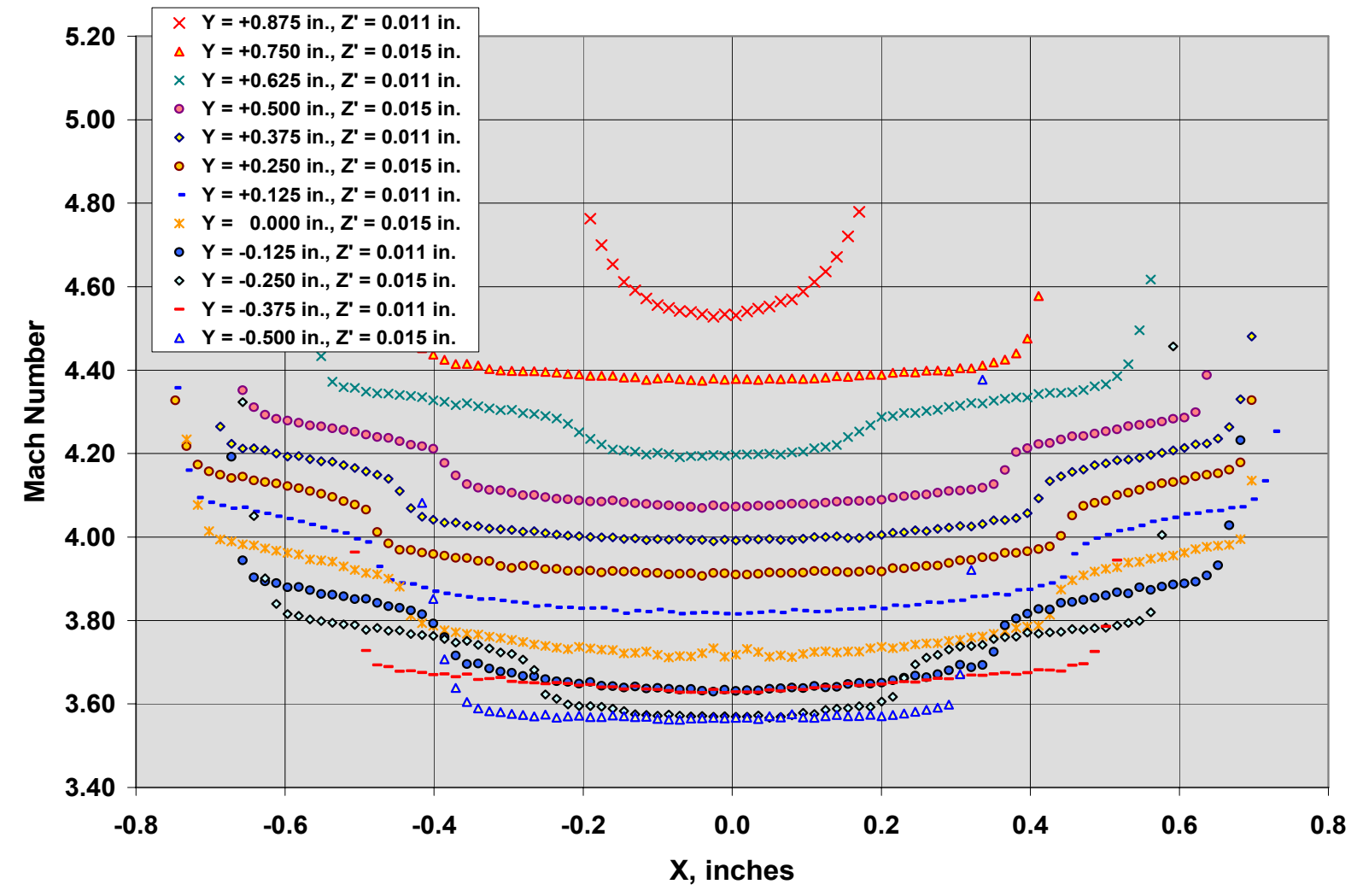

Figure 43. Mach number profiles obtained at twelve $Y$ locations across the Configuration D-30 nozzle exit plane, $P_{0}=500 \mathrm{lbs} / \mathrm{in}^{2}$. 


\section{Summary and Conclusions}

Flight test data showed that the spin rate produced by spin motors on finned vehicles can be lower than expected as a result of "vortex-fin interaction," a complex flow field interaction with the vehicle's fins. Neither experimental data nor validated Computational Fluid Dynamics predictions could be found that characterized the primary fluid dynamic and vehicle configuration parameters affecting the magnitude of the loss of spin rate caused by vortex-fin interaction. To fill this void, full-scale wind tunnel tests were conducted to measure the counter torque caused by vortex-fin interaction. The flow coming out of the spin motor nozzles had to be characterized in the absence of the transonic crossflow over the flight vehicle in order to determine the initial boundary conditions to the vortex-fin interaction phenomenon. In these experiments, the Mach number across the exit plane in the inviscid core of scarfed supersonic nozzles was determined using pitot probes.

Actual flight vehicle spin motors were not used in the full-scale wind tunnel experiments because only one data point could be obtained for each motor firing and because of the extra time that would have been needed to replace expended motors between runs. Surrogate nozzles were designed to use high-pressure air instead of the combustion products produced by the flight vehicle's spin motors. The flight vehicle spin nozzles were replaced by Baseline wind tunnel model nozzles that had the same exit dimensions as the flight vehicle spin nozzles, but the throat diameter was increased in order to generate the same exit centerline Mach number using air as was calculated for the flight vehicle nozzles. The Baseline nozzles used the same throat geometry as the flight vehicle nozzle configuration. This throat geometry featured an abrupt, $90^{\circ}$ transition of the wall slope to the nozzle throat followed by a constant throat diameter over a length of $\sim 0.15$ inches. Two other nozzles with the same throat geometry but with a smaller throat diameter were also studied. A fourth and fifth nozzle configuration, each having a 0.500 -inch-diameter streamwise radius of curvature through the throat region, were proposed as a means of improving flow quality and symmetry relative to that produced by the nozzles that mimicked the flight vehicle nozzle's throat geometry.

Nozzle characterization experiments were conducted in a modified section of an obsolete $\mathrm{M}$ = 7.3 nozzle assembly on Sandia's Hypersonic Wind Tunnel. By capitalizing on existing HWT hardware, nitrogen storage, vacuum capacity and diagnostics, both the cost and the time required for tunnel modifications were minimized. All wind tunnel modifications, nozzles and pitot pressure measurement hardware were designed, constructed, installed and ready to use in only four months.

Uncertainties in Mach number due to pitot pressure measurement error and instrumentation error were very small, as indicated by the excellent repeatability of the data. The average standard deviation in Mach number for 36 repeated surveys was 0.00323 . The excellent repeatability, coupled with the fact that the assumptions underlying the Rayleigh pitot probe formula are exactly met for pitot measurements in the inviscid flow field of each nozzle, suggest that the simple pitot probe was the ideal measurement technique for determining flow field Mach number in this experiment.

Probe axial position errors relative to the nozzle exit plane were estimated to be approximately \pm 0.007 inches. Because the nozzle flow is expanding rapidly, this probe axial position uncertainty produced an uncertainty in Mach number of \pm 0.007 . These probe position errors prevented us from performing a successful in situ calibration of probe angle effects using pitot probes placed at an angle to the nozzle centerline. 
Pitot pressure surveys showed that the throat geometry used in the Baseline nozzle configurations produced adequate flow uniformity and quality for the stated operational purpose of producing torque. The Mach number obtained from pitot probe surveys at the nozzle exit centerline ( $Y=0, Z=+0.010$ inches) varied from $\sim 3.5$ to 3.75 across the inviscid flow field. However, the flow fields produced by the two Baseline nozzles were not the same; although the basic wave structures were the same in each profile, the wave strengths and locations were different. Furthermore, both nozzle flow fields were slightly asymmetric. The same results were observed in the Configuration A and B nozzles, which also used the same throat geometry as the flight vehicle nozzle configuration but had a smaller throat diameter.

Although a detailed inspection of these nozzles in the machine shop showed no measurable differences in throat geometry and dimensions, the survey results indicate that such differences must exist and that they caused quantifiable flow asymmetries and differences in the flow field produced by supposedly identical nozzles. Because we cannot identify the specific throat geometric features and dimensional differences that caused the flow asymmetries and differences between paired nozzles, data from the flow fields generated by the Baseline and Configuration A and B nozzles cannot be used for Computational Fluid Dynamics code validation.

A weak (nearly isentropic) compression wave was noted in the surveys and in Schlieren photographs of each nozzle, independent of the throat geometry. We speculate that the wave is caused by the discontinuity in wall radius of curvature where the throat region meets the 15-degree nozzle expansion section. The angular momentum of the flow near the wall must suddenly go to zero at this discontinuity; the process of doing so may give rise to the observed weak compression wave.

The pitot survey data showed that changing the nozzle throat configuration from the abrupt wall slope changes used in the Baseline and Configuration A and B nozzles to the 0.500-inch streamwise radius of curvature used in the Configuration $\mathrm{C}$ and $\mathrm{D}$ nozzles made a large difference in the nozzle centerline exit flow field. The 0.500 -inch streamwise radius of curvature eliminated the problem that produced both flow asymmetries and measurable differences between the flow fields of the pairs of Baseline and Configuration $\mathrm{A}$ and $\mathrm{B}$ nozzles. It also appears to have eliminated any flow separation in the throat region. The two nozzles in each pair produced nearly identical flow fields. Therefore, we conclude that data from the Configuration $\mathrm{C}$ and $\mathrm{D}$ nozzles can be used for CFD code validation.

The inviscid flow features and Mach numbers for Configuration $\mathrm{C}$ and $\mathrm{D}$ nozzles change very little (by only a few hundredths of a Mach number) at any given flow field location as the stagnation pressure is varied from 62 to $1000 \mathrm{lbs} / \mathrm{in}^{2}$. The changes in the boundary layer displacement thickness caused by this range of nozzle stagnation pressure make small changes in the location and magnitude of the inviscid flow features, but they do not change their basic character. 


\section{References}

1. Fearn, R. and Weston, R. P., "Vorticity Associated with a Jet in a Cross Flow," AIAA Journal Vol. 12, No. 12, December 1974, pp 1666-1671.

2. Chocinski, D., Leblanc, R. and Hachemin, J. -V., "Experimental/Computational Investigation of Supersonic Jet in a Subsonic Compressible Crossflow," Paper No. 970714, AIAA 35th Aerospace Sciences Meeting, Reno, NV, January 1997.

3. Beresh, S. J., Henfling, J. F. and Erven, R. J., "Flow Separation Inside a Supersonic Nozzle Exhausting into a Subsonic Compressible Flow," J. Propulsion and Power, Vol. 19, No. 4, July-August 2003, pp. 655-662.

4. Beresh, S. J., Henfling, J. F., Erven, R. J. and Spillers, R., "Penetration of a Transverse Supersonic Jet into a Subsonic Compressible Crossflow," AIAA J. Vol. 43, No. 2, February 2005, pp. 379-389.

5. Beresh, S. J., Henfling, J. F., Erven, R. J. and Spillers, R., "Turbulent Characteristics of a Transverse Supersonic Jet in a Subsonic Compressible Crossflow, AIAA J. Vol. 43, No.11, November 2005, pp.2385-2394.

6. Beresh, S. J., Heineck, J. T., Walker, S. M., Schairer, E. T., and Yaste, D. M., "Planar Velocimetry on a Full-Scale Flight Vehicle Configuration," submitted to the AIAA Journal.

7. Beresh, S. J., Henfling, J. F., Erven, R. J. and Spillers, R., "Vortex Structure Produced by a laterally-Inclined Supersonic Jet in Transonic Crossflow," submitted to the Journal of Power \& Propulsion.

8. Beresh, S. J., Henfling, J. F., Erven, R. J. and Spillers, R. W., "Crossplane Velocimetry of a Transverse Supersonic Jet in a Transonic Crossflow," AIAA Paper 2006-0906, 44 AIAA Aerospace Sciences Meeting and Exhibit, January 9-12, 2006.

9. Maydew, R. C., "Sandia Laboratory Aerodynamic Test Facilities," Sandia Laboratories, SC-4937(M), September 1963.

10. Daum, F. L. and Gyarmathy, G., "Condensation of Air and Nitrogen in Hypersonic Wind Tunnels," AIAA J., Vol. 6, No. 3, March 1968, pp. 458-465.

11. Equations, Tables and Charts for Compressible Flow, NACA Report 1135, 1953.

12. Reed, J. F., Peterson, C. W., and Curry, W. H., "Electric Heater Development and Performance Data for A Mach 1`4 Wind Tunnel," J. Spacecraft and Rockets, Vol. 12, No. 5, May 1975, pp. 308-313.

13. Oberkampf, W. L., Aeschliman, D. P., Henfling, J. F. and Larson, D. E., "Surface Pressure Measurements for CFD Code Validation in Hypersonic Flow," Paper No. AIAA 95-2273, 26th AIAA Fluid Dynamics Conference, San Diego, CA, June 1995.

14. Aeschliman, D. P. and Oberkampf, W. L., "Experimental Methodology for Computational Fluid Dynamics Code Validation," AIAA J., Vol. 36, No. 5, May 1998, pp. 733-741.

15. Chue, S. H., "Pressure Probes for Fluid Measurement," Prog. Aerospace Sci., Vol. 16, No. 2, 1975, pp. 147-223. 
$-50-$ 


\section{Appendix:}

\section{Run Log for the Nozzle Characterization Experiments in the Hypersonic Wind Tunnel}

$\begin{array}{rc}\begin{array}{r}\text { Run } \\ \text { No. }\end{array} & \text { Nozzle } \\ 1-28 & 0 \\ 29 & 0 \\ 30 & 0 \\ 31 & 0 \\ 32 & 0 \\ 33 & 0 \\ 34 & 0 \\ 35 & 1 \\ 36 & 1 \\ 37 & 1 \\ 38 & 1 \\ 39 & 10 \\ 40 & 10 \\ 41 & 10 \\ 42 & 10 \\ 43 & 11 \\ 44 & 11 \\ 45 & 11 \\ 46 & 11 \\ 47 & 20 \\ 48 & 20 \\ 49 & 20 \\ 50 & 20 \\ 51 & 20 \\ 52 & 21 \\ 53 & 21 \\ 54 & 21 \\ 55 & 21\end{array}$

$P_{0}, \mathbf{l b s} / \mathbf{i n}^{2}$
62 to 1000
63
63
78
94
94
63
63
78
63
94
94
63
78
78
63
78
94
78
63
63
78
94
78
63
78
94
78

Nozzle Ro
Angle, de
0
0
0
0
180
0
0
0
0
0
0
0
0
180
0
0
0
180
0
0
0
0
0
0
0
0
0
0
0
0
0
0
0
0
0
0

Survey

Type Comments, Notes

axial Shakedown runs using Baseline Nozzle 0

Baseline Nozzle 1

Nozzle Configuration A-10

Nozzle Configuration A-11

Nozzle Configuration B-20

Nozzle Configuration B-21 
Run

No. Nozzle $P_{0}, \mathrm{lbs} / \mathrm{in}^{2}$

$\begin{array}{lll}56 & 0 & 63\end{array}$

$\begin{array}{lll}57 \quad 0 & 78\end{array}$

$\begin{array}{lll}58 & 0 & 94\end{array}$

$\begin{array}{lll}59 & 0 & 78\end{array}$

$\begin{array}{lll}60 & 1 & 78\end{array}$

$\begin{array}{lll}61 & 21 & 1000\end{array}$

$\begin{array}{lll}62 & 21 & 1000\end{array}$

$63 \quad 11 \quad 1000$

$\begin{array}{lll}64 & 1 & 1000\end{array}$

$65 \quad 21$

$66 \quad 11$

$67 \quad 21$

$68 \quad 21$

$69 \quad 21$

$70 \quad 21$

$71 \quad 20-2$

$72 \quad 20-2$

$73 \quad 20-2$

$74 \quad 0-2$

$75 \quad 0-2$

$76 \quad 0-2$

$77 \quad 1$

$78 \quad 1$

$79 \quad 1$

$80 \quad 11-2$

$81 \quad 11-2$

$82 \quad 11-2$

$83 \quad 10$

$84 \quad 10$

$85 \quad 10$

63

94

78

78

Nozzle Roll
Angle, deg.

Angle

0

0

0

180

180

0

0

0

0

0

$\begin{array}{cc}750 & 0 \\ 63,78,94 & 0\end{array}$

$63,78,94$

63, 78, 94

63, 78, 94

$63,78,94$

63, 78, 94

$63,78,94$

$63,78,94$

$63,78,94$

63, 78, 94

$63,78,94$

63, 78, 94

63, 78, 94

63, 78, 94

63, 78, 94

63, 78, 94

63, 78, 94

63, 78, 94

63, 78, 94
Survey

Type

axial

Comments, Notes

Nozzle 20 was polished, designated B-20-2

Nozzle 0 was polished, designated Baseline 0-2

Nozzle 11 was polished, designated A-11-2 


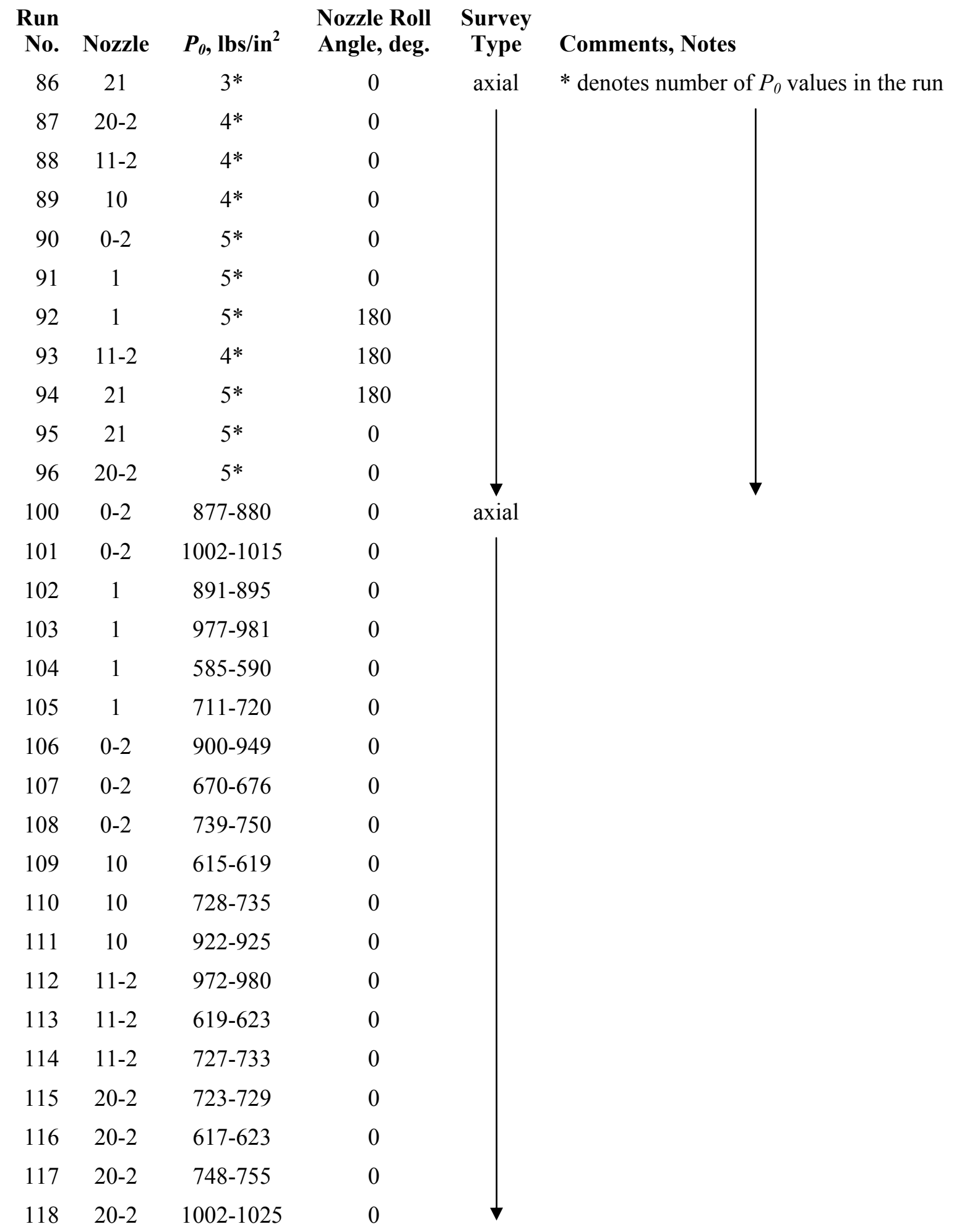




\begin{tabular}{|c|c|c|c|c|c|}
\hline $\begin{array}{c}\text { Run } \\
\text { No. }\end{array}$ & Nozzle & $P_{0}, \operatorname{lbs} / \mathbf{i n}^{2}$ & $\begin{array}{l}\text { Nozzle Roll } \\
\text { Angle, deg. }\end{array}$ & $\begin{array}{c}\text { Survey } \\
\text { Type }\end{array}$ & Comments, Notes \\
\hline 119 & 21 & $624-628$ & 0 & axial & \\
\hline 120 & 21 & $747-752$ & 0 & & \\
\hline 121 & 21 & 989-1004 & 0 & & \\
\hline 122 & $0-2$ & $620-626$ & 0 & & \\
\hline 123 & 1 & $619-622$ & 0 & & \\
\hline 124 & 1 & 784-791 & 0 & & \\
\hline 125 & 1 & $619-623$ & 180 & & \\
\hline 126 & 10 & $946-948$ & 0 & & \\
\hline 127 & 10 & $312 \& 375$ & 0 & & \\
\hline 128 & 10 & $250,312,375$ & 0 & & \\
\hline 129 & $11-2$ & $250,312,375$ & 0 & & \\
\hline 130 & $21-2$ & $312 \& 375$ & 0 & & \\
\hline 131 & $20-2$ & $312 \& 375$ & 0 & & \\
\hline 132 & $20-2$ & $437 \& 500$ & 0 & & \\
\hline 133 & $0-2$ & $312 \& 375$ & 0 & & \\
\hline 134 & $0-2$ & $437 \& 500$ & 0 & & Nozzle B-21 was polished after this run \\
\hline 135 & 20 & $312 \& 375$ & 0 & & \\
\hline 136 & $21-2$ & $312 \& 375$ & 0 & & Nozzle 21 now designated B-21-2 \\
\hline 137 & $21-2$ & $437 \& 500$ & 0 & & \\
\hline 138 & 1 & $312 \& 375$ & 0 & & \\
\hline 139 & 1 & $437 \& 500$ & 0 & & \\
\hline 140 & 10 & $437 \& 500$ & 0 & & \\
\hline 141 & $11-2$ & $437 \& 500$ & 0 & & \\
\hline 142 & $20-2$ & $437 \& 500$ & 0 & & \\
\hline 143 & $20-2$ & 850 & 0 & & \\
\hline 144 & 1 & 800 & 0 & & \\
\hline 145 & $0-2$ & 800 & 0 & & \\
\hline 146 & 10 & 900 & 0 & & \\
\hline 147 & 10 & 800 & 0 & & \\
\hline 148 & $11-2$ & 900 & 0 & & \\
\hline 149 & $11-2$ & 800 & 0 & $\downarrow$ & \\
\hline
\end{tabular}




$\begin{array}{rccccl}\begin{array}{r}\text { Run } \\ \text { No. }\end{array} & \text { Nozzle } & \boldsymbol{P}_{\mathbf{0}}, \mathbf{l b s} / \text { in }^{2} & \begin{array}{c}\text { Nozzle Roll } \\ \text { Angle, deg. }\end{array} & \begin{array}{c}\text { Survey } \\ \text { Type }\end{array} & \text { Comments, Notes } \\ 150 & 21-2 & 750 & 0 & \text { axial } \\ 151 & 21-2 & 850 & 0 & \mid \\ 152 & 21-2 & 950 & 0 & \\ 153 & 21-2 & 1000 & 0 & \\ 154 & 21-2 & 800 & 0 & \\ 155 & 21-2 & 625 & 0 & * P_{0}=125,156,188,219 \& 250 \mathrm{lbs} / \mathrm{in}^{2} \\ 156 & 21-2 & 5 * & 0 & * P_{0}=63,78 \& 94 \mathrm{lbs} / \mathrm{in}^{2} \\ 157 & 21-2 & 3 * & 0 & \downarrow\end{array}$

Runs 30-157 were axial surveys on the nozzle centerline. Run 160 begins lateral surveys at $Y=0$.

\begin{tabular}{lllll}
160 & $0-2$ & 94 & 0 & \multicolumn{1}{c}{ lateral } \\
161 & $0-2$ & 63 & 0 \\
162 & $0-2$ & 63 & 0 \\
163 & $0-2$ & 63 & 0 \\
164 & $0-2$ & 63 & 0 \\
165 & $0-2$ & 63 & 0 \\
167 & $0-2$ & 78 & 0 \\
168 & $0-2$ & 125 & 0 \\
169 & $0-2$ & 188 & 0 \\
170 & $0-2$ & 250 & 0 \\
172 & $0-2$ & 375 & 0 \\
173 & $0-2$ & 375 & 0 \\
174 & $0-2$ & 500 & 0 \\
175 & $0-2$ & 625 & 0 \\
176 & $0-2$ & 770 & 0 \\
177 & $0-2$ & 930 & 0 \\
178 & $20-2$ & 860 & 0 \\
179 & $20-2$ & 63 & 0 \\
180 & $20-2$ & 78 & 0 \\
181 & $20-2$ & 63 & 94 \\
182 & $20-2$ & 94 & \\
183 & $20-2$ & 94 &
\end{tabular}




\begin{tabular}{|c|c|c|c|c|c|}
\hline $\begin{array}{c}\text { Run } \\
\text { No. }\end{array}$ & Nozzle & $P_{0}, \mathrm{lbs} / \mathrm{in}^{2}$ & $\begin{array}{l}\text { Nozzle Roll } \\
\text { Angle, deg. }\end{array}$ & $\begin{array}{c}\text { Survey } \\
\text { Type }\end{array}$ & Comments, Notes \\
\hline 184 & $20-2$ & 1000 & 0 & lateral & \\
\hline 185 & $20-2$ & 750 & 0 & & \\
\hline 188 & $20-2$ & 750 & 0 & & \\
\hline 189 & $20-2$ & 500 & 0 & & \\
\hline 190 & $20-2$ & 437 & 0 & & \\
\hline 191 & $20-2$ & 375 & 0 & & \\
\hline 192 & $20-2$ & 125 & 0 & & \\
\hline 193 & $20-2$ & 188 & 0 & & \\
\hline 194 & $20-2$ & 250 & 0 & & \\
\hline 195 & $20-2$ & 250 & 0 & & \\
\hline 196 & $20-2$ & 188 & 0 & & \\
\hline 197 & $21-2$ & 750 & 0 & & \\
\hline 198 & $21-2$ & 800 & 0 & & \\
\hline 199 & $21-2$ & 900 & 0 & & \\
\hline 200 & $21-2$ & 680 & 0 & & \\
\hline 201 & $21-2$ & 625 & 0 & & \\
\hline 202 & $21-2$ & 1000 & 0 & & \\
\hline 203 & $21-2$ & 375 & 0 & & \\
\hline 204 & $21-2$ & 437 & 0 & & \\
\hline 205 & $21-2$ & 375 & 0 & & \\
\hline 206 & $21-2$ & 500 & 0 & & \\
\hline 207 & $21-2$ & 125 & 0 & & \\
\hline 208 & $21-2$ & 188 & 0 & & \\
\hline 209 & $21-2$ & 250 & 0 & & \\
\hline 210 & $21-2$ & 63 & 0 & & \\
\hline 211 & $21-2$ & 78 & 0 & & \\
\hline 212 & $21-2$ & 94 & 0 & & \\
\hline 213 & $21-2$ & 63 & 0 & & \\
\hline 214 & 10 & 63 & 0 & & \\
\hline 215 & 10 & 78 & 0 & & \\
\hline 216 & 10 & 94 & 0 & & \\
\hline 217 & 10 & 94 & 0 & & \\
\hline 218 & 10 & 750 & 0 & $\downarrow$ & \\
\hline
\end{tabular}




\begin{tabular}{|c|c|c|c|c|c|}
\hline $\begin{array}{c}\text { Run } \\
\text { No. }\end{array}$ & Nozzle & $P_{0}, \mathrm{lbs} / \mathrm{in}^{2}$ & $\begin{array}{l}\text { Nozzle Roll } \\
\text { Angle, deg. }\end{array}$ & $\begin{array}{c}\text { Survey } \\
\text { Type }\end{array}$ & Comments, Notes \\
\hline 219 & 10 & 620 & 0 & lateral & \\
\hline 220 & 10 & 1000 & 0 & & \\
\hline 221 & 10 & 940 & 0 & & \\
\hline 222 & 10 & 930 & 0 & & Reverse sweep \\
\hline 223 & 10 & 625 & 0 & & \\
\hline 224 & 10 & 500 & 0 & & \\
\hline 225 & 10 & 437 & 0 & & \\
\hline 226 & 10 & 312 & 0 & & \\
\hline 227 & 10 & 250 & 0 & & \\
\hline 228 & 10 & 125 & 0 & & \\
\hline 229 & 10 & 188 & 0 & & \\
\hline 230 & 10 & 250 & 0 & & \\
\hline 231 & $11-2$ & 125 & 0 & & \\
\hline 232 & $11-2$ & 188 & 0 & & \\
\hline 233 & $11-2$ & 250 & 0 & & \\
\hline 234 & $11-2$ & 250 & 0 & & \\
\hline 235 & $11-2$ & 730 & 0 & & \\
\hline 236 & $11-2$ & 625 & 0 & & \\
\hline 237 & $11-2$ & 970 & 0 & & \\
\hline 238 & $11-2$ & 770 & 0 & & \\
\hline 239 & $11-2$ & 830 & 0 & & \\
\hline 240 & $11-2$ & 312 & 0 & & \\
\hline 241 & $11-2$ & 437 & 0 & & \\
\hline 242 & $11-2$ & 500 & 0 & & \\
\hline 243 & $11-2$ & 63 & 0 & & \\
\hline 244 & $11-2$ & 78 & 0 & & \\
\hline 245 & $11-2$ & 94 & 0 & & \\
\hline 246 & $11-2$ & 94 & 0 & & \\
\hline 247 & 1 & 625 & 0 & & \\
\hline 248 & 1 & 820 & 0 & & \\
\hline 249 & 1 & 870 & 0 & & \\
\hline 250 & 1 & 900 & 0 & & \\
\hline 251 & 1 & 1000 & 0 & $\downarrow$ & Close to limit on $P_{0}$ transducer \\
\hline
\end{tabular}




\begin{tabular}{|c|c|c|c|c|c|}
\hline $\begin{array}{c}\text { Run } \\
\text { No. }\end{array}$ & Nozzle & $P_{0}, \mathrm{lbs} / \mathrm{in}^{2}$ & $\begin{array}{l}\text { Nozzle Roll } \\
\text { Angle, deg. }\end{array}$ & $\begin{array}{c}\text { Survey } \\
\text { Type }\end{array}$ & Comments, Notes \\
\hline 252 & 1 & 750 & 0 & lateral & \\
\hline 253 & 1 & 1000 & 0 & & \\
\hline 254 & 1 & 312 & 0 & & \\
\hline 255 & 1 & 375 & 0 & & \\
\hline 256 & 1 & 500 & 0 & & \\
\hline 257 & 1 & 375 & 0 & & \\
\hline 258 & 1 & 125 & 0 & & Used higher pressure range transducers \\
\hline 259 & 1 & 500 & 0 & & Started sweep with $Z=0.76$ inches \\
\hline 260 & 1 & 250 & 0 & & \\
\hline 261 & 1 & 188 & 0 & & \\
\hline 262 & 1 & 125 & 0 & & \\
\hline 263 & 1 & 63 & 0 & & \\
\hline 264 & 1 & 78 & 0 & & \\
\hline 265 & 1 & 94 & 0 & & \\
\hline 266 & 30 & 63 & 0 & & Nozzle Configuration D-30 \\
\hline 267 & 30 & 78 & 0 & & \\
\hline 268 & 30 & 94 & 0 & & \\
\hline 269 & 30 & 125 & 0 & & \\
\hline 270 & 30 & 188 & 0 & & \\
\hline 271 & 30 & 250 & 0 & & \\
\hline 272 & 30 & 250 & 0 & & \\
\hline 273 & 30 & 625 & 0 & & \\
\hline 274 & 30 & 750 & 0 & & \\
\hline 275 & 30 & 990 & 0 & & \\
\hline 276 & 30 & 820 & 0 & & \\
\hline 277 & 30 & 880 & 0 & & \\
\hline 278 & 30 & 820 & 0 & & \\
\hline 279 & 30 & 500 & 0 & & \\
\hline 280 & 30 & 312 & 0 & & \\
\hline 281 & 30 & 437 & 0 & & \\
\hline 282 & 30 & 490 & 0 & $\downarrow$ & \\
\hline
\end{tabular}




\begin{tabular}{|c|c|c|c|c|c|}
\hline $\begin{array}{r}\text { Run } \\
\text { No. }\end{array}$ & Nozzle & $P_{0}$, lbs/in ${ }^{2}$ & $\begin{array}{l}\text { Nozzle Roll } \\
\text { Angle, deg. }\end{array}$ & $\begin{array}{c}\text { Survey } \\
\text { Type }\end{array}$ & Comments, Notes \\
\hline 283 & 40 & 500 & 0 & lateral & Nozzle Configuration C-40 \\
\hline 284 & 40 & 850 & 0 & & \\
\hline 285 & 40 & 930 & 0 & & \\
\hline 286 & 40 & 960 & 0 & & \\
\hline 287 & 40 & 63 & 0 & & \\
\hline 288 & 40 & 125 & 0 & & \\
\hline 289 & 40 & 250 & 0 & & \\
\hline 290 & 41 & 250 & 0 & & Nozzle Configuration C-41 \\
\hline 291 & 41 & 125 & 0 & & \\
\hline 292 & 41 & 63 & 0 & & \\
\hline 293 & 41 & 63 & 0 & & \\
\hline 294 & 41 & 950 & 0 & & \\
\hline 295 & 41 & 480 & 0 & & \\
\hline 296 & 31 & 500 & 0 & & Nozzle Configuration D-31 \\
\hline 297 & 31 & 63 & 0 & & \\
\hline 298 & 31 & 125 & 0 & & \\
\hline 299 & 31 & 250 & 0 & & \\
\hline 300 & 31 & 1000 & 0 & & \\
\hline 301 & 31 & 700 & 0 & & \\
\hline 302 & 31 & 500 & 90 & & \\
\hline 303 & $21-2$ & 500 & 90 & $\downarrow$ & \\
\hline
\end{tabular}

All surveys made for Runs 1-303 are for a single pitot probe at zero degrees angle of attack relative to the nozzle centerline. For Runs 304-319, the pitot probe was set at a +5 -degree angle of attack relative to the nozzle centerline. "Plus 5 degrees" means that the probe tip was angled 5 degrees to the west in the HWT.

$\begin{array}{lllll}304 & 30 & 500 & 0 & \text { lateral } \\ 305 & 30 & 500 & 0 & \\ 306 & 30 & 720 & 0 & \\ 307 & 30 & 980 & 0 \\ 308 & 30 & 250 & 0 \\ 309 & 30 & 125 & 0 & \downarrow \\ 310 & 30 & 63 & 0 & \end{array}$




$\begin{array}{cccc}\begin{array}{r}\text { Run } \\ \text { No. }\end{array} & \text { Nozzle } & \boldsymbol{P}_{\mathbf{0}}, \mathbf{l b s} / \mathbf{i n}^{2} & \begin{array}{c}\text { Nozzle Role, } \\ \text { Angle, }\end{array} \\ 311 & 40 & 63 & 0 \\ 312 & 40 & 840 & 0 \\ 313 & 40 & 1000 & 0 \\ 314 & 40 & 1000 & 0 \\ 315 & 40 & 125 & 0 \\ 316 & 40 & 250 & 0 \\ 317 & 40 & 500 & 0 \\ 318 & 40 & 930 & 0 \\ \text { Runs } 319-323 \text { are } 3-\text { probe checkout runs. } \\ 319 & 40 & 250 & 0 \\ 320 & 40 & 250 & 0 \\ 321 & 40 & 375 & 0 \\ 322 & 40 & 250 & 0 \\ 323 & 40 & 250 & 0\end{array}$

Runs 329-428 are 3-probe surveys. For Runs $329-378$, center probe is on nozzle centerline.

\begin{tabular}{cccc|c}
329 & 1 & 500 & 0 & $12 / 4 / 2002$ \\
330 & 1 & 400 & 0 & $12 / 5 / 2002$ \\
331 & 1 & 63 & 0 & \\
332 & 1 & 100 & 0 & \\
333 & 1 & 155 & 0 & \\
334 & 1 & 150 & 0 & \\
335 & $0-2$ & 63 & 0 & \\
336 & $0-2$ & 100 & 0 & \\
337 & $0-2$ & 150 & 0 & \\
338 & $0-2$ & 400 & 0 & \\
339 & $0-2$ & 500 & 0 & \\
340 & $0-2$ & 500 & 0 & \\
341 & 40 & 500 & 0 & \\
342 & 40 & 400 & 0 & \\
343 & 40 & 63 & 0 & \\
344 & 40 & 100 & 0 & \\
345 & 40 & 150 & 0 &
\end{tabular}




\begin{tabular}{|c|c|c|c|c|c|}
\hline $\begin{array}{c}\text { Run } \\
\text { No. }\end{array}$ & Nozzle & $P_{0}, \mathrm{lbs} / \mathrm{in}^{2}$ & $\begin{array}{l}\text { Nozzle Roll } \\
\text { Angle, deg. }\end{array}$ & $\begin{array}{c}\text { Survey } \\
\text { Type }\end{array}$ & Comments, Notes \\
\hline 347 & 30 & 63 & 0 & lateral & \\
\hline 348 & 30 & 100 & 0 & & \\
\hline 349 & 30 & 150 & 0 & & \\
\hline 350 & 30 & 400 & 0 & & \\
\hline 351 & 30 & 500 & 0 & & \\
\hline 352 & $20-2$ & 400 & 0 & & \\
\hline 353 & $20-2$ & 500 & 0 & & \\
\hline 354 & $20-2$ & 65 & 0 & & \\
\hline 355 & $20-2$ & 100 & 0 & & \\
\hline 356 & $20-2$ & 150 & 0 & & \\
\hline 357 & $21-2$ & 70 & 0 & & \\
\hline 358 & $21-2$ & 100 & 0 & & \\
\hline 359 & $21-2$ & 150 & 0 & & \\
\hline 360 & $21-2$ & 400 & 0 & & \\
\hline 361 & $21-2$ & 500 & 0 & & \\
\hline 362 & $11-2$ & 70 & 0 & & \\
\hline 363 & $11-2$ & 100 & 0 & & \\
\hline 364 & $11-2$ & 150 & 0 & & \\
\hline 365 & $11-2$ & 400 & 0 & & \\
\hline 366 & $11-2$ & 500 & 0 & & \\
\hline 367 & 10 & 400 & 0 & & \\
\hline 368 & 10 & 500 & 0 & & \\
\hline 369 & 10 & 400 & 0 & & \\
\hline 370 & 10 & 70 & 0 & & \\
\hline 371 & 10 & 100 & 0 & & \\
\hline 372 & 10 & 150 & 0 & & \\
\hline 373 & 40 & 100 & 0 & & $1 / 7 / 2003$ \\
\hline 374 & 40 & 150 & 0 & & 2/12/03; Nozzle block for alignment; position 1 \\
\hline 375 & 40 & 150 & 0 & & \\
\hline 376 & 40 & 100 & 0 & & \\
\hline 377 & 40 & 400 & 0 & & \\
\hline 378 & 40 & 500 & 0 & $\downarrow$ & \\
\hline
\end{tabular}




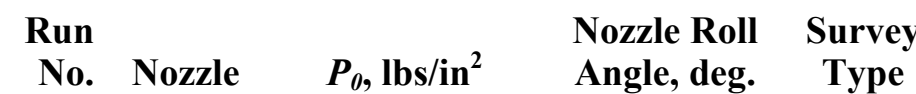

For Runs 379-410, center probe is 0.25 inches lateral above nozzle centerline.

\begin{tabular}{|c|c|c|c|c|}
\hline 379 & 40 & 400 & 0 & $2 / 17 / 2003$ \\
\hline 380 & 40 & 500 & 0 & \\
\hline 381 & 40 & 100 & 0 & \\
\hline 382 & 40 & 150 & 0 & \\
\hline 383 & 40 & 100 & 0 & \\
\hline 384 & 40 & 150 & 0 & \\
\hline 385 & 40 & 400 & 0 & \\
\hline 386 & 40 & 500 & 0 & \\
\hline 387 & $0-2$ & 400 & 0 & $2 / 18 / 2003$ \\
\hline 388 & $0-2$ & 500 & 0 & \\
\hline 389 & $0-2$ & 100 & 0 & \\
\hline 390 & $0-2$ & 150 & 0 & \\
\hline 391 & 1 & 100 & 0 & \\
\hline 392 & 1 & 150 & 0 & \\
\hline 393 & 1 & 400 & 0 & \\
\hline 394 & 1 & 500 & 0 & \\
\hline 395 & $20-2$ & 400 & 0 & \\
\hline 396 & $20-2$ & 500 & 0 & \\
\hline 397 & $20-2$ & 100 & 0 & \\
\hline 398 & $20-2$ & 150 & 0 & \\
\hline 399 & $21-2$ & 100 & 0 & \\
\hline 400 & $21-2$ & 150 & 0 & \\
\hline 401 & $21-2$ & 400 & 0 & \\
\hline 402 & $21-2$ & 500 & 0 & \\
\hline 403 & 10 & 400 & 0 & $2 / 19 / 2003$ \\
\hline 404 & 10 & 500 & 0 & \\
\hline 405 & 10 & 100 & 0 & \\
\hline 406 & 10 & 150 & 0 & \\
\hline 407 & $11-2$ & 100 & 0 & \\
\hline 408 & $11-2$ & 150 & 0 & \\
\hline 409 & $11-2$ & 400 & 0 & \\
\hline 410 & $11-2$ & 500 & 0 & \\
\hline
\end{tabular}




\section{Run Nozzle Roll Survey \\ No. Nozzle $P_{0}$, lbs/in $^{2} \quad$ Angle, deg. Type Comments, Notes}

For Runs 411-422, center probe is 0.125 inches lateral above nozzle centerline.

$\begin{array}{llll}411 & 40 & 400 & 0 \\ 412 & 40 & 500 & 0 \\ 413 & 30 & 400 & 0 \\ 414 & 30 & 500 & 0 \\ 415 & 0-2 & 400 & 0 \\ 416 & 0-2 & 500 & 0 \\ 417 & 0-2 & 100 & 0 \\ 418 & 0-2 & 150 & 0 \\ 419 & 30 & 100 & 0 \\ 420 & 30 & 150 & 0 \\ 421 & 40 & 100 & 0 \\ 422 & 40 & 150 & 0\end{array}$

For Runs $423-428$, center probe is 0.375 inches above nozzle centerline.

$\begin{array}{llll}423 & 40 & 100 & 0 \\ 424 & 40 & 150 & 0 \\ 425 & 30 & 100 & 0 \\ 426 & 30 & 150 & 0 \\ 427 & 30 & 400 & 0 \\ 428 & 30 & 500 & 0\end{array}$

Runs $429-442$ use a single probe at +5 degrees

\begin{tabular}{llll|l}
429 & 40 & 400 & 0 & $4 / 10 / 2003$ \\
430 & 40 & 450 & 0 & \\
431 & 40 & 470 & 0 & $4 / 11 / 2003$ \\
432 & 40 & 490 & 0 & \\
433 & 40 & 100 & 0 & \\
434 & 40 & 150 & 0 & \\
435 & 30 & 100 & 0 & \\
436 & 30 & 150 & 0 & \\
437 & 30 & 400 & 0 & \\
438 & 30 & 500 & 0 &
\end{tabular}




\begin{tabular}{|c|c|c|c|c|c|}
\hline $\begin{array}{c}\text { Run } \\
\text { No. }\end{array}$ & Nozzle & $P_{0}, \mathrm{lbs} / \mathrm{in}^{2}$ & $\begin{array}{l}\text { Nozzle Roll } \\
\text { Angle, deg. }\end{array}$ & $\begin{array}{c}\text { Survey } \\
\text { Type }\end{array}$ & Comments, Notes \\
\hline 440 & $0-2$ & 500 & 0 & lateral & \\
\hline 441 & $0-2$ & 100 & 0 & & \\
\hline 442 & $0-2$ & 150 & 0 & & \\
\hline $\begin{array}{l}\text { Runs } \\
\text { (probe }\end{array}$ & $\begin{array}{l}43-454 \\
\text { tip is ang }\end{array}$ & $\begin{array}{l}\text { a single pro } \\
5 \text { degrees to }\end{array}$ & $\begin{array}{l}-5 \text { degrees } \\
\text { east) }\end{array}$ & & \\
\hline 443 & 40 & 100 & 0 & & $4 / 15 / 2003$ \\
\hline 444 & 40 & 150 & 0 & & \\
\hline 445 & 40 & 400 & 0 & & \\
\hline 446 & 40 & 500 & 0 & & \\
\hline 447 & 30 & 400 & 0 & & \\
\hline 448 & 30 & 500 & 0 & & \\
\hline 449 & 30 & 100 & 0 & & \\
\hline 450 & 30 & 150 & 0 & & \\
\hline 451 & $0-2$ & 100 & 0 & & \\
\hline 452 & $0-2$ & 150 & 0 & & \\
\hline 453 & $0-2$ & 400 & 0 & & \\
\hline 454 & $0-2$ & 500 & 0 & & \\
\hline Runs & $129-442 \mathrm{u}$ & single prob & +10 degrees & & \\
\hline 455 & 40 & 400 & 0 & & $4 / 16 / 2003$ \\
\hline 456 & 40 & 500 & 0 & & \\
\hline 457 & 40 & 100 & 0 & & \\
\hline 458 & 40 & 150 & 0 & & \\
\hline 459 & 30 & 100 & 0 & & \\
\hline 460 & 30 & 150 & 0 & & \\
\hline 461 & 30 & 400 & 0 & & \\
\hline 462 & 30 & 500 & 0 & & \\
\hline 463 & $0-2$ & 400 & 0 & & \\
\hline 464 & $0-2$ & 500 & 0 & & \\
\hline 465 & $0-2$ & 100 & 0 & & \\
\hline 466 & $0-2$ & 150 & 0 & $\downarrow$ & \\
\hline
\end{tabular}




\section{Distribution}

\section{Internal}

$\begin{array}{llll}5 & \text { MS0825 } & 0003 & \text { C. W. Peterson } \\ 1 & \text { MS1106 } & 1342 & \text { R. J. Erven } \\ 1 & \text { MS0384 } & 1500 & \text { A. C. Ratzel } \\ 1 & \text { MS0825 } & 1510 & \text { W. L. Hermina } \\ 1 & \text { MS0834 } & 1512 & \text { R. D. M. Tachau } \\ 1 & \text { MS0834 } & 1512 & \text { S. J. Beresh } \\ 1 & \text { MS0834 } & 1512 & \text { J. F. Henfling } \\ 1 & \text { MS0825 } & 1515 & \text { B. Hassan } \\ 1 & \text { MS0825 } & 1515 & \text { J. L. Payne } \\ 1 & \text { MS0825 } & 1515 & \text { W. P. Wolfe } \\ 1 & \text { MS0828 } & 1533 & \text { M. A. Pilch } \\ & & & \text { Attn: W. L. Oberkampf } \\ 1 & \text { MS0885 } & 1800 & \text { J. E. Johannes } \\ 1 & \text { MS0429 } & 2100 & \text { B. C. Walker } \\ 1 & \text { MS0453 } & 2110 & \text { L. S. Walker } \\ 1 & \text { MS0447 } & 2111 & \text { J. D. Mangum } \\ 1 & \text { MS0447 } & 2111 & \text { P. R. Hooper } \\ 1 & \text { MS0447 } & 2111 & \text { P. D. Hoover } \\ 1 & \text { MS0464 } & 2997 & \text { D. F. Hodge } \\ 1 & \text { MS1158 } & 5424 & \text { K. V. Chavez } \\ 2 & \text { MS0899 } & 4536 & \text { Technical Library } \\ 2 & \text { MS9018 } & 8944 & \text { Center Technical Files }\end{array}$

